\title{
PIE on Safety-Tested Loose Particles from AGR-1 Compact 4-4-2
}

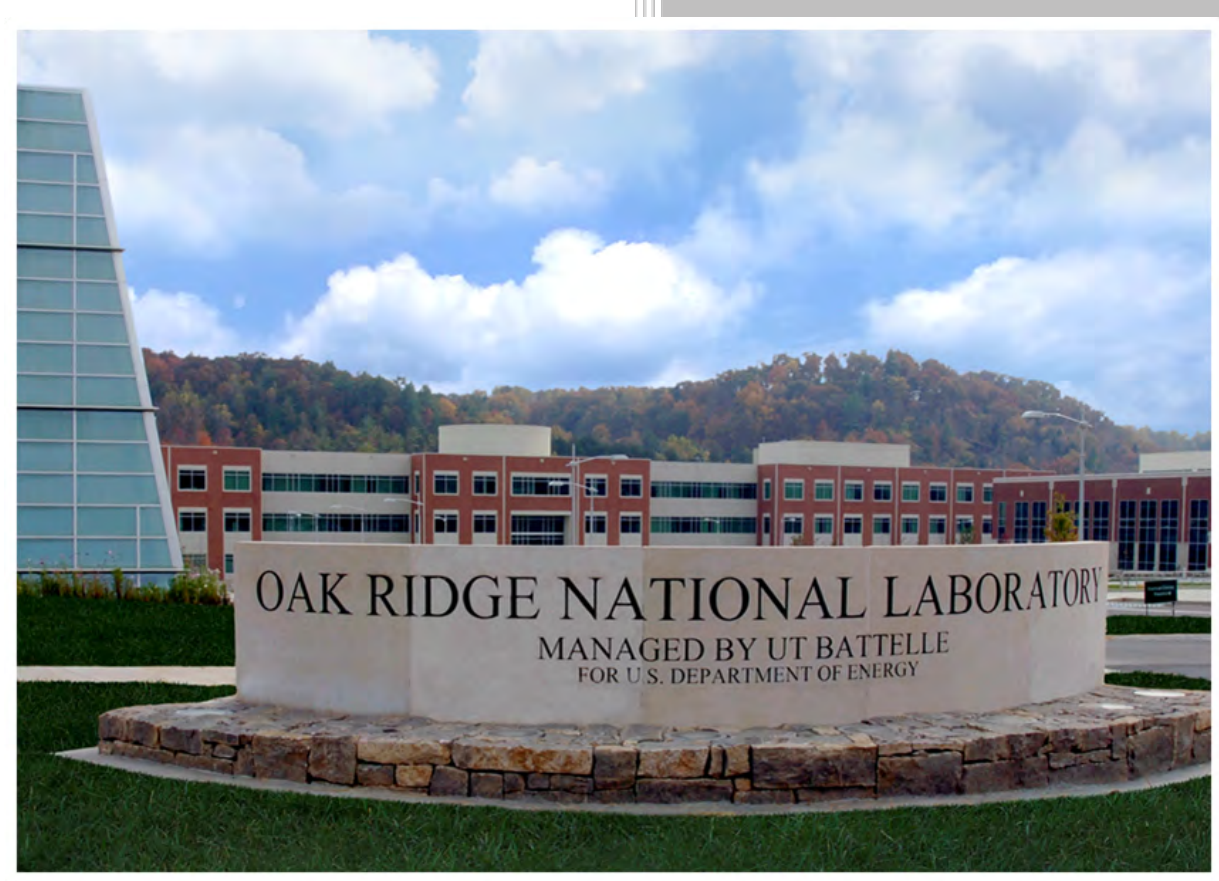

Approved for public release. Distribution is unlimited.

John D. Hunn

Tyler J. Gerczak

Robert N. Morris

Charles A. Baldwin

Fred C. Montgomery

April 2016 


\title{
DOCUMENT AVAILABILITY
}

Reports produced after January 1, 1996, are generally available free via US Department of Energy (DOE) SciTech Connect.

Website http://www.osti.gov/scitech/

Reports produced before January 1, 1996, may be purchased by members of the public from the following source:

\author{
National Technical Information Service \\ 5285 Port Royal Road \\ Springfield, VA 22161 \\ Telephone 703-605-6000 (1-800-553-6847) \\ TDD 703-487-4639 \\ Fax 703-605-6900 \\ E-mail info@ntis.gov \\ Website http://www.ntis.gov/help/ordermethods.aspx
}

Reports are available to DOE employees, DOE contractors, Energy Technology Data Exchange representatives, and International Nuclear Information System representatives from the following source:

Office of Scientific and Technical Information

PO Box 62

Oak Ridge, TN 37831

Telephone 865-576-8401

Fax 865-576-5728

E-mail reports@osti.gov

Website http://www.osti.gov/contact.html

This report was prepared as an account of work sponsored by an agency of the United States Government. Neither the United States Government nor any agency thereof, nor any of their employees, makes any warranty, express or implied, or assumes any legal liability or responsibility for the accuracy, completeness, or usefulness of any information, apparatus, product, or process disclosed, or represents that its use would not infringe privately owned rights. Reference herein to any specific commercial product, process, or service by trade name, trademark, manufacturer, or otherwise, does not necessarily constitute or imply its endorsement, recommendation, or favoring by the United States Government or any agency thereof. The views and opinions of authors expressed herein do not necessarily state or reflect those of the United States Government or any agency thereof. 
Fusion and Materials for Nuclear Systems Division

\title{
PIE ON SAFETY-TESTED LOOSE PARTICLES FROM AGR-1 COMPACT 4-4-2
}

\author{
John D. Hunn \\ Tyler J. Gerczak \\ Robert N. Morris \\ Charles A. Baldwin \\ Fred C. Montgomery
}

Date Published: April 2016

\author{
Work sponsored by \\ US DEPARTMENT OF ENERGY \\ Office of Nuclear Energy - Advanced Reactor Technologies \\ under the
}

Advanced Gas Reactor Fuel Development and Qualification Program

Prepared by OAK RIDGE NATIONAL LABORATORY

Oak Ridge, TN 37831-6283

managed by

UT-BATTELLE, LLC

for the

US DEPARTMENT OF ENERGY

under contract DE-AC05-00OR22725 



\section{CONTENTS}

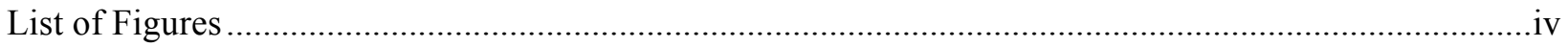

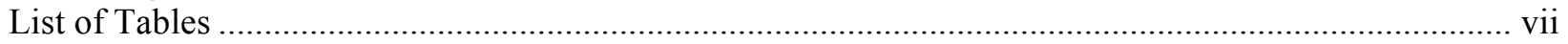

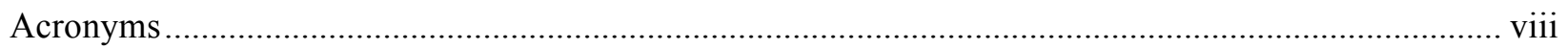

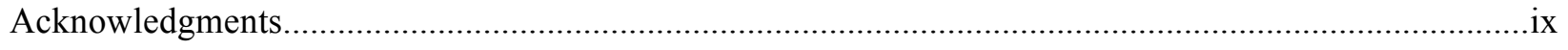

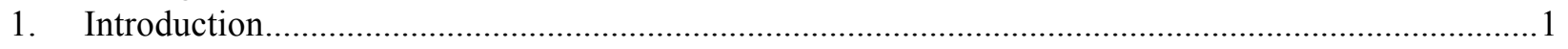

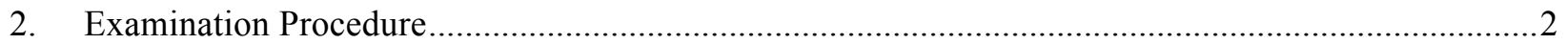

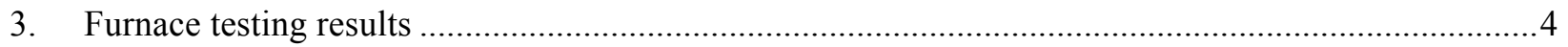

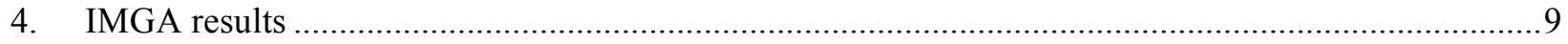

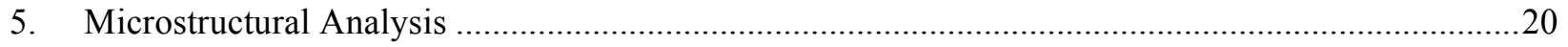

5.1 Particles with Failed TRISO - X-ray Tomography and Optical Microscopy .................20

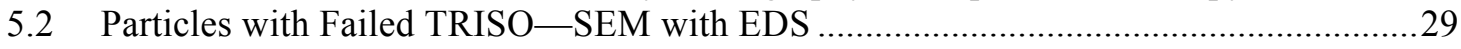

5.3 Particles that Retained Cesium — Optical Microscopy and SEM with EDS ....................39

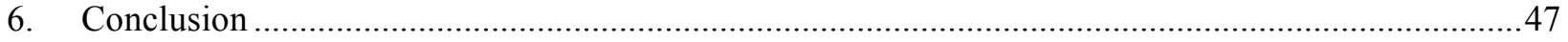

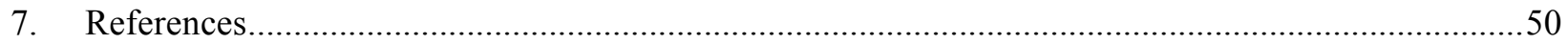




\section{LIST OF FIGURES}

1. CCCTF fuel holder assembly for the loose-particle test: a) fifteen-particle tray, b) five-tray assembly, and c) tray assembly in standard CCCTF graphite holder.

2. Cumulative release of cesium and krypton from Compact 4-4-2 loose particles at $1800^{\circ} \mathrm{C}$. Note that the magnitude of the cesium release may be off due to uncertainty in the deposition cup collection efficiency, as discussed at the end of this section.

3. Average rate of cesium release during each deposition cup collection interval...

4. Cumulative release of silver, europium, and strontium from Compact 4-4-2 loose particles at $1800^{\circ} \mathrm{C}$. Note that the magnitude of the plotted release may be off due to uncertainty in the deposition cup collection efficiency, as discussed at the end of this section.

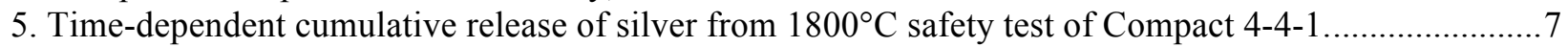

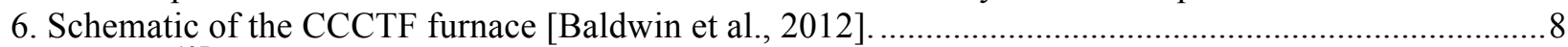

7. Ratio of ${ }^{137} \mathrm{Cs}$ in 4001 particles deconsolidated from as-irradiated Compact 4-4-2 versus the calculated inventory, adjusted for variation in fissionable material and burnup with the measured ${ }^{144}$ Ce activity [Hunn et al. 2013a, 23].

8. Ratio of ${ }^{110 \mathrm{~m}} \mathrm{Ag}$ in 90 as-irradiated Compact 4-4-2 particles versus calculated inventory, adjusted for variation in fissionable material and burnup with the measured ${ }^{137} \mathrm{Cs}$ activity [Hunn et al. 2013a, 25].

9. Ratio of ${ }^{154} \mathrm{Eu}$ in 90 as-irradiated Compact 4-4-2 particles versus calculated inventory, adjusted for variation in fissionable material and burnup with the measured ${ }^{137} \mathrm{Cs}$ activity [Hunn et al. 2013a, 24].

10. Radioisotope retention fraction of 75 particles after $1800^{\circ} \mathrm{C}$ safety testing.

11. Particle 442-A009: ( $a$ and b) oblique orthogonal x-ray tomographs showing SiC crack aligned with buffer/IPyC crack, with adjacent U-dispersion in IPyC; (c) optical micrograph of a polished section showing $\mathrm{U}$ dispersion in the IPyC; (d) another area in the same polished section where typical local $\mathrm{SiC}$ degradation at the tip of an IPyC crack was observed that was not associated with the TRISO failure.

12. Particle 442-A064: (a and b) oblique orthogonal x-ray tomographs showing SiC crack at top and bottom of image and noticeable loss of kernel material; (c) 3D visualization using threshold filter to display $\mathrm{SiC}$ surface (OPyC layer is semitransparent) to show extended $\mathrm{SiC}$ crack that almost circumnavigated this particle, $(\mathrm{d}-\mathrm{f})$ optical micrographs of polished section showing $\mathrm{SiC}$ crack and degradation at $\mathrm{IPyC} / \mathrm{SiC}$ interface.

13. Particle 442-A053: (a and b) oblique orthogonal x-ray tomographs showing through-layer SiC degradation aligned along an IPyC crack and significant uranium dispersion throughout the buffer and IPyC, (c) sectioned 3D visualization using a threshold filter to emphasize SiC and higher-density material (pyrocarbon not visible) and showing an extended $\mathrm{SiC}$ crack with degradation, ( $\mathrm{d}$ and e) optical micrographs of polished section showing metallic clusters in $\mathrm{SiC}$ and degradation extending out into $\mathrm{SiC}$ along the crack.

14. Particle 442-A053: Set of images obtained further from midplane compared to Figure 13; (a) x-ray tomograph showing same plane as (b), (b-e) optical micrograph of polished section showing SiC degradation along crack and missing pockets of IPyC associated with radial dispersions of uranium visible in (a), (f) $\mathrm{SiC}$ degradation in another plane that was parallel and close to (c) showing a crack through the degraded region.....

15. Particle 442-A072: ( $a$ and b) oblique orthogonal x-ray tomographs showing SiC crack (lower left) and kernel protrusion with significant local dispersion in IPyC with related SiC degradation that did not penetrate the layer, (c-e) optical micrographs of polished section showing kernel reaction with buffer and degradation in the IPyC and SiC where kernel material had protruded to the IPyC. 
16. Particle 442-A050: (a and b) oblique orthogonal x-ray tomographs showing SiC degradation through layer at tip of IPyC crack associated with edge of buffer delamination, (c) 3D semitransparent visualization showing kernel protrusion and extended $\mathrm{SiC}$ degradation along a crack in the $\mathrm{SiC}$, (d and e) optical micrographs of polished section showing $\mathrm{SiC}$ degradation at tip of IPyC crack.

17. BEC micrograph from Particle 442-A009 showing the localized SiC degradation at the tip of an IPyC crack also shown in Figure 11d.

18. BEC micrographs of Particle 442-A009 showing two magnifications of the region near the through-layer crack with a large, concentrated dispersion of high- $Z$ features in the IPyC and $\mathrm{SiC}$.

19. BEC micrographs of Particle 442-A009 for comparison to Figure 18 showing two magnifications of a region away from the through-layer crack with more typical dispersion of high-Z features.

20. BEC micrographs of Particle 442-A064 showing the distribution of uranium features in the $\mathrm{SiC}$ layer in (a) a region with an IPyC crack near the IPyC/SiC interface, and (b) an area away from any cracks in the TRISO layers.

21. SiC fracture area in Particle 442-A064 showing SEI micrograph and corresponding EDS maps of x-ray intensity indicating concentration profiles of uranium, silicon, and carbon.

22. SEI micrograph and corresponding EDS $x$-ray intensity map of the uranium concentration showing the dispersion of uranium in the buffer of Particle 442-A053.

23. Particle 442-A053 SEM micrographs showing (a) the SiC layer next to a uranium dispersion lobe with significant $\mathrm{SiC}$ attack and (b) the $\mathrm{SiC}$ layer with no adjacent uranium dispersion lobe in the IPyC layer.

24. SEM analysis of Particle 442-A053 showing (a) close-up SEI micrograph of degraded region surrounding a $\mathrm{SiC}$ crack filled with a dense material, and (b) close-up BEC micrograph confirming high- $\mathrm{Z}$ features flanking the degraded region and low- $\mathrm{Z}$ material nearest the crack.

25. SEM/EDS analysis of Particle 442-A053 showing an overview SEI micrograph of the region surrounding a through-layer crack in the $\mathrm{SiC}$ and corresponding EDS x-ray intensity maps indicating relative concentrations of uranium, silicon, and carbon.

26. SEI micrograph of Particle 442-A072 showing an area where the kernel protrusion contacted the IPyC layer; the marked zones ( 1 and 2 ) indicate regions investigated by the EDS x-ray mapping presented in Figure 27 and Figure 28.

27. Particle 442-A072 EDS analysis showing SEI micrograph of Figure 26 Zone 1 and corresponding EDS $x$-ray intensity maps indicating relative concentrations of the most prominent elements.....

28. Particle 442-A072 EDS analysis showing SEI micrograph of Figure 26 Zone 2 and corresponding EDS $x$-ray intensity maps indicating relative concentrations of the most prominent elements.....

29. Particle 442-A072 BEC micrographs showing (a) the SiC layer next to a uranium dispersion lobe with significant $\mathrm{SiC}$ attack and (b) the $\mathrm{SiC}$ layer with no adjacent uranium dispersion lobe in the IPyC layer.

30. SEM analysis of a SiC decomposition area in Particle 442-A050 showing SEI micrograph and corresponding EDS x-ray intensity maps indicating relative concentrations of uranium, silicon, and carbon. The linear features observed in the SEI micrograph are due to delamination of the $\sim 8$-nm-thick carbon coating applied to reduce charging of the sample during analysis.

31. Particle 442-A050 BEC micrographs showing SiC layer not adjacent to a crack in the IPyC layer.

32. Optical micrographs of particles that retained cesium: (a) Particle 442-A074 with high silver retention, and (b) Particle 442-A016 with low silver retention. 
33. Particle 442-A063 SEM analysis showing (a and c) SEI versus (b and d) BEC micrographs at two magnifications; some typically-observed features are identified.

34. Optical micrograph of Particle 442-A063 showing $\mathrm{SiC}$ degradation at $\mathrm{OPyC} / \mathrm{SiC}$ interface.

35. Comparison of BEC micrographs of TRISO layers from particles in Table 7 highlighting the interaction of fission products with the $\mathrm{SiC}$ as a function of silver retention

36. SEI micrograph of Particle 442-A055 showing regions of $\mathrm{SiC}$ degradation.

37. SEM/EDS analysis of IPyC and SiC layers in Particle 442-A074 showing SEI micrograph and corresponding EDS x-ray intensity maps of relative silicon, ruthenium, rhodium, palladium, and uranium concentrations.

38. SEM/EDS analysis of IPyC and SiC layers in Particle 442-A046 showing SEI micrograph and corresponding EDS x-ray intensity maps of relative silicon, ruthenium, rhodium, palladium, and uranium concentrations.

39. SEM/EDS analysis of IPyC and SiC layers in Particle 442-A063 showing SEI micrograph and corresponding EDS x-ray intensity maps of relative silicon, ruthenium, rhodium, palladium, and uranium concentrations.

40. SEM/EDS analysis of IPyC and SiC layers in Particle 442-A016 showing SEI micrograph and corresponding EDS x-ray intensity maps of relative silicon, ruthenium, rhodium, palladium, and uranium concentrations.

41. SEM/EDS analysis of IPyC and SiC layers in Particle 442-A055 showing SEI micrograph and corresponding EDS x-ray intensity maps of relative silicon, ruthenium, rhodium, palladium, and uranium concentrations.

42. SEM/EDS analysis of IPyC and SiC layers in Particle 442-A059 showing SEI micrograph and corresponding EDS x-ray intensity maps of relative silicon, ruthenium, rhodium, palladium, and uranium concentrations. 


\section{LIST OF TABLES}

1. Distribution of radioactive isotopes detected in the CCCTF furnace internals after the Compact 4-4-2 loose-particle furnace test.

2. Analysis of measurement bias after recalibration for activity of several gamma-emitting isotopes in as-irradiated Compact 4-4-2 particles

3. Activity in $\mathrm{Bq} /$ particle of gamma-emitting isotopes in particles before safety test...............................12

4. Activity in $\mathrm{Bq}$ /particle of gamma-emitting isotopes in particles after safety test................................15

5. Fraction of gamma-emitting isotopes retained in particles after safety test ........................................17

6. Activity in Bq/particle of gamma-emitting isotopes in graphite trays (Figure 1).................................19

7. Description of the six particles investigated by optical microscopy and SEM analysis that retained cesium (as well as ruthenium, antimony, and cerium), but exhibited varied retention of silver or europium. 


\section{ACRONYMS}

3D Three-dimensional

AGR Advanced Gas Reactor Fuel Development and Qualification Program

AGR-1 First AGR Program irradiation experiment

ART

ATR

Advanced Reactor Technologies

BEC

Advanced Test Reactor (at INL)

BWXT

Backscattered-electron composition

CCCTF

BWX Technologies

DOE-NE

Core Conduction Cooldown Test Facility (at ORNL)

EDS

Department of Energy Office of Nuclear Energy

FACS

Energy-dispersive spectroscopy

FB-CVD Fluidized-bed chemical vapor deposition (coating furnace)

FIMA

GMT

Fissions per initial metal atom

HFEF

Greenwich Mean Time

HTGR

Hot Fuel Examination Facility (at INL)

IFEL

High Temperature Gas-cooled Reactor

IMGA

INL

IPyC

Irradiated Fuels Examination Laboratory (ORNL Building 3525)

$\mathrm{M} / \mathrm{C}$

Idaho National Laboratory

Inner pyrolytic carbon (layer)

OPyC

Measured versus calculated ratio

ORNL Oak Ridge National Laboratory

PIE Post-irradiation examination

SD Standard deviation

SEI Secondary-electron imaging

SEM Scanning electron microscope

$\mathrm{SiC} \quad$ Silicon carbide (layer)

TAVA Time-averaged and volume-averaged (compact irradiation temperature)

TRISO Tristructural isotropic (coated particles)

Z

Atomic number 


\section{ACKNOWLEDGMENTS}

This work was sponsored by the U.S. Department of Energy, Office of Nuclear Energy, through the Idaho National Laboratory Advanced Reactor Technologies Technology Development Office as part of the Advanced Gas Reactor Fuel Development and Qualification Program. Analysis of leach solutions and Core Conduction Cooldown Test Facility furnace components was provided by the Oak Ridge National Laboratory Nuclear Analytical Chemistry \& Isotopics Laboratory. Hot cell activities were supported by the staff of the Oak Ridge National Laboratory Irradiated Fuels Examination Laboratory (IFEL). 
ORNL/TM-2015/161-R0 


\section{INTRODUCTION}

Post-irradiation examination (PIE) is being performed in support of tristructural isotropic (TRISO) coated particle fuel development and qualification for High Temperature Gas-cooled Reactors (HTGRs). This work is sponsored by the Department of Energy Office of Nuclear Energy (DOE-NE) through the Advanced Reactor Technologies (ART) Office under the Advanced Gas Reactor Fuel Development and Qualification (AGR) Program [Petti et al. 2010]. The AGR-1 experiment was the first in a series of TRISO fuel irradiation tests initiated in 2006. The AGR-1 TRISO particles and fuel compacts were fabricated at Oak Ridge National Laboratory (ORNL) in 2006 using laboratory-scale equipment and irradiated for 3 years in the Advanced Test Reactor (ATR) at Idaho National Laboratory (INL) to demonstrate and evaluate fuel performance under HTGR irradiation conditions. Post-irradiation examination was performed at INL and ORNL to study how the fuel behaved during irradiation, and to test fuel performance during exposure to elevated temperatures at or above temperatures that could occur during a depressurized conduction cooldown event. This report summarizes safety testing and post-safety testing PIE conducted at ORNL on loose particles extracted from irradiated AGR-1 Compact 4-4-2.

Compact 4-4-2 contained AGR-1 Variant 3 TRISO particles coated in the ORNL 50-mm-diameter fluidized-bed chemical vapor deposition (FB-CVD) furnace. AGR-1 Baseline and Variant 3 fuel particles had similar characteristics, except for the silicon carbide layer ( $\mathrm{SiC}$ ), which was deposited in Variant 3 at a lower temperature using an argon diluent added to the methyltrichlorosilane and hydrogen coating gases to produce a more-equiaxed, smaller-grained polycrystalline structure. Detailed fabrication and preirradiation characterization data for the Variant 3 particles and compacts can be found in the data packages for Variant 3 particles [Hunn and Lowden 2006] and compacts [Hunn, Montgomery, and Pappano 2006], and in the AGR-1 pre-irradiation summary report [Hunn, Savage, and Silva 2012]. The AGR-1 Variant $3 \mathrm{SiC}$ microstructure is the most representative of the $\mathrm{SiC}$ being deposited for the AGR program at BWX Technologies (BWXT) with an engineering-scale 150-mm-diameter FB-CVD furnace, where argon dilution is also used to produce the SiC.

AGR-1 Compact 4-4-2 was irradiated at ATR to an average calculated burnup of $16.7 \%$ fissions per initial metal atom (FIMA) [Sterbentz 2013, 48] and an average calculated fast fluence of $3.59 \cdot 10^{25} \mathrm{n} / \mathrm{m}^{2}$ [Sterbentz 2013, 51], while at a calculated time-averaged and volume-averaged (TAVA) temperature of approximately $1024^{\circ} \mathrm{C}$ [Hawkes 2014,61 ]. After capsule disassembly in the INL Hot Fuel Examination Facility (HFEF), metrology [Demkowicz et al. 2011] and gamma scanning [Harp 2013] were performed on the compact. The compact was then shipped to ORNL for safety testing and additional PIE. Compact 4-4-2 was initially subjected to as-irradiated PIE to further study its irradiation performance [Hunn et al. 2013a]. This PIE included deconsolidation of the compact and recovery of the coated particles for gamma survey with the ORNL Irradiated Microsphere Gamma Analyzer (IMGA). Ninety randomly-selected particles from Compact 4-4-2 were gamma counted for $4 \mathrm{~h}$ each to measure their post-irradiation inventory of several convenient gamma-emitting radioisotopes. Seventy-five of these intact TRISO particles were still available in an archive and used for a loose-particle safety test in the ORNL Core Conduction Cooldown Test Facility (CCCTF).

The Compact 4-4-2 loose-particle safety test began on February 19, 2014 and ended on March 20, 2014. Standard AGR-1 safety testing involved heating compacts in flowing helium to maximum temperatures of 1600,1700 , or $1800^{\circ} \mathrm{C}$ for typically $300 \mathrm{~h}$ to study fission product ${ }^{*}$ retention at these elevated temperatures [Morris et al. 2014]; $1600^{\circ} \mathrm{C}$ is the expected maximum temperature during an HTGR depressurization conduction cooldown event, while 1700 and $1800^{\circ} \mathrm{C}$ explore the performance margin. The Compact 4-4-2 loose-particle safety test was conducted at $1800^{\circ} \mathrm{C}$ for $\sim 650 \mathrm{~h}$, in accordance with examination plan PLN-4650 [Demkowicz 2014]. One objective of this test was to investigate time-

\footnotetext{
* In this report, the term "fission product" is used in a general sense to refer to all the post-fission isotopes remaining at the end of the irradiation test. These include: isotopes directly generated by the fission process, isotopes generated by neutron activation, isotopes generated by radioactive decay, and residual uranium.
} 
dependent release of fission products from coated particles without the often dominating contributions from compact matrix release; fission products that pass through intact $\mathrm{SiC}$ during irradiation are often retained in the outer pyrolytic carbon $(\mathrm{OPyC})$ layer or matrix graphite until the compacts are heated to higher temperature during safety testing. This "matrix release" makes it difficult to determine if additional fission product release through intact $\mathrm{SiC}$ is occurring during safety testing. Another objective was to attempt to quantify the fission product release from each individual particle and examine particle microstructure for correlation to any measurable retention differences.

After completion of the safety test, the particles were examined with a standard set of PIE analyses that included measurement with the IMGA of the post-safety test gamma-emitting isotopic inventory within each particle and microstructural examination by high-resolution x-ray tomography and materialography. The equipment and methods used for the safety testing and post-safety testing PIE are summarized in the next section and have previously been described in detail [Baldwin et al. 2012; Hunn et al. 2013a].

\section{EXAMINATION PROCEDURE*}

As mentioned above, the Compact 4-4-2 loose-particle safety test was a specialized test conducted on 75 individual TRISO particles deconsolidated from the compact. The loose particles were loaded into ringshaped graphite trays (Figure 1). Each tray had fifteen single-particle sample wells that were labeled and indexed to maintain individual particle identity. Five trays were held in a stack using a threaded graphite rod and circular graphite nut, resulting in a final assembled dimension similar to a typical AGR-1 compact, such that the assembled trays could be placed in a standard CCCTF graphite fuel holder.
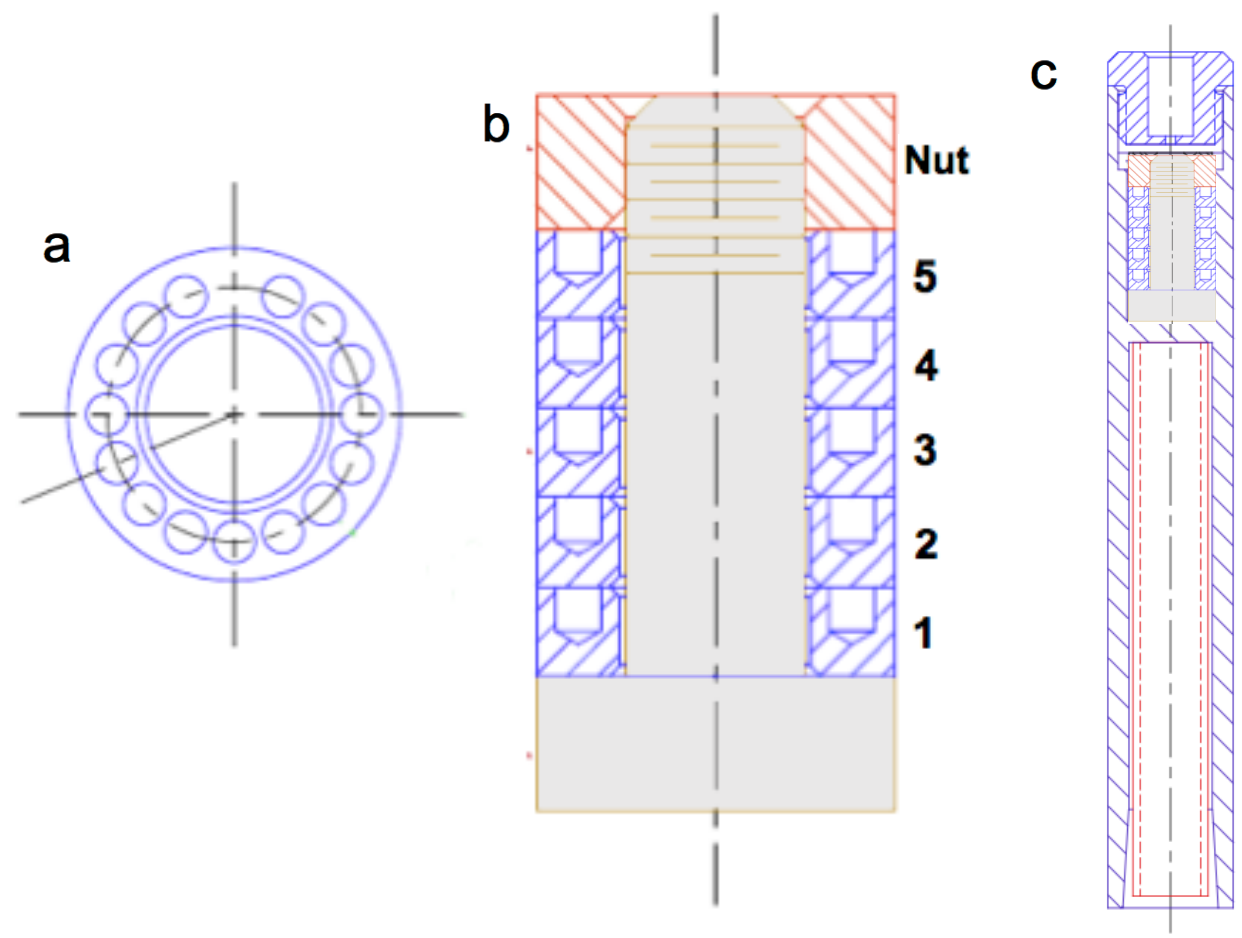

Figure 1. CCCTF fuel holder assembly for the loose-particle test: a) fifteen-particle tray, b) five-tray assembly, and c) tray assembly in standard CCCTF graphite holder.

\footnotetext{
* Some content in this section also appeared in letter reports summarizing the Compact 4-4-2 loose particle safety test [Hunn et al. 2014a] and earlier AGR-1 PIE that utilized similar post-safety test procedures [Hunn et al. 2013b].
} 
Details of temperatures and hold times used in this test are presented with the safety test results in the next section. During the test period, metallic elements released from the compacts were collected by a water-cooled cold finger at the top of the CCCTF furnace, and gaseous fission products were extracted from the helium sweep gas as it passed through a liquid nitrogen-cooled cold trap located downstream. Deposition cups attached to the in-furnace cold finger were periodically removed and analyzed by gamma spectrometry and the sweep gas trap was monitored for gamma activity throughout the run. As originally designed, the deposition cups have a high efficiency for collection of silver and cesium at $1600-1800^{\circ} \mathrm{C}$, and the sweep gas trap system has a good sensitivity to release of ${ }^{85} \mathrm{Kr}$. Time-dependent monitoring of europium and strontium (not originally part of the CCCTF design) includes a larger uncertainty, due to the fact that these elements can be retained by the graphite and tantalum furnace internals; analysis of the CCCTF furnace internals at the end of each test is important for accurate assessment of the total cumulative release of these elements. For a typical AGR- $11600^{\circ} \mathrm{C}$ safety test in the CCCTF, the fraction of europium that eventually accumulated on the deposition cups was only $10-13 \%$, while silver collection efficiency was typically above $95 \%$.

After completion of the safety test, additional analyses were performed on the deposition cups and other CCCTF furnace internals to measure the activity of the radioactive fission products that were transferred from the particles to these various furnace components. Deposition cups were subjected to gamma spectrometry and then acid leached for measurement of stable isotopes and ${ }^{90} \mathrm{Sr}$. The graphite components (Figure 1) were burned and the ash leached with hot nitric acid. The tantalum components were also acid leached to remove fission products from the exposed surfaces. Leach solutions were analyzed by gamma spectrometry for gamma-emitting radioisotopes, mass spectrometry for actinides and stable isotopes, and beta spectrometry for ${ }^{90} \mathrm{Sr}$ after chemical separation to isolate the strontium. The average deposition cup collection efficiency was determined for each fission product by measuring the total release from the particles (summing the inventories measured on the graphite tray assembly, graphite holder, tantalum furnace internals, and deposition cups) and calculating the fraction of the total that went to the cups. The average collection efficiency was then used to adjust the time-dependent deposition cup data to estimate the time-dependent fission product release from the particles.

Post-safety test PIE consisted of re-measuring the inventory of the gamma-emitting radioisotopes typically quantified with the IMGA $\left({ }^{106} \mathrm{Ru},{ }^{110 \mathrm{~m}} \mathrm{Ag},{ }^{125} \mathrm{Sb},{ }^{134} \mathrm{Cs},{ }^{137} \mathrm{Cs},{ }^{144} \mathrm{Ce}\right.$, and $\left.{ }^{154} \mathrm{Eu}\right)$, and then performing microstructural analysis of selected particles. Microstructural analysis included x-ray imaging and materialographic inspection.

Three-dimensional (3D) microstructural analysis was achieved using a high-resolution x-ray tomography system specifically designed for imaging TRISO-coated particles. This system can image coating structure with resolution close to $1 \mu \mathrm{m}$, and has been used during AGR-1 fuel development to characterize and understand defect structures in unirradiated, as-fabricated particles in a manner not previously available. For AGR-1 PIE, a shielded container was designed for mounting irradiated particles on the x-ray tomography stage. This shielded container allows single particles to be removed from the hot cell and transported to the tomography instrument for imaging. The shielding also reduces gamma radiation interference in the detector and electronics. Radiographic image sets using 3200 particle orientations were acquired to support high-resolution 3D tomographic reconstruction of the irradiated particle internal microstructure.

Mechanical polishing to reveal particle cross sections was achieved by mounting particles in epoxy and grinding/polishing with a Buehler Minimet 1000 [Hunn et al. 2013a, 30-32]. Vacuum back-potting was used to prevent damage to the internal structure and improve the quality of the final polish. Kernels and coating layers were imaged using an optical microscope. Scanning electron microscopy (SEM) was performed on some samples to provide additional microstructural information and energy-dispersive spectroscopy (EDS) was used to identify clustered fission products. 


\section{FURNACE TESTING RESULTS*}

The overall temperature profile for the safety test and the time-dependent, cumulative releases of krypton and cesium are shown in Figure 2. The inventory releases are plotted in comparison to the average calculated particle inventory [Sterbentz 2013]. Significant krypton release is indicative of the failure of all three outer TRISO coatings to remain gas-tight. The ${ }^{85} \mathrm{Kr}$ release shown in Figure 2 indicates that there were three particles with TRISO failure during the initial heat-up from $1250^{\circ} \mathrm{C}$ to $1800^{\circ} \mathrm{C}$ and two more particles failed while at $1800^{\circ} \mathrm{C}$ (the first after about $125 \mathrm{~h}$ at $1800^{\circ} \mathrm{C}$ and the second after about $540 \mathrm{~h}$ at $1800^{\circ} \mathrm{C}$ ). Significant cesium release can occur through failure of the $\mathrm{SiC}$ layer independent of pyrocarbon failure (intact pyrocarbon will retain krypton, but not cesium). Within the time resolution available from the data collection intervals (4-hour averaging for sweep gas monitoring and 12-24-hour collection periods for deposition cups), significant cesium release occurred concurrent with the initial three TRISO failures and with the fourth TRISO failure. Figure 3 shows that the average rate of cesium collection on the deposition cups was highest on the deposition cups that were in the furnace at the time of the first two krypton releases, and had dropped back to a negligible level by the end of the next cup-exchange period. This concurrent release behavior would be expected if the $\mathrm{SiC}$ was the last layer to fail or if the $\mathrm{SiC}$ and OPyC failed at the same time, as might occur in the event of a through-TRISO coating fracture. The final increase in cesium release, beginning around $450 \mathrm{~h}$ into the test, built up more gradually and appeared to precede the final TRISO failure. This release behavior probably indicates that the $\mathrm{SiC}$ failed prior to failure of the last intact pyrocarbon layer. Such a scenario might occur if the $\mathrm{SiC}$ failed by the typical mechanism observed in other safety tests, where buffer shrinkage led to cracking of the inner pyrolytic carbon (IPyC) layer and localized palladium degradation of the exposed $\mathrm{SiC}$, while the OPyC layer remained intact [Hunn et al. 2014b].

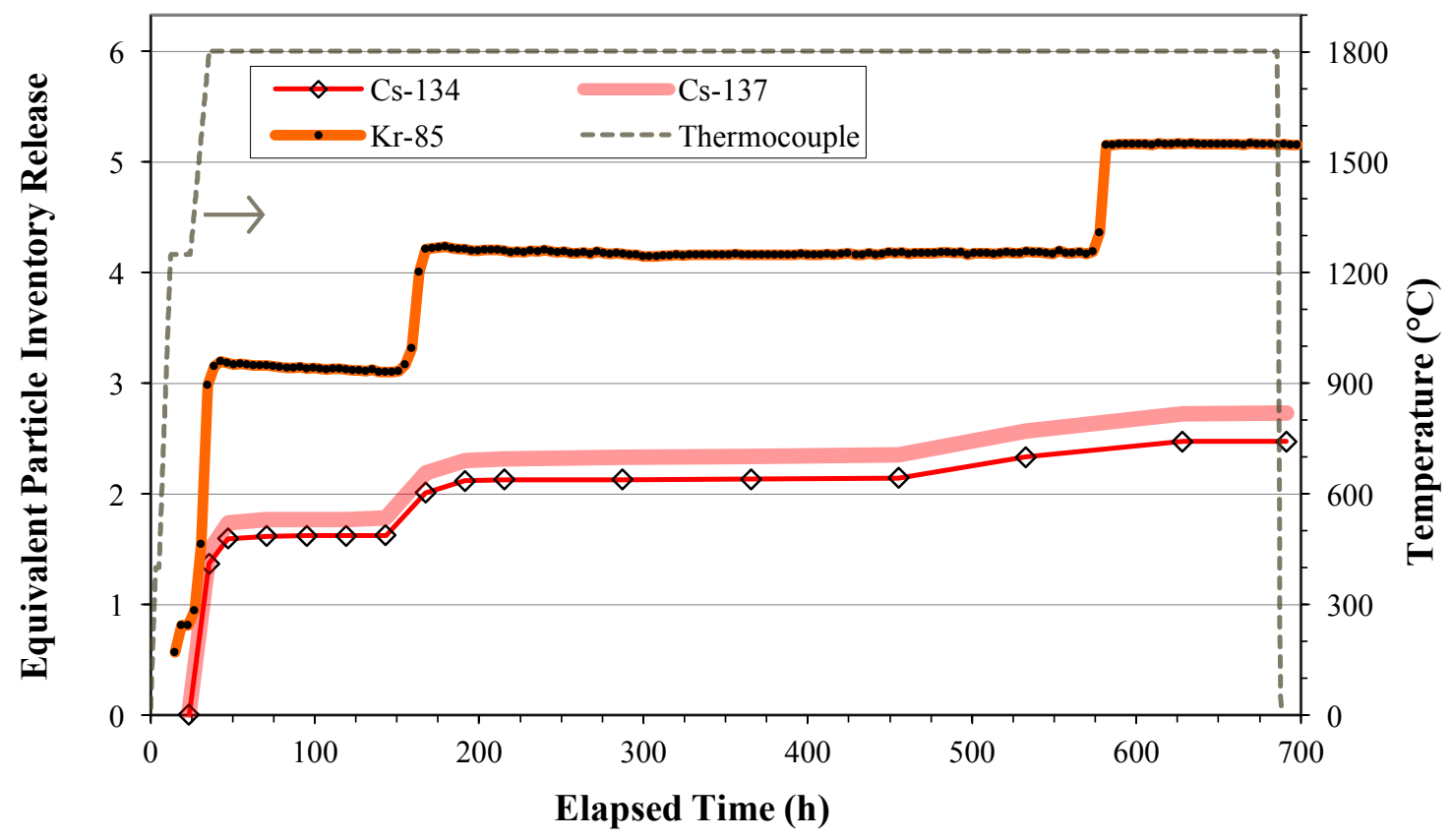

Figure 2. Cumulative release of cesium and krypton from Compact 4-4-2 loose particles at $1800^{\circ} \mathrm{C}$. Note that the magnitude of the cesium release may be off due to uncertainty in the deposition cup collection efficiency, as discussed at the end of this section.

\footnotetext{
${ }^{*}$ Some content in this section also appeared in a letter report summarizing the Compact 4-4-2 loose particle safety test [Hunn et al. 2014a].
} 


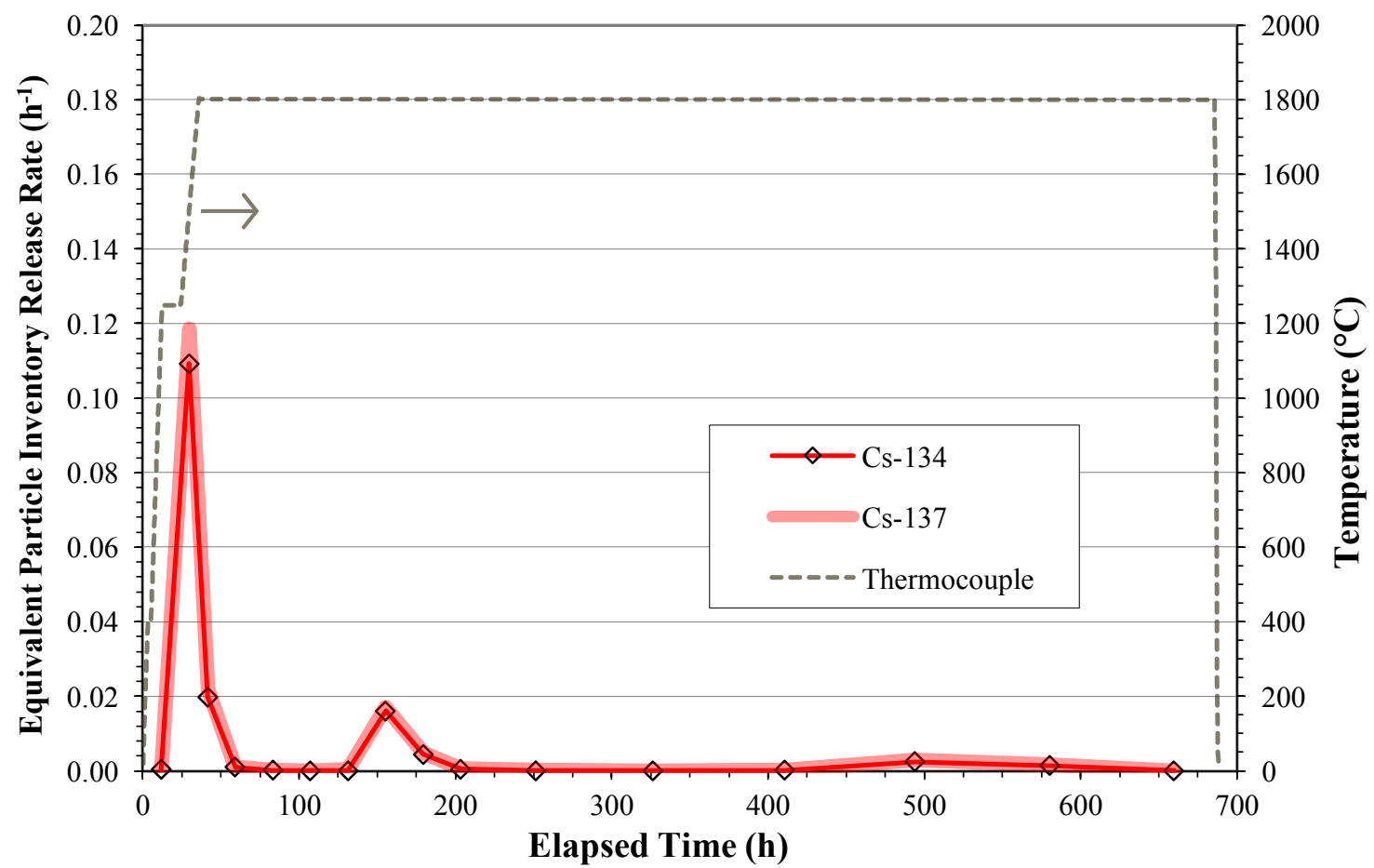

Figure 3. Average rate of cesium release during each deposition cup collection interval.

Krypton release from failed TRISO is an unusual result for AGR-1 safety testing. None of the other thirteen CCCTF tests exhibited this type of krypton release (only diffusive releases of $<25 \%$ of one particle's ${ }^{85} \mathrm{Kr}$ inventory from particles with $\mathrm{SiC}$ failure has been observed in the CCCTF). However, one $1800^{\circ} \mathrm{C}$ test in the INL Fuel Accident Condition Simulator (FACS) furnace released krypton from failed TRISO with similar release kinetics to that shown in Figure 2 [Demkowicz et al. 2015a, 323-325].

Release of some of the other measured radioisotopes is shown in Figure 4. Europium and strontium were gradually released throughout the test. Similar behavior has been observed in CCCTF tests of AGR-1 compacts and is believed to have been dominated by slow migration of these elements from the OPyC and surrounding graphite, as well as possible retention by the tantalum components. Table 1 lists the fraction of the total furnace test releases detected on various internal CCCTF furnace components at the end of the loose particle safety test and most of the europium appeared to be captured by the tantalum. The fraction of europium released during the loose particle safety test was, however, much greater than has been released in 300-hour safety tests of AGR-1 compacts, and is indicative of release through intact $\mathrm{SiC}$ during the safety test. Europium release during the shorter whole-compact safety tests has not been high enough to rule out the possibility that the observed release may have been limited to release from the matrix and $\mathrm{OPyC}$ of europium previously released through intact $\mathrm{SiC}$ during irradiation.

Table 1. Distribution of radioactive isotopes detected in the CCCTF furnace internals after the Compact 4-4-2 loose-particle furnace test

\begin{tabular}{|l||c|c|c|c|c|}
\hline Component & ${ }^{\mathbf{9 0}} \mathbf{S r}$ & ${ }^{\mathbf{1 3 4}} \mathbf{C s}$ & ${ }^{\mathbf{1 1 0 m}_{\mathbf{A g}}}$ & ${ }^{\mathbf{1 5 4}} \mathbf{E u}$ & ${ }^{\mathbf{1 5 5}} \mathbf{E u}$ \\
\hline \hline Deposition cups & $44.7 \%$ & $51.1 \%$ & $60.5 \%$ & $10.0 \%$ & $8.8 \%$ \\
\hline Tantalum parts & $52.5 \%$ & $48.9 \%$ & $39.5 \%$ & $85.8 \%$ & $87.0 \%$ \\
\hline Graphite holder & $2.8 \%$ & $<0.1 \%$ & $<0.1 \%$ & $4.0 \%$ & $4.2 \%$ \\
\hline Graphite trays & $<0.1 \%$ & $<0.1 \%$ & $<0.1 \%$ & $0.3 \%$ & $<0.1 \%$ \\
\hline
\end{tabular}




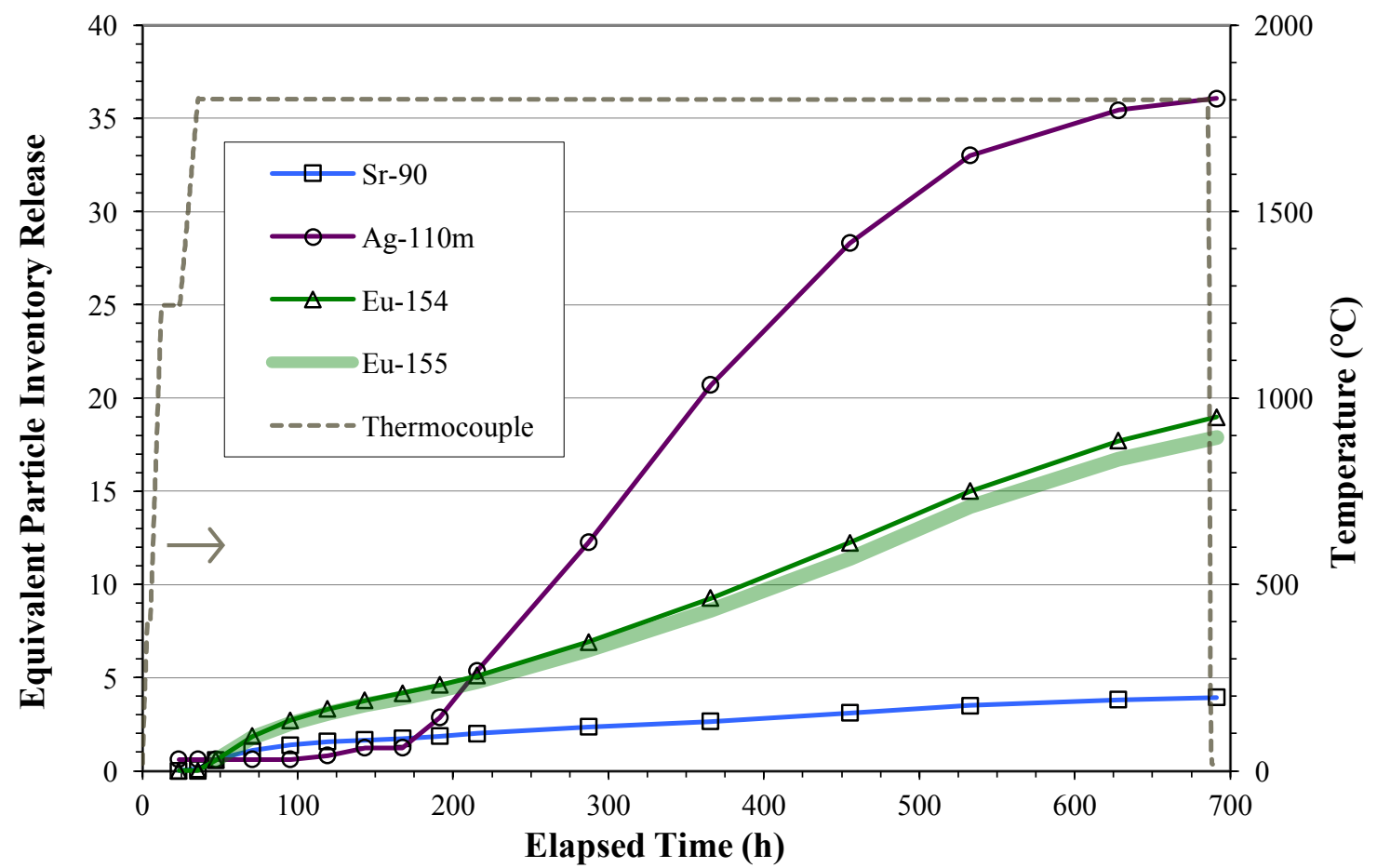

Figure 4. Cumulative release of silver, europium, and strontium from Compact 4-4-2 loose particles at $1800^{\circ} \mathrm{C}$. Note that the magnitude of the plotted release may be off due to uncertainty in the deposition cup collection efficiency, as discussed at the end of this section.

Silver release and distribution within the furnace was anomalous to typical CCCTF behavior. Silver is usually released from the compact matrix and OPyC during the initial heatup of a compact, with relatively little additional release from the compact through the remainder of the safety test. Two exceptions to this behavior have been when test temperature was not held constant and below $1600^{\circ} \mathrm{C}$ [Hunn et al. $2014 \mathrm{c}$; Hunn et al. 2015], which is not relevant here, and during the test of Compact 4-4-1, which released the typical initial burst of silver during heatup followed by a secondary increase in silver release after about $70-100 \mathrm{~h}$ at $1800^{\circ} \mathrm{C}$ (Figure 5) [Hunn et al. 2013b, 8]. It is speculated that this secondary silver release from Compact 4-4-1 may have been related to diffusive release through the finer-grained $\mathrm{SiC}$ microstructure unique to this AGR-1 Variant 3 fuel type. Compact 4-3-2 (also Variant 3) was safety tested in the INL FACS furnace and showed a similar behavior [Demkowicz et al. 2015a, 327]. The Compact 4-4-2 loose particles did not release much silver during initial heatup (Figure 4); this indicates that there was not significant silver inventory remaining in the OPyC of the deconsolidated and acid leached particles. The onset of significant silver release from the Compact 4-4-2 particles around $170 \mathrm{~h}$ into the test is reminiscent of the Variant 3 compacts safety tested at $1800^{\circ} \mathrm{C}$. Figure 5 shows that additional silver was released from Compact 4-4-1 at essentially a constant rate for the last $100 \mathrm{~h}$ at $1800^{\circ} \mathrm{C}$ (from roughly 230 to $330 \mathrm{~h}$ into the test). Figure 4 shows a similar constant silver release from the Compact 4-4-2 loose particles over the same time interval, followed by an eventual tapering off in the release rate; this tapering off was a result of depletion of the silver inventory in the 75-particle test sample. 


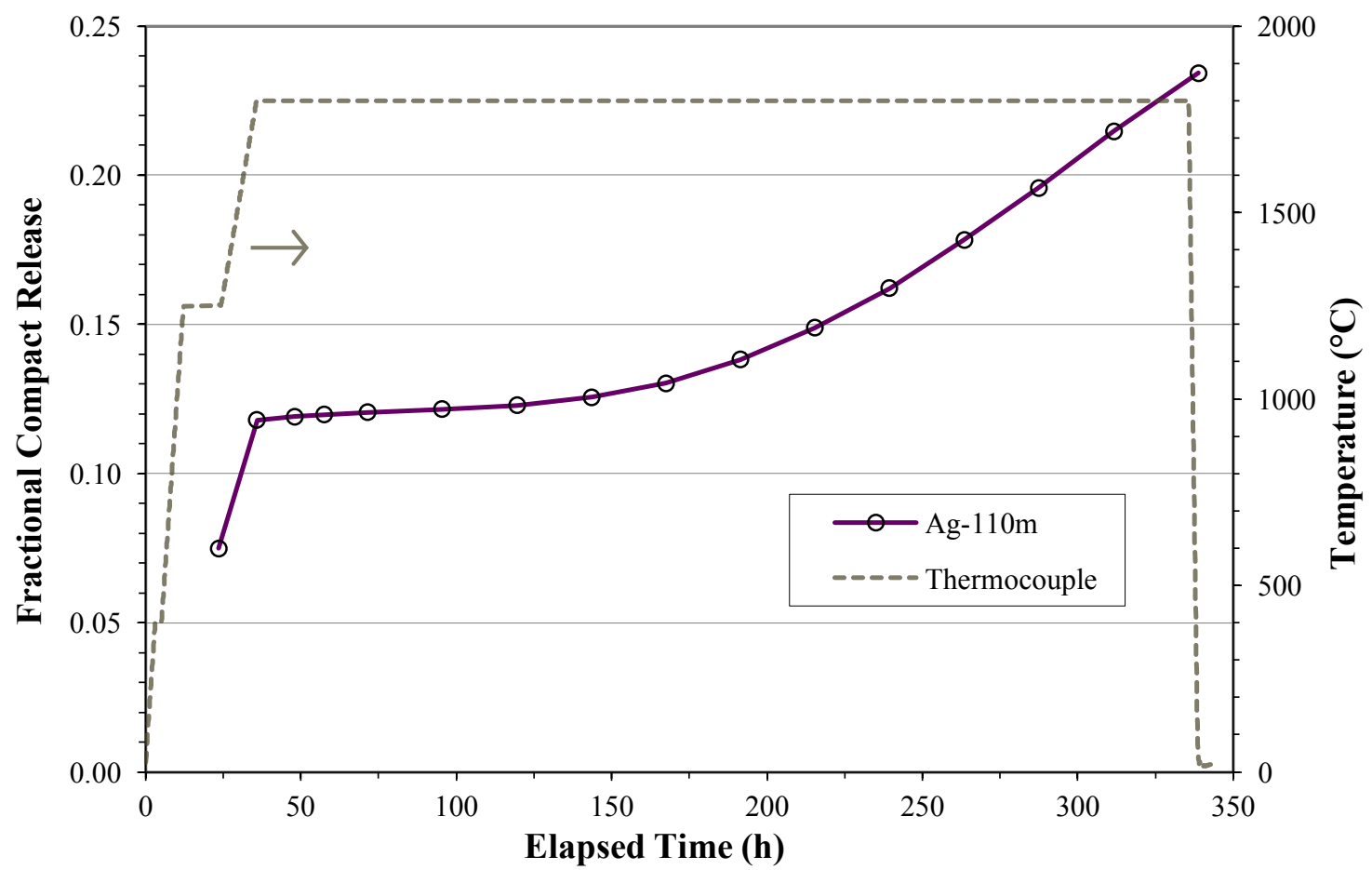

Figure 5. Time-dependent cumulative release of silver from $1800^{\circ} \mathrm{C}$ safety test of Compact 4-4-1.

An unusually large fraction $\left(39.5 \%\right.$ ) of the total detected ${ }^{110 \mathrm{~m}} \mathrm{Ag}$ (Table 1) was located at the top of the furnace on the outside surface of the tantalum gas inlet line (in a cooler region of the furnace above the deposition cup); other $1800^{\circ} \mathrm{C} \mathrm{CCCTF} \mathrm{safety} \mathrm{tests} \mathrm{have} \mathrm{exhibited}>99 \%$ average deposition cup collection efficiency for silver. The reduced silver collection efficiency for the Compact 4-4-2 looseparticle test appears to be related to a higher than normal deposition cup temperature. Post-safety test measurements of the deposition cup temperature have shown that the cups used for this test had a larger gap between the bottom of the cup and the water-cooled cold finger, and this gap impacted the cooling of the cup surface. Cesium and europium were also detected on the gas line at the top of the furnace; these elements also exhibited lower than expected collection efficiencies, compared to previous tests. The Compact 4-2-2 safety test [Hunn et al. 2014c; Hunn et al. 2015] followed the loose-particle test and showed a similar cup collection problem. It is unlikely that fission products collected at the top of the furnace are transported back down to the deposition cup, which is closer to the heated zone and at a higher temperature (Figure 6). Therefore, this anomalous reduced collection efficiency should not impact the general conclusions drawn from these two furnace tests, but may impact the quantitative analysis of the total release due to larger uncertainty in the measurement of fission products that may have collected at the top of the furnace can.

This quantitative uncertainty appears to be reflected in the total estimated amount of cesium released during the safety test. Figure 2 indicates five failed-TRISO particles with associated ${ }^{85} \mathrm{Kr}$ release essentially equivalent to the total ${ }^{85} \mathrm{Kr}$ inventory from those particles. However, the total cesium release plotted in Figure 2 is less than three particles' worth. As discussed in the next sections, IMGA survey and subsequent microstructural analyses verified the presence of these five failed-TRISO particles and the cesium inventory retained in those particles was nearly zero. Thus only about half the cesium release can be accounted for by the analyses on the furnace components. This suggests either an error in the analysis of the furnace internals or cesium deposition elsewhere in the furnace (such as the furnace lid, cold finger, or exhaust lines, which were not analyzed). The cumulative release of ${ }^{110 \mathrm{~m}} \mathrm{Ag}$ plotted in Figure 4 also appears to be erroneously-low compared to the IMGA data reported in Section 4, although the magnitude of this error is difficult to determine due to the high measurement uncertainty and detection threshold for ${ }^{110 \mathrm{~m}} \mathrm{Ag}$. 


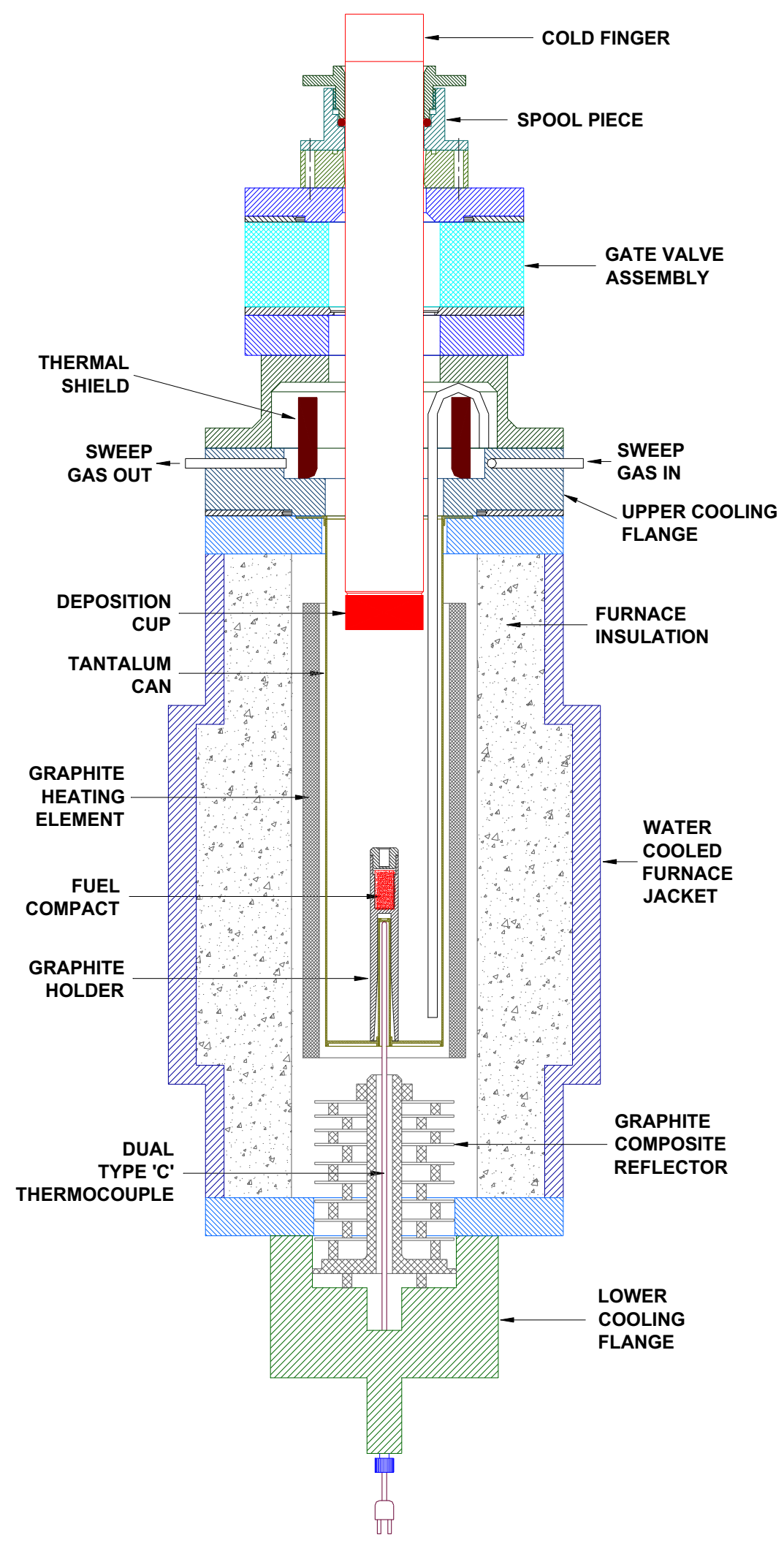

Figure 6. Schematic of the CCCTF furnace [Baldwin et al., 2012]. 


\section{IMGA RESULTS}

As part of the initial PIE on as-irradiated Compact 4-4-2 [Hunn et al. 2013a], a randomly-selected sample of 90 particles from Compact 4-4-2 was loaded into individual vials and subjected to four-hour gamma scanning to obtain a quantitative analysis of several key radioisotopes $\left({ }^{106} \mathrm{Ru},{ }^{110 \mathrm{~m}} \mathrm{Ag},{ }^{125} \mathrm{Sb},{ }^{134} \mathrm{Cs},{ }^{137} \mathrm{Cs}\right.$, ${ }^{144} \mathrm{Ce}$, and ${ }^{154} \mathrm{Eu}$ ). By handling particles in separate vials, the individual identity of each particle could be maintained throughout the remainder of the PIE. Thirteen particles from the 90-particle randomlyselected sample were used for x-ray and materialography to study the as-irradiated microstructure; 75 of the remaining 77 particles were used for the loose-particle furnace test. In the initial PIE IMGA data, there was no indication of cesium release from failed $\mathrm{SiC}$ in any of the surveyed Compact 4-4-2 particles (Figure 7 shows no outliers in the ${ }^{137} \mathrm{Cs}$ distribution) and only ${ }^{110 \mathrm{~m}} \mathrm{Ag}$ exhibited a measurably-reduced radioisotope inventory (Figure 8 shows a low-end tail in the ${ }^{110 \mathrm{~m}} \mathrm{Ag}$ distribution that indicates silver was released during irradiation through intact $\mathrm{SiC}$ in at least $15 \%$ of the 90 -particle sample). Leach-burn-leach analysis showed that at least $0.06 \%$ of the calculated compact inventory of ${ }^{154} \mathrm{Eu}$ passed through intact $\mathrm{SiC}$ during irradiation [Hunn et al. 2013a, 12], but this small release was not enough to be detected with IMGA as a reduction in the individual particle inventory. Figure 9 shows a tight ${ }^{154} \mathrm{Eu}$ distribution, indicating uniform retention behavior, but centered at a measured versus calculated ratio $(\mathrm{M} / \mathrm{C})$ less than one. However, the determination of an average ${ }^{154} \mathrm{Eu}$ inventory around 0.86 does not indicate europium release; similar mean values have been observed from IMGA analysis of particles from other AGR-1 compacts, and gamma spectrometry performed on the AGR-1 compacts before deconsolidation [Harp 2013, 11-12] consistently showed less ${ }^{154} \mathrm{Eu}$ than predicted, in spite of the relatively-low quantities found outside of the compacts in the capsule components [Demkowicz et al. 2014].

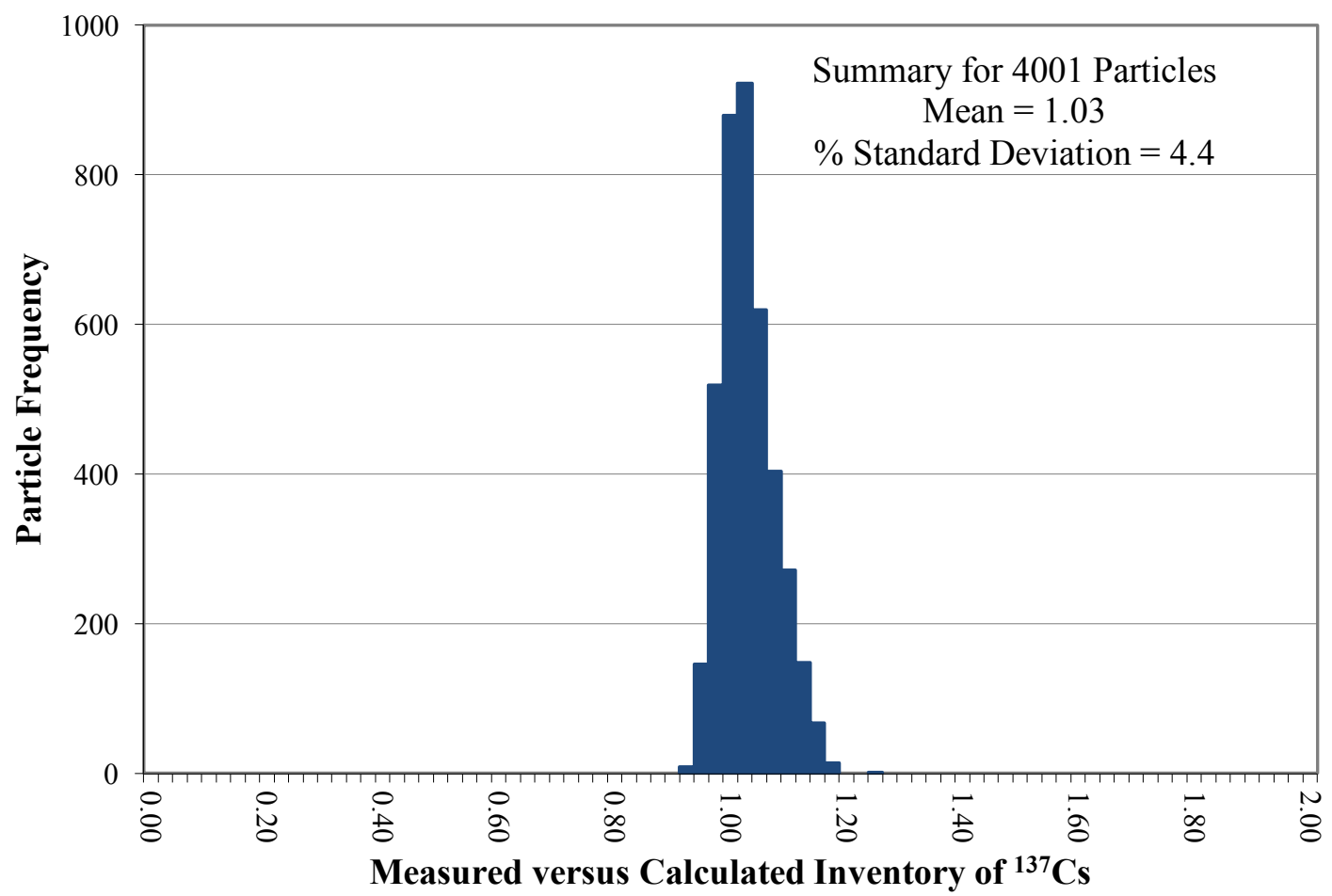

Figure 7. Ratio of ${ }^{137} \mathrm{Cs}$ in 4001 particles deconsolidated from as-irradiated Compact 4-4-2 versus the calculated inventory, adjusted for variation in fissionable material and burnup with the measured ${ }^{144} \mathrm{Ce}$ activity [Hunn et al. 2013a, 23]. 


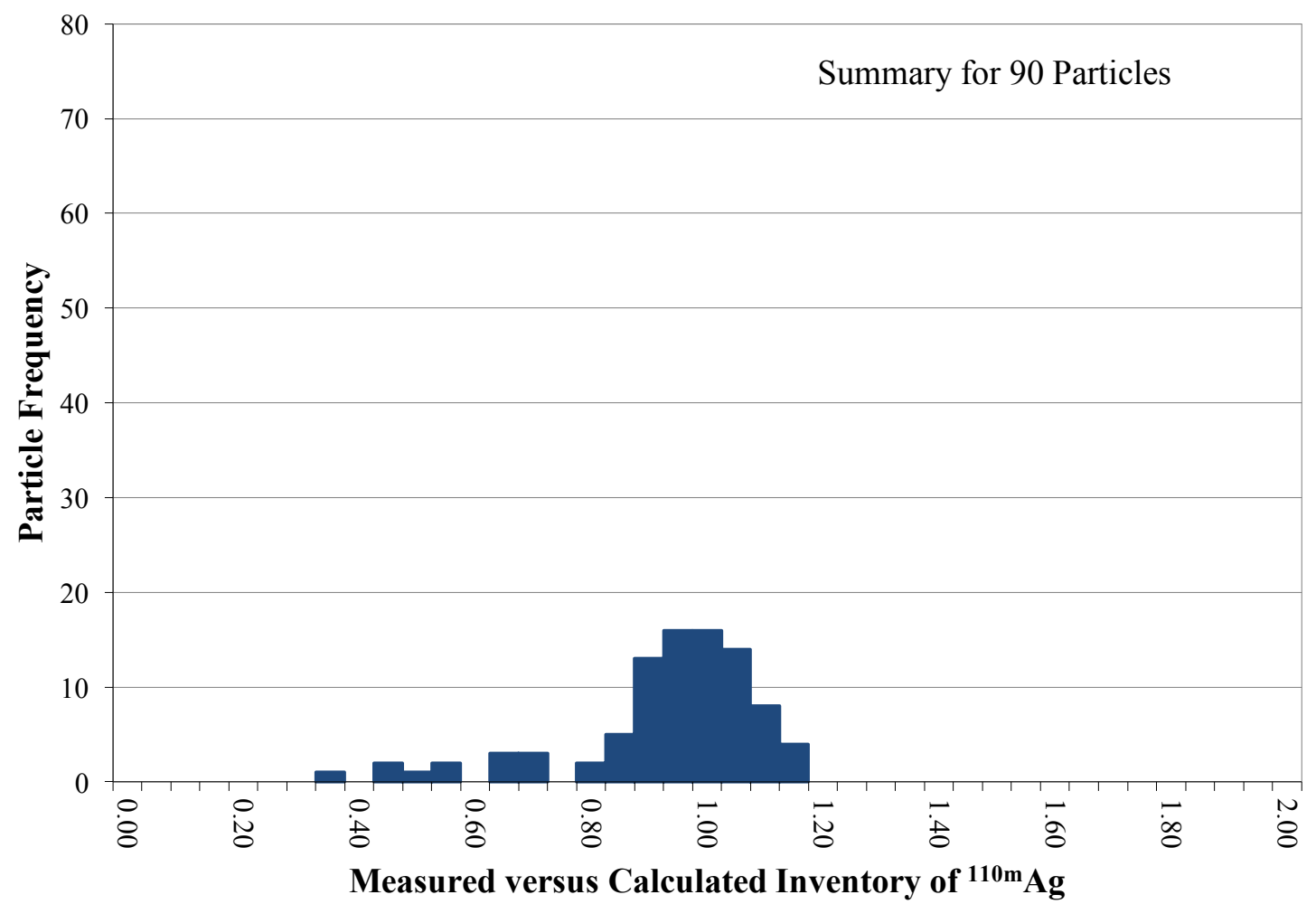

Figure 8. Ratio of ${ }^{110 \mathrm{~m}} \mathrm{Ag}$ in 90 as-irradiated Compact 4-4-2 particles versus calculated inventory, adjusted for variation in fissionable material and burnup with the measured ${ }^{137} \mathrm{Cs}$ activity [Hunn et al. 2013a, 25].

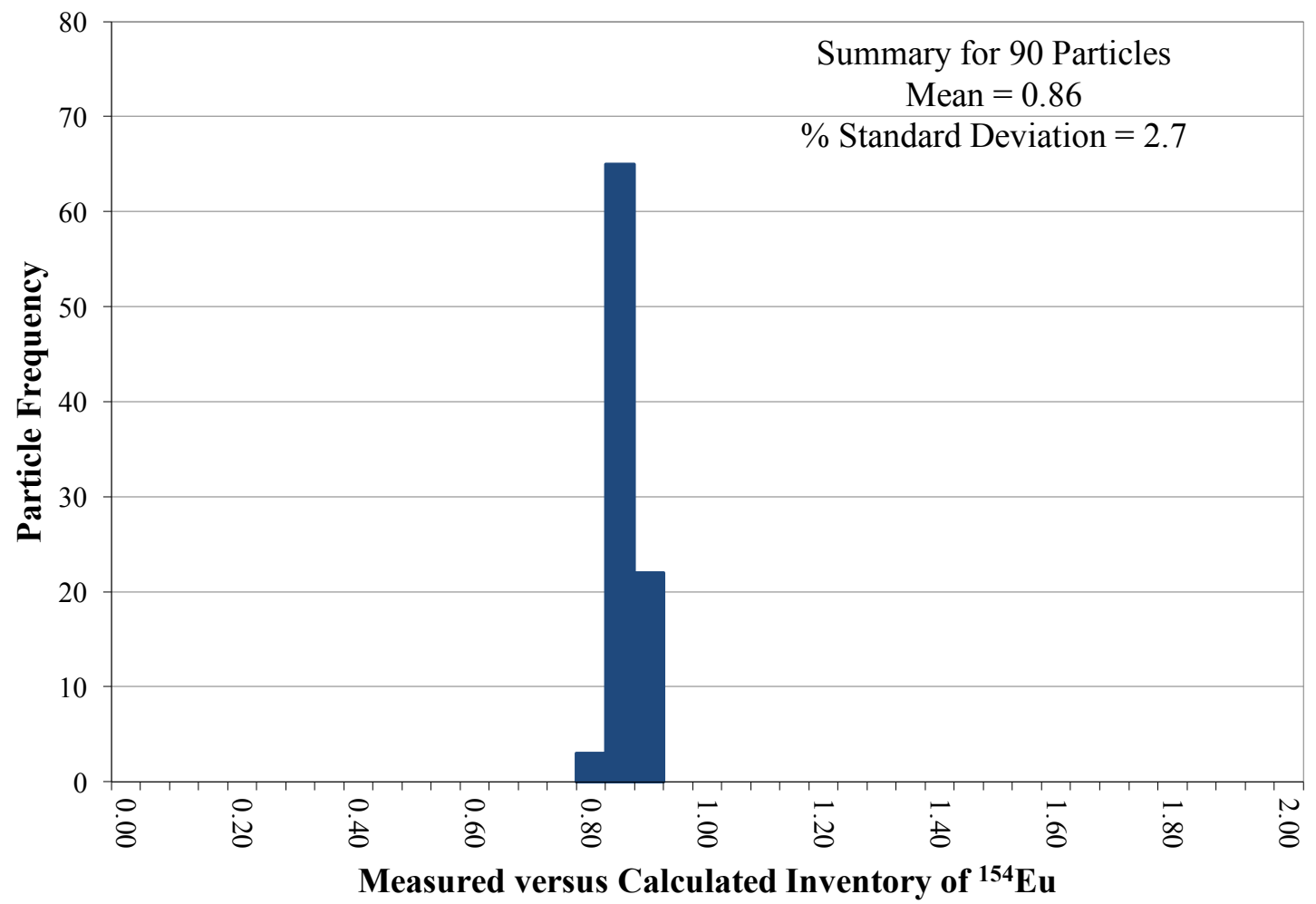

Figure 9. Ratio of ${ }^{154} \mathrm{Eu}$ in 90 as-irradiated Compact 4-4-2 particles versus calculated inventory, adjusted for variation in fissionable material and burnup with the measured ${ }^{137} \mathrm{Cs}$ activity [Hunn et al. 2013a, 24]. 
The initial gamma counting of Compact 4-4-2 as-irradiated particles was performed in 2011; gamma counting for the loose particle furnace test was performed in 2014. In 2013, the sample-detector distance was changed from $50 \mathrm{~cm}$ to $35 \mathrm{~cm}$ in response to the reduction in AGR-1 particle activity due to radioactive decay, and the IMGA system was recalibrated for the closer geometry. Detail on the IMGA calibration is included in a description of the IMGA in the AGR-1 Irradiated Compact 4-4-2 PIE Report [Hunn et al. 2013a, 19] and a table of detector efficiencies for both $50-\mathrm{cm}$ and $35-\mathrm{cm}$ detector geometries is discussed in the PIE report for Compacts 5-2-3 and 5-2-1 [Hunn et al. 2014d, 11]. Before starting the furnace test, twelve as-irradiated Compact 4-4-2 particles were re-measured with the IMGA using the $35-\mathrm{cm}$ geometry and a six-hour counting time; results were compared to the original data acquired at $50 \mathrm{~cm}$ to quantify any systematic bias that might be introduced by uncertainty in the two calibrations. Table 2 lists the two data sets after decay-correcting all the measured activities to one day after the end of irradiation (11/07/2009 at 1200 GMT). The average bias for each radioisotope (average ratio of second measurement at $35 \mathrm{~cm}$ to first measurement at $50 \mathrm{~cm}$ ) is given at the bottom of the table. This average bias was used to adjust the original $50-\mathrm{cm}$ dataset for direct comparison to the post-safety test IMGA data; the adjusted pre-safety test IMGA data is provided in Table 3. The standard deviation (SD) values reported in Table 2 indicate the bias was very consistent for every measured isotope except ${ }^{110 \mathrm{~m}} \mathrm{Ag}$. The lack of repeatability for ${ }^{110 \mathrm{~m}} \mathrm{Ag}$ was due to the very-low activity at the time of the second measurement, which resulted in a large uncertainty multiplier when the decay correction was applied (measurement was made over 6 half-lives after the end of irradiation).

Table 2. Analysis of measurement bias after recalibration for activity of several gamma-emitting isotopes in as-irradiated Compact 4-4-2 particles

\begin{tabular}{|c|c|c|c|c|c|c|c|}
\hline Particle & ${ }^{106} \mathrm{Ru}$ & ${ }^{110 m} \mathrm{Ag}$ & ${ }^{125} \mathrm{Sb}$ & ${ }^{134} \mathrm{Cs}$ & ${ }^{137} \mathrm{Cs}$ & ${ }^{144} \mathrm{Ce}$ & ${ }^{154} \mathbf{E u}$ \\
\hline 442-A003 & $\begin{array}{l}43 \mathrm{E} 7 \\
1.44 \mathrm{E} 7\end{array}$ & $\frac{4.79 \mathrm{E} 4}{5.01 \mathrm{E} 4}$ & $\frac{2.27 \mathrm{E} 5}{2.51 \mathrm{E} 5}$ & $\begin{array}{r}4.84 \mathrm{E} 6 \\
4.90 \mathrm{E} 6\end{array}$ & $\begin{array}{r}4.24 \mathrm{E} 6 \\
\quad 4.44 \mathrm{E} 6\end{array}$ & $\begin{array}{l}5.92 \mathrm{E} 7 \\
5.98 \mathrm{E} 7\end{array}$ & $\begin{array}{r}1.35 \mathrm{E} 5 \\
1.42 \mathrm{E} 5\end{array}$ \\
\hline 442-A012 & $\frac{20 \mathrm{E} 7}{1.21 \mathrm{E} 7}$ & $\frac{2.15 \mathrm{E} 4}{3.73 \mathrm{E} 4}$ & $\frac{2.02 \mathrm{E} 5}{2.21 \mathrm{E} 5}$ & $\frac{4.50 \mathrm{E} 6}{4.56 \mathrm{E} 6}$ & $\frac{3.86 \mathrm{E} 6}{4.03 \mathrm{E} 6}$ & $\frac{5.16 \mathrm{E} 7}{5.21 \mathrm{E} 7}$ & $\frac{1.27 \mathrm{E} 5}{1.32 \mathrm{E} 5}$ \\
\hline $442-$ & $\mid \begin{array}{l}1.27 \mathrm{E} 7 \\
1.28 \mathrm{E} 7\end{array}$ & $\frac{4.08 \mathrm{E} 4}{4.71 \mathrm{E} 4}$ & $\frac{2.05 \mathrm{E} 5}{2.24 \mathrm{E} 5}$ & $\frac{4.81 \mathrm{E} 6}{4.89 \mathrm{E} 6}$ & $\frac{3.95 \mathrm{E} 6}{4.16 \mathrm{E} 6}$ & $5 \frac{5.05 \mathrm{E} 7}{5.15 \mathrm{E} 7}$ & $\frac{1.35 \mathrm{E} 5}{1.42 \mathrm{E} 5}$ \\
\hline 442-A027 & $\begin{array}{r}1.30 \mathrm{E} 7 \\
1.31 \mathrm{E} 7\end{array}$ & $\frac{4.89 \mathrm{E} 4}{4.57 \mathrm{E} 4}$ & $\frac{2.14 \mathrm{E} 5}{2.24 \mathrm{E} 5}$ & $\frac{4.40 \mathrm{E} 6}{4.50 \mathrm{E} 6}$ & $\begin{array}{r}3.84 \mathrm{E} 6 \\
4.05 \mathrm{E} 6\end{array}$ & $\frac{5.15 \mathrm{E} 7}{5.25 \mathrm{E} 7}$ & $\frac{1.31 \mathrm{E} 5}{1.34 \mathrm{E} 5}$ \\
\hline 442-A033 & $\begin{array}{l}1.06 \mathrm{E} 7 \\
1.07 \mathrm{E} 7\end{array}$ & $\frac{4.12 \mathrm{E} 4}{3.42 \mathrm{E} 4}$ & $\frac{1.74 \mathrm{E} 5}{1.93 \mathrm{E} 5}$ & $\frac{4.00 \mathrm{E} 6}{4.10 \mathrm{E} 6}$ & $\frac{3.37 \mathrm{E} 6}{3 .}$ & $\frac{4.42 \mathrm{E} 7}{4.46 \mathrm{E} 7}$ & $\frac{1.13 \mathrm{E} 5}{1.20 \mathrm{E} 5}$ \\
\hline $442-$ & \begin{tabular}{|l}
$1.27 \mathrm{E} 7$ \\
$1.27 \mathrm{E} 7$
\end{tabular} & $\frac{3.91 \mathrm{E} 4}{4.79 \mathrm{E} 4}$ & $\frac{2.03 \mathrm{E} 5}{2.25 \mathrm{E} 5}$ & $\frac{4.46 \mathrm{E} 6}{4.54 \mathrm{E} 6}$ & $\begin{array}{r}3.90 \mathrm{E} 6 \\
4.09 \mathrm{E} 6\end{array}$ & $\frac{5.16 \mathrm{E} 7}{5.26 \mathrm{E} 7}$ & $\frac{1.32 \mathrm{E} 5}{1.36 \mathrm{E} 5}$ \\
\hline 442 & $\begin{array}{r}1.32 \mathrm{E} 7 \\
1.33 \mathrm{E} 7\end{array}$ & $\frac{4.85 \mathrm{E} 4}{4.83 \mathrm{E} 4}$ & $\frac{2.14 \mathrm{E} 5}{2.31 \mathrm{E} 5}$ & $\frac{4.84 \mathrm{E} 6}{4.94 \mathrm{E} 6}$ & $\frac{4.15 \mathrm{E} 6}{4.32 \mathrm{E} 6}$ & $5 \quad 5.52 \mathrm{E} 7$ & $\begin{array}{r}1.35 \mathrm{E} 5 \\
1.43 \mathrm{E} 5\end{array}$ \\
\hline 44 & $\begin{array}{r}1.35 \mathrm{E} 7 \\
1.34 \mathrm{E} 7\end{array}$ & $\frac{4.88 \mathrm{E} 4}{4 .}$ & $\frac{2.16 \mathrm{E} 5}{2}$ & $5.15 \mathrm{E} 6$ & $\frac{4.15 \mathrm{E} 6}{4.27 \mathrm{E} 6}$ & $\frac{5.15 \mathrm{E} 7}{5.16 \mathrm{E} 7}$ & $\begin{array}{r}1.43 \mathrm{E} 5 \\
1.49 \mathrm{E} 5\end{array}$ \\
\hline 442 & $\begin{array}{r}9.83 \mathrm{E} 6 \\
9.79 \mathrm{E} 6\end{array}$ & $\frac{2.74 \mathrm{E} 4}{3.64 \mathrm{E} 4}$ & $\begin{array}{r}1.62 \mathrm{E} 5 \\
1.74 \mathrm{E} 5\end{array}$ & $\frac{3.62 \mathrm{E} 6}{3.65 \mathrm{E} 6}$ & $\begin{array}{r}3.07 \mathrm{E} 6 \\
3.23 \mathrm{E} 6\end{array}$ & $\begin{array}{r}4.20 \mathrm{E} 7 \\
4.26 \mathrm{E} 7\end{array}$ & $\begin{array}{r}1.01 \mathrm{E} 5 \\
1.05 \mathrm{E} 5\end{array}$ \\
\hline 44 & $\frac{1.32 \mathrm{E} 7}{1.32 \mathrm{E} 7}$ & $\frac{3.10 \mathrm{E} 4}{3.30 \mathrm{E} 4}$ & $\frac{2.17 \mathrm{E} 5}{2.38 \mathrm{E} 5}$ & $\frac{5.14 \mathrm{E} 6}{5.24 \mathrm{E} 6}$ & $\frac{4.31 \mathrm{E} 6}{4.49 \mathrm{E} 6}$ & $5 \frac{5.66 \mathrm{E} 7}{5.72 \mathrm{E} 7}$ & $\frac{1.40 \mathrm{E} 5}{1.50 \mathrm{E} 5}$ \\
\hline 442 & $\begin{array}{r}1.29 \mathrm{E} 7 \\
1.28 \mathrm{E} 7\end{array}$ & $\frac{5.32 \mathrm{E} 4}{4.13 \mathrm{E} 4}$ & $\frac{2.07 \mathrm{E} 5}{2.26 \mathrm{E} 5}$ & $\frac{4.49 \mathrm{E} 6}{4.53 \mathrm{E} 6}$ & $\frac{3.99 \mathrm{E} 6}{4.12 \mathrm{E} 6}$ & $\frac{5.44 \mathrm{E} 7}{5.51 \mathrm{E} 7}$ & $\frac{1.28 \mathrm{E} 5}{1.35 \mathrm{E} 5}$ \\
\hline 442 & $\frac{1.11 \mathrm{E} 7}{1.10 \mathrm{E} 7}$ & $\frac{3.74 \mathrm{E} 4}{4.07 \mathrm{E} 4}$ & $\frac{1.84 \mathrm{E} 5}{2.00 \mathrm{E} 5}$ & $\frac{4.38 \mathrm{E} 6}{4.39 \mathrm{E} 6}$ & $\frac{3.63 \mathrm{E} 6}{3.76 \mathrm{E} 6}$ & $\frac{4.72 \mathrm{E} 7}{4.80 \mathrm{E} 7}$ & $\frac{1.21 \mathrm{E} 5}{1.28 \mathrm{E} 5}$ \\
\hline $\begin{array}{r}\mathrm{Av} \\
\mathrm{b}\end{array}$ & 0.999 & 0.949 & 0.918 & 0.986 & 0.958 & 0.987 & 0.954 \\
\hline bias & 0.6 & 20.2 & 1.7 & 0.6 & 0.7 & 0.5 & 1.5 \\
\hline
\end{tabular}

Note: Activity values in Bq/particle above the diagonal line were measured in 2014 at $35 \mathrm{~cm}$; values below the diagonal line were measured in 2011 at $50 \mathrm{~cm}$. Both values are decay-corrected back to 1 day after the end of irradiation. 
Table 3. Activity in Bq/particle of gamma-emitting isotopes in particles before safety test

\begin{tabular}{|c|c|c|c|c|c|c|c|}
\hline Particle & ${ }^{106} \mathrm{Ru}$ & ${ }^{110 m} \mathrm{Ag}$ & ${ }^{125} \mathrm{Sb}$ & ${ }^{134} \mathrm{Cs}$ & ${ }^{137} \mathrm{Cs}$ & ${ }^{144} \mathrm{Ce}$ & ${ }^{154} \mathbf{E u}$ \\
\hline 442-A001 & $1.29 \mathrm{E} 7$ & $4.45 \mathrm{E} 4$ & $2.12 \mathrm{E} 5$ & $25.01 \mathrm{E} 6$ & $4.16 \mathrm{E} 6$ & 5.30E7 & $1.41 \mathrm{E} 5$ \\
\hline 442-A002 & $1.36 \mathrm{E} 7$ & $5.05 \mathrm{E} 4$ & $2.18 \mathrm{E} 5$ & $5.39 \mathrm{E} 6$ & $4.32 \mathrm{E} 6$ & $5.35 \mathrm{E} 7$ & $1.47 \mathrm{E} 5$ \\
\hline 442-A003 & $1.44 \mathrm{E} 7$ & $4.75 \mathrm{E} 4$ & $2.30 \mathrm{E} 5$ & $4.83 \mathrm{E} 6$ & $4.25 \mathrm{E} 6$ & $5.91 \mathrm{E} 7$ & $1.35 \mathrm{E} 5$ \\
\hline 442-A004 & $1.38 \mathrm{E} 7$ & $4.68 \mathrm{E} 4$ & $2.11 \mathrm{E} 5$ & $4.92 \mathrm{E} 6$ & $4.10 \mathrm{E} 6$ & 5.39E7 & $1.36 \mathrm{E} 5$ \\
\hline 442-A005 & $1.15 \mathrm{E} 7$ & $1.81 \mathrm{E} 4$ & $1.90 \mathrm{E} 5$ & $4.34 \mathrm{E} 6$ & $3.67 \mathrm{E} 6$ & $4.85 \mathrm{E} 7$ & $1.21 \mathrm{E} 5$ \\
\hline 442-A006 & $1.39 \mathrm{E} 7$ & $5.31 \mathrm{E} 4$ & $2.22 \mathrm{E} 5$ & $5.40 \mathrm{E} 6$ & $4.38 \mathrm{E} 6$ & $5.48 \mathrm{E} 7$ & $1.47 \mathrm{E} 5$ \\
\hline 442-A007 & $1.18 \mathrm{E} 7$ & $2.81 \mathrm{E} 4$ & $1.95 \mathrm{E} 5$ & $4.45 \mathrm{E} 6$ & $3.83 \mathrm{E} 6$ & 5.07E7 & $1.27 \mathrm{E} 5$ \\
\hline 442-A008 & $1.22 \mathrm{E} 7$ & $4.15 \mathrm{E} 4$ & $1.98 \mathrm{E} 5$ & $4.72 \mathrm{E} 6$ & $3.94 \mathrm{E} 6$ & $5.08 \mathrm{E} 7$ & $1.31 \mathrm{E} 5$ \\
\hline 442-A009 & $1.35 \mathrm{E} 7$ & $2.55 \mathrm{E} 4$ & $2.16 \mathrm{E} 5$ & $5.06 \mathrm{E} 6$ & $4.22 \mathrm{E} 6$ & $5.52 \mathrm{E} 7$ & $1.39 \mathrm{E} 5$ \\
\hline 442-A010 & $1.26 \mathrm{E} 7$ & $4.06 \mathrm{E} 4$ & $2.00 \mathrm{E} 5$ & $4.26 \mathrm{E} 6$ & $3.84 \mathrm{E} 6$ & $5.27 \mathrm{E} 7$ & $1.25 \mathrm{E} 5$ \\
\hline 442-A011 & $1.21 \mathrm{E} 7$ & $3.91 \mathrm{E} 4$ & $2.01 \mathrm{E} 5$ & $4.46 \mathrm{E} 6$ & $3.85 \mathrm{E} 6$ & 5.07E7 & $1.28 \mathrm{E} 5$ \\
\hline 442-A012 & $1.20 \mathrm{E} 7$ & $3.54 \mathrm{E} 4$ & $2.03 \mathrm{E} 5$ & 4.49E6 & $3.86 \mathrm{E} 6$ & $5.14 \mathrm{E} 7$ & $1.26 \mathrm{E} 5$ \\
\hline 442-A013 & $1.50 \mathrm{E} 7$ & $5.65 \mathrm{E} 4$ & $2.35 \mathrm{E} 5$ & $5.87 \mathrm{E} 6$ & 4.64E6 & $5.54 \mathrm{E} 7$ & $1.66 \mathrm{E} 5$ \\
\hline 442-A014 & $1.25 \mathrm{E} 7$ & $4.46 \mathrm{E} 4$ & $2.11 \mathrm{E} 5$ & $4.83 \mathrm{E} 6$ & $4.02 \mathrm{E} 6$ & $5.15 \mathrm{E} 7$ & $1.33 \mathrm{E} 5$ \\
\hline 442-A015 & $1.31 \mathrm{E} 7$ & $3.71 \mathrm{E} 4$ & $2.09 \mathrm{E} 5$ & $4.98 \mathrm{E} 6$ & $4.16 \mathrm{E} 6$ & $5.48 \mathrm{E} 7$ & $1.35 \mathrm{E} 5$ \\
\hline 442-A016 & $1.18 \mathrm{E} 7$ & $3.58 \mathrm{E} 4$ & $1.92 \mathrm{E} 5$ & $4.31 \mathrm{E} 6$ & $3.68 \mathrm{E} 6$ & $4.90 \mathrm{E} 7$ & $1.23 \mathrm{E} 5$ \\
\hline 442-A017 & $1.36 \mathrm{E} 7$ & $4.12 \mathrm{E} 4$ & $2.21 \mathrm{E} 5$ & $4.79 \mathrm{E} 6$ & $4.21 \mathrm{E} 6$ & $5.66 \mathrm{E} 7$ & $1.38 \mathrm{E} 5$ \\
\hline 442-A018 & $1.28 \mathrm{E} 7$ & $4.47 \mathrm{E} 4$ & $2.05 \mathrm{E} 5$ & $4.83 \mathrm{E} 6$ & $3.99 \mathrm{E} 6$ & $5.08 \mathrm{E} 7$ & $1.35 \mathrm{E} 5$ \\
\hline 442-A020 & $1.46 \mathrm{E} 7$ & $4.88 \mathrm{E} 4$ & $2.33 \mathrm{E} 5$ & $5.53 \mathrm{E} 6$ & $4.59 \mathrm{E} 6$ & $5.67 \mathrm{E} 7$ & $1.61 \mathrm{E} 5$ \\
\hline 442-A022 & $1.41 \mathrm{E} 7$ & $4.74 \mathrm{E} 4$ & $2.21 \mathrm{E} 5$ & $4.79 \mathrm{E} 6$ & $4.15 \mathrm{E} 6$ & $5.56 \mathrm{E} 7$ & $1.39 \mathrm{E} 5$ \\
\hline 442-A023 & $1.28 \mathrm{E} 7$ & $3.86 \mathrm{E} 4$ & $2.04 \mathrm{E} 5$ & $4.40 \mathrm{E} 6$ & $3.94 \mathrm{E} 6$ & $5.42 \mathrm{E} 7$ & $1.26 \mathrm{E} 5$ \\
\hline 442-A024 & $1.34 \mathrm{E} 7$ & $3.28 \mathrm{E} 4$ & $2.20 \mathrm{E} 5$ & $4.99 \mathrm{E} 6$ & $4.21 \mathrm{E} 6$ & $5.55 \mathrm{E} 7$ & $1.37 \mathrm{E} 5$ \\
\hline 442-A026 & $1.70 \mathrm{E} 7$ & $5.99 \mathrm{E} 4$ & $2.74 \mathrm{E} 5$ & $6.83 \mathrm{E} 6$ & $5.49 \mathrm{E} 6$ & $6.54 \mathrm{E} 7$ & $1.93 \mathrm{E} 5$ \\
\hline 442-A027 & $1.31 \mathrm{E} 7$ & $4.34 \mathrm{E} 4$ & $2.06 \mathrm{E} 5$ & $4.43 \mathrm{E} 6$ & $3.88 \mathrm{E} 6$ & 5.19E7 & $1.28 \mathrm{E} 5$ \\
\hline 442-A028 & $1.44 \mathrm{E} 7$ & $4.79 \mathrm{E} 4$ & $2.30 \mathrm{E} 5$ & $4.66 \mathrm{E} 6$ & $4.20 \mathrm{E} 6$ & 5.87E7 & $1.36 \mathrm{E} 5$ \\
\hline 442-A029 & $1.40 \mathrm{E} 7$ & $4.93 \mathrm{E} 4$ & $2.24 \mathrm{E} 5$ & $5.08 \mathrm{E} 6$ & $4.31 \mathrm{E} 6$ & $5.58 \mathrm{E} 7$ & $1.48 \mathrm{E} 5$ \\
\hline 442-A030 & $1.29 \mathrm{E} 7$ & $4.10 \mathrm{E} 4$ & $2.10 \mathrm{E} 5$ & $4.64 \mathrm{E} 6$ & $4.11 \mathrm{E} 6$ & 5.57E7 & $1.33 \mathrm{E} 5$ \\
\hline 442-A031 & $1.24 \mathrm{E} 7$ & $4.08 \mathrm{E} 4$ & $1.97 \mathrm{E} 5$ & $4.16 \mathrm{E} 6$ & $3.72 \mathrm{E} 6$ & $5.12 \mathrm{E} 7$ & $1.21 \mathrm{E} 5$ \\
\hline 442-A032 & $1.29 \mathrm{E} 7$ & $4.86 \mathrm{E} 4$ & $2.13 \mathrm{E} 5$ & $5.28 \mathrm{E} 6$ & $4.21 \mathrm{E} 6$ & $5.23 \mathrm{E} 7$ & $1.44 \mathrm{E} 5$ \\
\hline 442-A033 & $1.07 \mathrm{E} 7$ & $3.25 \mathrm{E} 4$ & $1.78 \mathrm{E} 5$ & $4.04 \mathrm{E} 6$ & $3.38 \mathrm{E} 6$ & $4.41 \mathrm{E} 7$ & $1.14 \mathrm{E} 5$ \\
\hline 442-A034 & $1.26 \mathrm{E} 7$ & $4.31 \mathrm{E} 4$ & $2.07 \mathrm{E} 5$ & $5.03 \mathrm{E} 6$ & $4.05 \mathrm{E} 6$ & $4.95 \mathrm{E} 7$ & $1.41 \mathrm{E} 5$ \\
\hline 442-A037 & $1.19 \mathrm{E} 7$ & $3.73 \mathrm{E} 4$ & $1.94 \mathrm{E} 5$ & $4.37 \mathrm{E} 6$ & $3.68 \mathrm{E} 6$ & $4.76 \mathrm{E} 7$ & $1.28 \mathrm{E} 5$ \\
\hline 442-A038 & $1.19 \mathrm{E} 7$ & $3.53 \mathrm{E} 4$ & $1.94 \mathrm{E} 5$ & $3.86 \mathrm{E} 6$ & $3.51 \mathrm{E} 6$ & 4.93E7 & $1.14 \mathrm{E} 5$ \\
\hline 442-A040 & $1.42 \mathrm{E} 7$ & $5.10 \mathrm{E} 4$ & $2.19 \mathrm{E} 5$ & $4.93 \mathrm{E} 6$ & $4.13 \mathrm{E} 6$ & $5.44 \mathrm{E} 7$ & $1.40 \mathrm{E} 5$ \\
\hline 442-A041 & $1.16 \mathrm{E} 7$ & $3.90 \mathrm{E} 4$ & $1.89 \mathrm{E} 5$ & $4.16 \mathrm{E} 6$ & $3.67 \mathrm{E} 6$ & $4.98 \mathrm{E} 7$ & $1.20 \mathrm{E} 5$ \\
\hline 442-A042 & $1.27 \mathrm{E} 7$ & $4.55 \mathrm{E} 4$ & 2.07E5 & 4.47E6 & $3.92 \mathrm{E} 6$ & $5.20 \mathrm{E} 7$ & $1.29 \mathrm{E} 5$ \\
\hline 442-A043 & $1.39 \mathrm{E} 7$ & $4.91 \mathrm{E} 4$ & $2.32 \mathrm{E} 5$ & $5.30 \mathrm{E} 6$ & $4.45 \mathrm{E} 6$ & $5.70 \mathrm{E} 7$ & $1.49 \mathrm{E} 5$ \\
\hline 442-A044 & $1.35 \mathrm{E} 7$ & $4.22 \mathrm{E} 4$ & $2.28 \mathrm{E} 5$ & $5.38 \mathrm{E} 6$ & $4.39 \mathrm{E} 6$ & 5.61E7 & $1.46 \mathrm{E} 5$ \\
\hline 442-A045 & $1.32 \mathrm{E} 7$ & $4.87 \mathrm{E} 4$ & $2.14 \mathrm{E} 5$ & $5.05 \mathrm{E} 6$ & $4.07 \mathrm{E} 6$ & $5.10 \mathrm{E} 7$ & $1.41 \mathrm{E} 5$ \\
\hline 442-A046 & $1.05 \mathrm{E} 7$ & $4.18 \mathrm{E} 4$ & $1.65 \mathrm{E} 5$ & $4.17 \mathrm{E} 6$ & $3.24 \mathrm{E} 6$ & $3.99 \mathrm{E} 7$ & $1.15 \mathrm{E} 5$ \\
\hline 442-A047 & $1.06 \mathrm{E} 7$ & $2.66 \mathrm{E} 4$ & $1.76 \mathrm{E} 5$ & $3.97 \mathrm{E} 6$ & $3.37 \mathrm{E} 6$ & $4.54 \mathrm{E} 7$ & $1.11 \mathrm{E} 5$ \\
\hline
\end{tabular}


Table 3 (continued). Activity in Bq/particle of gamma-emitting isotopes in particles before safety test

\begin{tabular}{|c|c|c|c|c|c|c|c|}
\hline Particle & ${ }^{106} \mathrm{Ru}$ & ${ }^{110 \mathrm{~m}} \mathrm{Ag}$ & ${ }^{125} \mathrm{Sb}$ & ${ }^{134} \mathrm{Cs}$ & ${ }^{137} \mathrm{Cs}$ & ${ }^{144} \mathrm{Ce}$ & ${ }^{154} \mathbf{E u}$ \\
\hline 442-A048 & $1.33 \mathrm{E} 7$ & $4.59 \mathrm{E} 4$ & $2.12 \mathrm{E} 5$ & 4 4.87E6 & $4.14 \mathrm{E} 6$ & 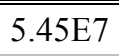 & $1.37 \mathrm{E} 5$ \\
\hline 442-A050 & $1.24 \mathrm{E} 7$ & $4.16 \mathrm{E} 4$ & $2.08 \mathrm{E} 5$ & $4.58 \mathrm{E} 6$ & $3.97 \mathrm{E} 6$ & 5.47E7 & $1.28 \mathrm{E} 5$ \\
\hline 442-A051 & $1.39 \mathrm{E} 7$ & $4.00 \mathrm{E} 4$ & $2.19 \mathrm{E} 5$ & $4.74 \mathrm{E} 6$ & $4.21 \mathrm{E} 6$ & $5.76 \mathrm{E} 7$ & $1.34 \mathrm{E} 5$ \\
\hline 442-A052 & $1.47 \mathrm{E} 7$ & $4.60 \mathrm{E} 4$ & $2.31 \mathrm{E} 5$ & $5.05 \mathrm{E} 6$ & $4.29 \mathrm{E} 6$ & $5.75 \mathrm{E} 7$ & $1.42 \mathrm{E} 5$ \\
\hline 442-A053 & $1.47 \mathrm{E} 7$ & $5.33 \mathrm{E} 4$ & $2.25 \mathrm{E} 5$ & $5.11 \mathrm{E} 6$ & $4.23 \mathrm{E} 6$ & $5.45 \mathrm{E} 7$ & $1.46 \mathrm{E} 5$ \\
\hline 442-A055 & $1.29 \mathrm{E} 7$ & $3.94 \mathrm{E} 4$ & $2.15 \mathrm{E} 5$ & 4.91E6 & $4.16 \mathrm{E} 6$ & $5.59 \mathrm{E} 7$ & $1.35 \mathrm{E} 5$ \\
\hline 442-A056 & $1.34 \mathrm{E} 7$ & $4.55 \mathrm{E} 4$ & $2.23 \mathrm{E} 5$ & 4.71E6 & $4.16 \mathrm{E} 6$ & 5.59E7 & $1.38 \mathrm{E} 5$ \\
\hline $442-\mathrm{A} 057$ & $1.34 \mathrm{E} 7$ & $4.63 \mathrm{E} 4$ & $2.14 \mathrm{E} 5$ & $5.12 \mathrm{E} 6$ & $4.09 \mathrm{E} 6$ & $5.10 \mathrm{E} 7$ & $1.42 \mathrm{E} 5$ \\
\hline 442-A058 & $1.28 \mathrm{E} 7$ & $4.59 \mathrm{E} 4$ & $2.16 \mathrm{E} 5$ & $5.00 \mathrm{E} 6$ & $4.19 \mathrm{E} 6$ & $5.39 \mathrm{E} 7$ & $1.38 \mathrm{E} 5$ \\
\hline 442-A059 & $1.41 \mathrm{E} 7$ & $4.14 \mathrm{E} 4$ & $2.37 \mathrm{E} 5$ & $5.38 \mathrm{E} 6$ & $4.57 \mathrm{E} 6$ & 5.93E7 & $1.50 \mathrm{E} 5$ \\
\hline $442-A 060$ & $1.19 \mathrm{E} 7$ & $4.77 \mathrm{E} 4$ & $1.92 \mathrm{E} 5$ & $4.78 \mathrm{E} 6$ & $3.84 \mathrm{E} 6$ & $4.86 \mathrm{E} 7$ & $1.31 \mathrm{E} 5$ \\
\hline 442-A061 & $1.27 \mathrm{E} 7$ & $4.34 \mathrm{E} 4$ & $2.04 \mathrm{E} 5$ & $4.79 \mathrm{E} 6$ & $3.97 \mathrm{E} 6$ & $5.02 \mathrm{E} 7$ & $1.35 \mathrm{E} 5$ \\
\hline 442-A062 & $1.40 \mathrm{E} 7$ & 4.93E4 & $2.23 \mathrm{E} 5$ & $5.27 \mathrm{E} 6$ & $4.28 \mathrm{E} 6$ & $5.45 \mathrm{E} 7$ & $1.47 \mathrm{E} 5$ \\
\hline 442-A063 & $9.78 \mathrm{E} 6$ & $3.46 \mathrm{E} 4$ & $1.60 \mathrm{E} 5$ & $3.60 \mathrm{E} 6$ & $3.10 \mathrm{E} 6$ & $4.21 \mathrm{E} 7$ & $1.00 \mathrm{E} 5$ \\
\hline 442-A064 & $1.24 \mathrm{E} 7$ & $3.71 \mathrm{E} 4$ & $1.96 \mathrm{E} 5$ & $4.33 \mathrm{E} 6$ & $3.77 \mathrm{E} 6$ & $5.10 \mathrm{E} 7$ & $1.26 \mathrm{E} 5$ \\
\hline 442-A065 & $1.47 \mathrm{E} 7$ & $5.62 \mathrm{E} 4$ & $2.30 \mathrm{E} 5$ & $5.11 \mathrm{E} 6$ & $4.34 \mathrm{E} 6$ & $5.74 \mathrm{E} 7$ & $1.45 \mathrm{E} 5$ \\
\hline 442-A067 & $1.29 \mathrm{E} 7$ & $4.92 \mathrm{E} 4$ & $2.02 \mathrm{E} 5$ & $5.16 \mathrm{E} 6$ & $4.12 \mathrm{E} 6$ & $5.09 \mathrm{E} 7$ & $1.43 \mathrm{E} 5$ \\
\hline 442-A068 & $1.30 \mathrm{E} 7$ & $3.40 \mathrm{E} 4$ & $2.24 \mathrm{E} 5$ & $5.11 \mathrm{E} 6$ & 4.34E6 & $5.59 \mathrm{E} 7$ & $1.43 \mathrm{E} 5$ \\
\hline 442-A070 & $1.29 \mathrm{E} 7$ & $4.68 \mathrm{E} 4$ & $2.10 \mathrm{E} 5$ & $5.12 \mathrm{E} 6$ & $4.11 \mathrm{E} 6$ & 5.17E7 & $1.41 \mathrm{E} 5$ \\
\hline 442-A071 & $1.36 \mathrm{E} 7$ & $4.24 \mathrm{E} 4$ & $2.17 \mathrm{E} 5$ & $4.77 \mathrm{E} 6$ & $4.11 \mathrm{E} 6$ & $5.56 \mathrm{E} 7$ & $1.34 \mathrm{E} 5$ \\
\hline 442-A072 & $1.32 \mathrm{E} 7$ & $3.13 \mathrm{E} 4$ & $2.18 \mathrm{E} 5$ & $5.16 \mathrm{E} 6$ & $4.30 \mathrm{E} 6$ & $5.64 \mathrm{E} 7$ & $1.43 \mathrm{E} 5$ \\
\hline $442-\mathrm{A} 073$ & $1.44 \mathrm{E} 7$ & $4.92 \mathrm{E} 4$ & $2.21 \mathrm{E} 5$ & 4.97E6 & $4.12 \mathrm{E} 6$ & $5.20 \mathrm{E} 7$ & $1.44 \mathrm{E} 5$ \\
\hline 442-A074 & $1.23 \mathrm{E} 7$ & $4.27 \mathrm{E} 4$ & $1.90 \mathrm{E} 5$ & $4.28 \mathrm{E} 6$ & $3.62 \mathrm{E} 6$ & $4.82 \mathrm{E} 7$ & $1.18 \mathrm{E} 5$ \\
\hline 442-A075 & $1.22 \mathrm{E} 7$ & $3.79 \mathrm{E} 4$ & $1.89 \mathrm{E} 5$ & $4.03 \mathrm{E} 6$ & $3.56 \mathrm{E} 6$ & $4.91 \mathrm{E} 7$ & $1.15 \mathrm{E} 5$ \\
\hline 442-A076 & $1.26 \mathrm{E} 7$ & 4.63E4 & $2.05 \mathrm{E} 5$ & 5.03E6 & $4.06 \mathrm{E} 6$ & 5.09E7 & $1.37 \mathrm{E} 5$ \\
\hline 442-A077 & $1.33 \mathrm{E} 7$ & $4.33 \mathrm{E} 4$ & $2.04 \mathrm{E} 5$ & $4.36 \mathrm{E} 6$ & $3.84 \mathrm{E} 6$ & 5.32E7 & $1.23 \mathrm{E} 5$ \\
\hline 442-A078 & $1.28 \mathrm{E} 7$ & $3.92 \mathrm{E} 4$ & $2.08 \mathrm{E} 5$ & $4.46 \mathrm{E} 6$ & $3.95 \mathrm{E} 6$ & $5.44 \mathrm{E} 7$ & $1.28 \mathrm{E} 5$ \\
\hline 442-A079 & $1.26 \mathrm{E} 7$ & $4.56 \mathrm{E} 4$ & $2.01 \mathrm{E} 5$ & $4.51 \mathrm{E} 6$ & $3.87 \mathrm{E} 6$ & $5.12 \mathrm{E} 7$ & $1.29 \mathrm{E} 5$ \\
\hline 442-A081 & $1.23 \mathrm{E} 7$ & $4.27 \mathrm{E} 4$ & $1.98 \mathrm{E} 5$ & 4.54E6 & $3.87 \mathrm{E} 6$ & 5.17E7 & $1.27 \mathrm{E} 5$ \\
\hline 442-A082 & $1.35 \mathrm{E} 7$ & $4.78 \mathrm{E} 4$ & $2.25 \mathrm{E} 5$ & $4.58 \mathrm{E} 6$ & $4.10 \mathrm{E} 6$ & $5.64 \mathrm{E} 7$ & $1.31 \mathrm{E} 5$ \\
\hline 442-A083 & $1.33 \mathrm{E} 7$ & $4.45 \mathrm{E} 4$ & $2.07 \mathrm{E} 5$ & 4.67E6 & $3.92 \mathrm{E} 6$ & $5.08 \mathrm{E} 7$ & $1.33 \mathrm{E} 5$ \\
\hline 442-A084 & $1.31 \mathrm{E} 7$ & $4.53 \mathrm{E} 4$ & $2.06 \mathrm{E} 5$ & 4.49E6 & $3.92 \mathrm{E} 6$ & $5.30 \mathrm{E} 7$ & $1.28 \mathrm{E} 5$ \\
\hline 442-A085 & $1.19 \mathrm{E} 7$ & $4.19 \mathrm{E} 4$ & $1.94 \mathrm{E} 5$ & 4.79E6 & $3.89 \mathrm{E} 6$ & 4.92E7 & $1.32 \mathrm{E} 5$ \\
\hline 442-A086 & $1.30 \mathrm{E} 7$ & $3.92 \mathrm{E} 4$ & $2.08 \mathrm{E} 5$ & $4.36 \mathrm{E} 6$ & $3.92 \mathrm{E} 6$ & $5.44 \mathrm{E} 7$ & $1.28 \mathrm{E} 5$ \\
\hline Maximum & $1.70 \mathrm{E} 7$ & $5.99 \mathrm{E} 4$ & $2.74 \mathrm{E} 5$ & $6.83 \mathrm{E} 6$ & $5.49 \mathrm{E} 6$ & $6.54 \mathrm{E} 7$ & $1.93 \mathrm{E} 5$ \\
\hline Minimum & $9.78 \mathrm{E} 6$ & $1.81 \mathrm{E} 4$ & $1.60 \mathrm{E} 5$ & $3.60 \mathrm{E} 6$ & $3.10 \mathrm{E} 6$ & 3.99E7 & $1.00 \mathrm{E} 5$ \\
\hline Average & $1.30 \mathrm{E} 7$ & $4.27 \mathrm{E} 4$ & $2.10 \mathrm{E} 5$ & $4.78 \mathrm{E} 6$ & 4.04E6 & $5.29 \mathrm{E} 7$ & $1.35 \mathrm{E} 5$ \\
\hline$\%$ SD & 9 & 17 & 8 & 10 & 8 & 8 & 10 \\
\hline
\end{tabular}

Note: Values are decay-corrected back to 1 day after the end of irradiation and pre-test values are adjusted using average bias in Table 2 to account for different counting geometry. 
After completion of the CCCTF test, particles were removed from the graphite tray assembly and returned to their original vials for six-hour IMGA counting at a $35 \mathrm{~cm}$ detector geometry. The results of the postsafety test IMGA measurements are given in Table 4, and Table 5 shows the calculated ratio of the activity measured after the safety test divided by the activity before the safety test (i.e., the fraction of each isotope retained in each particle); data in Table 5 are also presented graphically in Figure 10. Five particles (highlighted in gray in Table 4 and Table 5) exhibited 0-1\% Cs retention; this is consistent with the safety test results (Figure 2), where cesium and krypton release indicated the presence of five particles with failed TRISO. Ruthenium retention did not appear to be affected by the TRISO failure in these five particles and was uniformly high in the entire sample. Retention of the other monitored radioisotopes in the five particles with failed TRISO varied; this is discussed in conjunction with the microstructural analysis in the next section.

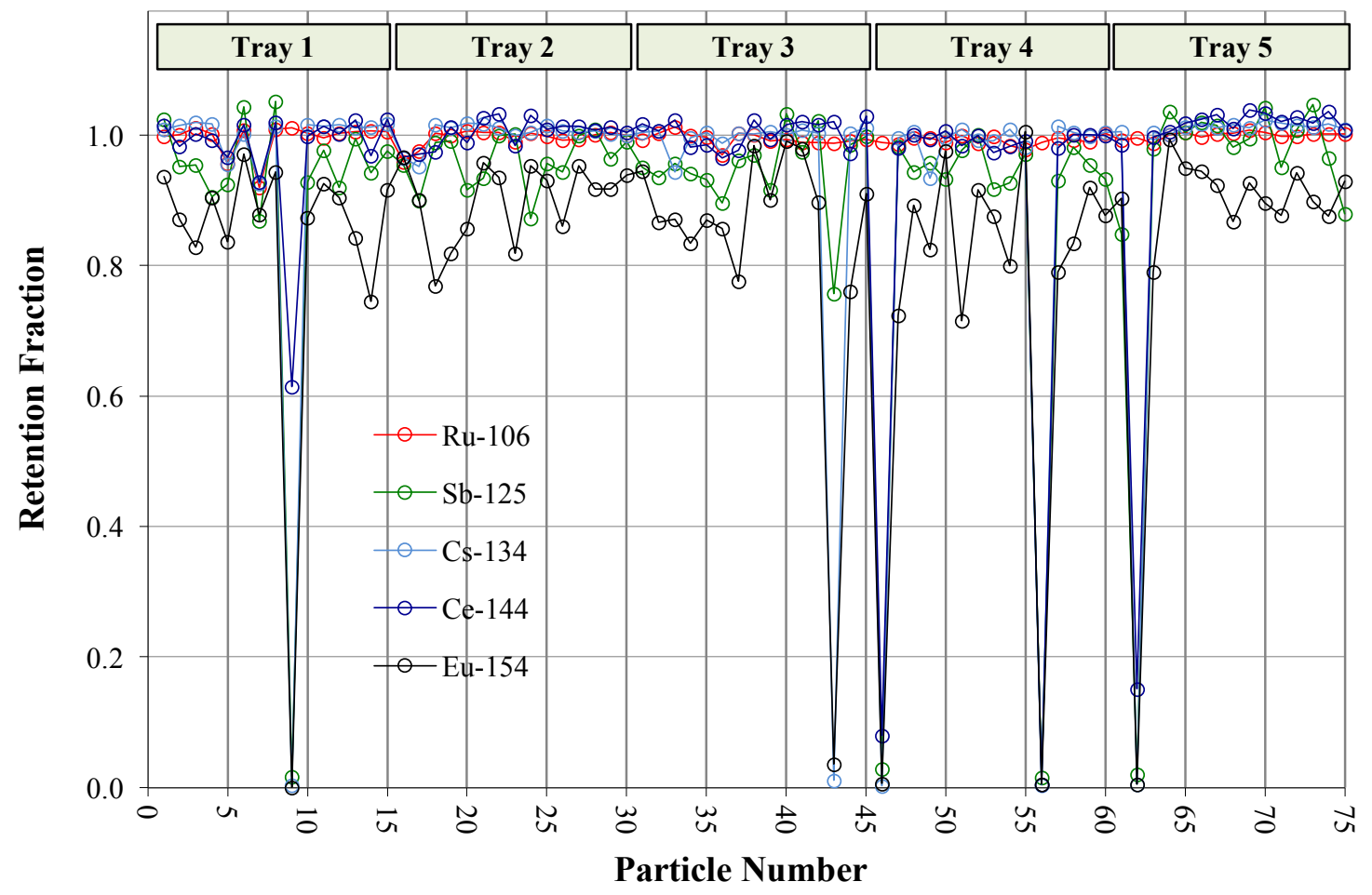

Figure 10. Radioisotope retention fraction of 75 particles after $1800^{\circ} \mathrm{C}$ safety testing.

Not counting the five abnormal particles, there was no measurable release of ${ }^{106} \mathrm{Ru},{ }^{134} \mathrm{Cs},{ }^{137} \mathrm{Cs}$, or ${ }^{144} \mathrm{Ce}$ from the remaining seventy particles; the average retention fraction for these isotopes was close to $100 \%$. The average retention fraction for ${ }^{125} \mathrm{Sb}$ was $96 \%$, but this deviation from $100 \%$ may not be significant compared to the measurement uncertainty. However, the average retention fraction for ${ }^{154} \mathrm{Eu}$ of $89 \%$ indicates a measurable release of europium from the particles. Comparison of the ${ }^{110 \mathrm{~m}} \mathrm{Ag}$ activity in each particle before and after the safety test clearly shows that silver was also released from the particles during the $1800^{\circ} \mathrm{C}$ safety test, as expected based on the silver release observed in the CCCTF (Figure 4). The remaining ${ }^{110 \mathrm{~m}} \mathrm{Ag}$ activity in most of the particles was too low to measure with IMGA; for these particles the estimated ${ }^{110 \mathrm{~m}} \mathrm{Ag}$ activity quantification limit and retention fraction are given as less-than values in Table 4 and Table 5. The overall low ${ }^{110 \mathrm{~m}} \mathrm{Ag}$ inventory after the safety test agrees with the indication in the CCCTF time-dependent ${ }^{110 \mathrm{~m}} \mathrm{Ag}$ release data that the ${ }^{110 \mathrm{~m}} \mathrm{Ag}$ inventory was becoming depleted by the end of the safety test. Only six particles retained enough silver to measure the ${ }^{110 \mathrm{~m}} \mathrm{Ag}$ activity with IMGA using a six-hour counting time. At least one of these particles, Particle 442-A074, had $a^{110 \mathrm{~m}} \mathrm{Ag}$ inventory that indicated high retention, in contrast to most of the particles in the test. 
Table 4. Activity in Bq/particle of gamma-emitting isotopes in particles after safety test

\begin{tabular}{|c|c|c|c|c|c|c|c|}
\hline Particle & ${ }^{106} \mathrm{Ru}$ & ${ }^{110 \mathrm{~m}} \mathrm{Ag}$ & ${ }^{125} \mathrm{Sb}$ & ${ }^{134} \mathrm{Cs}$ & ${ }^{137} \mathrm{Cs}$ & ${ }^{144} \mathrm{Ce}$ & ${ }^{154} \mathbf{E u}$ \\
\hline 442-A001 & $1.29 \mathrm{E} 7$ & $<<1.59 \mathrm{E} 4$ & $2.17 \mathrm{E} 5$ & $25.05 \mathrm{E} 6$ & $4.21 \mathrm{E} 6$ & $\begin{array}{l}5.38 \mathrm{E} 7 \\
\end{array}$ & $1.32 \mathrm{E} 5$ \\
\hline 442-A002 & $1.36 \mathrm{E} 7$ & $<1.64 \mathrm{E} 4$ & $2.08 \mathrm{E} 5$ & $5.47 \mathrm{E} 6$ & $4.38 \mathrm{E} 6$ & $5.26 \mathrm{E} 7$ & $1.28 \mathrm{E} 5$ \\
\hline 442-A003 & $1.45 \mathrm{E} 7$ & $<1.57 \mathrm{E} 4$ & $2.20 \mathrm{E} 5$ & $4.93 \mathrm{E} 6$ & $4.34 \mathrm{E} 6$ & 5.92E7 & $1.12 \mathrm{E} 5$ \\
\hline 442-A004 & $1.39 \mathrm{E} 7$ & $<1.60 \mathrm{E} 4$ & 1.91E5 & $5.00 \mathrm{E} 6$ & $4.17 \mathrm{E} 6$ & $5.36 \mathrm{E} 7$ & $1.23 \mathrm{E} 5$ \\
\hline 442-A005 & $1.10 \mathrm{E} 7$ & $<1.46 \mathrm{E} 4$ & $1.75 \mathrm{E} 5$ & $4.15 \mathrm{E} 6$ & $3.54 \mathrm{E} 6$ & 4.69E7 & $1.01 \mathrm{E} 5$ \\
\hline 442-A006 & $1.41 \mathrm{E} 7$ & $<9.67 \mathrm{E} 3$ & $2.32 \mathrm{E} 5$ & $5.41 \mathrm{E} 6$ & $4.40 \mathrm{E} 6$ & 5.57E7 & $1.43 \mathrm{E} 5$ \\
\hline 442-A007 & $1.09 \mathrm{E} 7$ & $<1.44 \mathrm{E} 4$ & 1.69E5 & $4.12 \mathrm{E} 6$ & $3.59 \mathrm{E} 6$ & $4.70 \mathrm{E} 7$ & $1.11 \mathrm{E} 5$ \\
\hline 442-A008 & $1.23 \mathrm{E} 7$ & $<1.51 \mathrm{E} 4$ & $2.08 \mathrm{E} 5$ & $4.78 \mathrm{E} 6$ & $4.03 \mathrm{E} 6$ & $5.18 \mathrm{E} 7$ & $1.23 \mathrm{E} 5$ \\
\hline 442-A009 & $1.36 \mathrm{E} 7$ & $<7.02 \mathrm{E} 3$ & $3.68 \mathrm{E} 3$ & $1.57 \mathrm{E} 4$ & $2.46 \mathrm{E} 4$ & $3.39 \mathrm{E} 7$ & $3.87 \mathrm{E} 1$ \\
\hline 442-A010 & $1.27 \mathrm{E} 7$ & $<1.46 \mathrm{E} 4$ & $1.86 \mathrm{E} 5$ & $4.34 \mathrm{E} 6$ & $3.90 \mathrm{E} 6$ & $5.26 \mathrm{E} 7$ & $1.09 \mathrm{E} 5$ \\
\hline 442-A011 & $1.20 \mathrm{E} 7$ & $<1.51 \mathrm{E} 4$ & $1.96 \mathrm{E} 5$ & $4.52 \mathrm{E} 6$ & $3.90 \mathrm{E} 6$ & $5.13 \mathrm{E} 7$ & $1.18 \mathrm{E} 5$ \\
\hline 442-A012 & $1.21 \mathrm{E} 7$ & $<1.47 \mathrm{E} 4$ & $1.87 \mathrm{E} 5$ & $4.57 \mathrm{E} 6$ & $3.93 \mathrm{E} 6$ & $5.15 \mathrm{E} 7$ & $1.14 \mathrm{E} 5$ \\
\hline 442-A013 & $1.50 \mathrm{E} 7$ & $<1.67 \mathrm{E} 4$ & $2.34 \mathrm{E} 5$ & $5.93 \mathrm{E} 6$ & $4.66 \mathrm{E} 6$ & 5.67E7 & $1.40 \mathrm{E} 5$ \\
\hline 442-A014 & $1.25 \mathrm{E} 7$ & $<1.50 \mathrm{E} 4$ & 1.99E5 & $4.89 \mathrm{E} 6$ & 4.07E6 & 4.99E7 & $9.90 \mathrm{E} 4$ \\
\hline 442-A015 & $1.32 \mathrm{E} 7$ & $<1.58 \mathrm{E} 4$ & $2.04 \mathrm{E} 5$ & $5.06 \mathrm{E} 6$ & $4.23 \mathrm{E} 6$ & $5.62 \mathrm{E} 7$ & $1.24 \mathrm{E} 5$ \\
\hline 442-A016 & $1.13 \mathrm{E} 7$ & $<1.58 \mathrm{E} 4$ & $1.83 \mathrm{E} 5$ & $4.17 \mathrm{E} 6$ & $3.55 \mathrm{E} 6$ & $4.73 \mathrm{E} 7$ & $1.19 \mathrm{E} 5$ \\
\hline 442-A017 & $1.32 \mathrm{E} 7$ & $<1.65 \mathrm{E} 4$ & $1.99 \mathrm{E} 5$ & $4.56 \mathrm{E} 6$ & $4.13 \mathrm{E} 6$ & 5.49E7 & $1.25 \mathrm{E} 5$ \\
\hline 442-A018 & $1.28 \mathrm{E} 7$ & $<1.62 \mathrm{E} 4$ & 2.03E5 & $4.91 \mathrm{E} 6$ & $4.02 \mathrm{E} 6$ & $4.95 \mathrm{E} 7$ & $1.04 \mathrm{E} 5$ \\
\hline 442-A020 & $1.46 \mathrm{E} 7$ & $<1.78 \mathrm{E} 4$ & $2.31 \mathrm{E} 5$ & $5.59 \mathrm{E} 6$ & $4.66 \mathrm{E} 6$ & $5.74 \mathrm{E} 7$ & $1.32 \mathrm{E} 5$ \\
\hline 442-A022 & $1.42 \mathrm{E} 7$ & $<1.64 \mathrm{E} 4$ & $2.03 \mathrm{E} 5$ & $4.88 \mathrm{E} 6$ & $4.20 \mathrm{E} 6$ & $5.50 \mathrm{E} 7$ & $1.19 \mathrm{E} 5$ \\
\hline 442-A023 & $1.29 \mathrm{E} 7$ & $<1.57 \mathrm{E} 4$ & $1.91 \mathrm{E} 5$ & $4.47 \mathrm{E} 6$ & $4.02 \mathrm{E} 6$ & 5.57E7 & $1.21 \mathrm{E} 5$ \\
\hline 442-A024 & $1.35 \mathrm{E} 7$ & $<1.67 \mathrm{E} 4$ & $2.20 \mathrm{E} 5$ & $5.05 \mathrm{E} 6$ & $4.27 \mathrm{E} 6$ & $5.74 \mathrm{E} 7$ & $1.28 \mathrm{E} 5$ \\
\hline 442-A026 & $1.68 \mathrm{E} 7$ & $<1.95 \mathrm{E} 4$ & $2.75 \mathrm{E} 5$ & $6.83 \mathrm{E} 6$ & $5.55 \mathrm{E} 6$ & $6.44 \mathrm{E} 7$ & $1.58 \mathrm{E} 5$ \\
\hline 442-A027 & $1.31 \mathrm{E} 7$ & $<1.45 \mathrm{E} 4$ & $1.80 \mathrm{E} 5$ & $4.45 \mathrm{E} 6$ & $3.90 \mathrm{E} 6$ & $5.35 \mathrm{E} 7$ & $1.22 \mathrm{E} 5$ \\
\hline 442-A028 & $1.44 \mathrm{E} 7$ & $<1.50 \mathrm{E} 4$ & $2.20 \mathrm{E} 5$ & $4.73 \mathrm{E} 6$ & $4.22 \mathrm{E} 6$ & $5.92 \mathrm{E} 7$ & $1.26 \mathrm{E} 5$ \\
\hline 442-A029 & $1.39 \mathrm{E} 7$ & $<1.55 \mathrm{E} 4$ & $2.11 \mathrm{E} 5$ & $5.12 \mathrm{E} 6$ & $4.34 \mathrm{E} 6$ & $5.66 \mathrm{E} 7$ & $1.27 \mathrm{E} 5$ \\
\hline 442-A030 & $1.28 \mathrm{E} 7$ & $2.57 \mathrm{E} 4$ & $2.10 \mathrm{E} 5$ & $4.66 \mathrm{E} 6$ & $4.14 \mathrm{E} 6$ & $5.65 \mathrm{E} 7$ & $1.27 \mathrm{E} 5$ \\
\hline 442-A031 & $1.24 \mathrm{E} 7$ & $<1.44 \mathrm{E} 4$ & $1.99 \mathrm{E} 5$ & $4.18 \mathrm{E} 6$ & $3.76 \mathrm{E} 6$ & 5.17E7 & $1.11 \mathrm{E} 5$ \\
\hline 442-A032 & $1.30 \mathrm{E} 7$ & $<1.58 \mathrm{E} 4$ & $2.05 \mathrm{E} 5$ & $5.29 \mathrm{E} 6$ & $4.28 \mathrm{E} 6$ & $5.30 \mathrm{E} 7$ & $1.32 \mathrm{E} 5$ \\
\hline 442-A033 & $1.07 \mathrm{E} 7$ & $<1.38 \mathrm{E} 4$ & $1.76 \mathrm{E} 5$ & $4.04 \mathrm{E} 6$ & $3.42 \mathrm{E} 6$ & $4.42 \mathrm{E} 7$ & $1.07 \mathrm{E} 5$ \\
\hline 442-A034 & $1.25 \mathrm{E} 7$ & $<1.54 \mathrm{E} 4$ & $1.96 \mathrm{E} 5$ & $5.06 \mathrm{E} 6$ & $4.08 \mathrm{E} 6$ & 5.03E7 & $1.33 \mathrm{E} 5$ \\
\hline 442-A037 & $1.19 \mathrm{E} 7$ & $<1.42 \mathrm{E} 4$ & $1.82 \mathrm{E} 5$ & $4.40 \mathrm{E} 6$ & $3.72 \mathrm{E} 6$ & 4.79E7 & $1.11 \mathrm{E} 5$ \\
\hline 442-A038 & $1.20 \mathrm{E} 7$ & $<1.38 \mathrm{E} 4$ & $1.85 \mathrm{E} 5$ & $3.64 \mathrm{E} 6$ & $3.55 \mathrm{E} 6$ & $5.04 \mathrm{E} 7$ & $9.92 \mathrm{E} 4$ \\
\hline 442-A040 & $1.42 \mathrm{E} 7$ & $<1.49 \mathrm{E} 4$ & $2.06 \mathrm{E} 5$ & $4.91 \mathrm{E} 6$ & $4.14 \mathrm{E} 6$ & $5.34 \mathrm{E} 7$ & $1.17 \mathrm{E} 5$ \\
\hline 442-A041 & $1.16 \mathrm{E} 7$ & $<1.38 \mathrm{E} 4$ & $1.76 \mathrm{E} 5$ & $4.17 \mathrm{E} 6$ & $3.68 \mathrm{E} 6$ & $4.91 \mathrm{E} 7$ & $1.05 \mathrm{E} 5$ \\
\hline 442-A042 & $1.23 \mathrm{E} 7$ & $<1.40 \mathrm{E} 4$ & $1.85 \mathrm{E} 5$ & $4.42 \mathrm{E} 6$ & $3.80 \mathrm{E} 6$ & $5.02 \mathrm{E} 7$ & $1.11 \mathrm{E} 5$ \\
\hline 442-A043 & $1.40 \mathrm{E} 7$ & $<1.55 \mathrm{E} 4$ & $2.23 \mathrm{E} 5$ & $5.32 \mathrm{E} 6$ & $4.51 \mathrm{E} 6$ & 5.57E7 & $1.16 \mathrm{E} 5$ \\
\hline 442-A044 & $1.35 \mathrm{E} 7$ & $2.08 \mathrm{E} 4$ & $2.21 \mathrm{E} 5$ & $5.39 \mathrm{E} 6$ & $4.46 \mathrm{E} 6$ & $5.74 \mathrm{E} 7$ & $1.44 \mathrm{E} 5$ \\
\hline 442-A045 & $1.31 \mathrm{E} 7$ & $<1.53 \mathrm{E} 4$ & $1.96 \mathrm{E} 5$ & $5.08 \mathrm{E} 6$ & $4.10 \mathrm{E} 6$ & 5.07E7 & $1.27 \mathrm{E} 5$ \\
\hline 442-A046 & $1.05 \mathrm{E} 7$ & $3.54 \mathrm{E} 4$ & $1.71 \mathrm{E} 5$ & $4.17 \mathrm{E} 6$ & $3.27 \mathrm{E} 6$ & $4.05 \mathrm{E} 7$ & $1.14 \mathrm{E} 5$ \\
\hline 442-A047 & $1.05 \mathrm{E} 7$ & $<1.39 \mathrm{E} 4$ & 1.71E5 & $4.00 \mathrm{E} 6$ & $3.40 \mathrm{E} 6$ & 4.64E7 & $1.08 \mathrm{E} 5$ \\
\hline
\end{tabular}


Table 4 (continued). Activity in Bq/particle of gamma-emitting isotopes in particles after safety test

\begin{tabular}{|c|c|c|c|c|c|c|c|}
\hline Particle & ${ }^{106} \mathrm{Ru}$ & ${ }^{110 m} \mathrm{Ag}$ & ${ }^{125} \mathrm{Sb}$ & ${ }^{134} \mathrm{Cs}$ & ${ }^{137} \mathrm{Cs}$ & ${ }^{144} \mathrm{Ce}$ & ${ }^{154} \mathrm{Eu}$ \\
\hline 442-A048 & $1.31 \mathrm{E} 7$ & $<<1.53 \mathrm{E} 4$ & $2.17 \mathrm{E} 5$ & $4.88 \mathrm{E} 6$ & 4.19E6 & $\begin{array}{c}5.54 \mathrm{E} 7 \\
\end{array}$ & $1.23 \mathrm{E} 5$ \\
\hline 442-A050 & $1.22 \mathrm{E} 7$ & $<7.40 \mathrm{E} 3$ & $1.57 \mathrm{E} 5$ & $4.97 \mathrm{E} 4$ & $5.48 \mathrm{E} 4$ & $5.58 \mathrm{E} 7$ & $4.60 \mathrm{E} 3$ \\
\hline 442-A051 & $1.38 \mathrm{E} 7$ & $<1.50 \mathrm{E} 4$ & $2.15 \mathrm{E} 5$ & $4.75 \mathrm{E} 6$ & $4.23 \mathrm{E} 6$ & $5.60 \mathrm{E} 7$ & $1.02 \mathrm{E} 5$ \\
\hline 442-A052 & $1.47 \mathrm{E} 7$ & $<1.58 \mathrm{E} 4$ & $2.31 \mathrm{E} 5$ & $5.11 \mathrm{E} 6$ & 4.35E6 & $5.93 \mathrm{E} 7$ & $1.30 \mathrm{E} 5$ \\
\hline 442-A053 & $1.45 \mathrm{E} 7$ & $<6.37 \mathrm{E} 3$ & $6.36 \mathrm{E} 3$ & $1.09 \mathrm{E} 4$ & $2.21 \mathrm{E} 4$ & $4.31 \mathrm{E} 6$ & $8.75 \mathrm{E} 2$ \\
\hline 442-A055 & $1.28 \mathrm{E} 7$ & $<1.46 \mathrm{E} 4$ & $2.11 \mathrm{E} 5$ & $4.89 \mathrm{E} 6$ & 4.19E6 & $5.48 \mathrm{E} 7$ & $9.76 \mathrm{E} 4$ \\
\hline 442-A056 & $1.34 \mathrm{E} 7$ & $1.39 \mathrm{E} 4$ & $2.11 \mathrm{E} 5$ & 4.73E6 & $4.18 \mathrm{E} 6$ & $5.59 \mathrm{E} 7$ & $1.23 \mathrm{E} 5$ \\
\hline 442-A057 & $1.34 \mathrm{E} 7$ & $<1.54 \mathrm{E} 4$ & $2.05 \mathrm{E} 5$ & $4.78 \mathrm{E} 6$ & $4.14 \mathrm{E} 6$ & $5.07 \mathrm{E} 7$ & $1.17 \mathrm{E} 5$ \\
\hline $442-\mathrm{A} 058$ & $1.27 \mathrm{E} 7$ & $<1.57 \mathrm{E} 4$ & $2.02 \mathrm{E} 5$ & $4.98 \mathrm{E} 6$ & $4.21 \mathrm{E} 6$ & $5.43 \mathrm{E} 7$ & $1.35 \mathrm{E} 5$ \\
\hline 442-A059 & $1.41 \mathrm{E} 7$ & $<1.55 \mathrm{E} 4$ & $2.32 \mathrm{E} 5$ & $5.43 \mathrm{E} 6$ & 4.63E6 & $5.83 \mathrm{E} 7$ & $1.07 \mathrm{E} 5$ \\
\hline 442-A060 & $1.17 \mathrm{E} 7$ & $<1.49 \mathrm{E} 4$ & $1.92 \mathrm{E} 5$ & $4.75 \mathrm{E} 6$ & $3.84 \mathrm{E} 6$ & $4.86 \mathrm{E} 7$ & $1.20 \mathrm{E} 5$ \\
\hline 442-A061 & $1.27 \mathrm{E} 7$ & $<1.47 \mathrm{E} 4$ & $1.87 \mathrm{E} 5$ & $4.75 \mathrm{E} 6$ & $4.00 \mathrm{E} 6$ & $4.88 \mathrm{E} 7$ & $1.18 \mathrm{E} 5$ \\
\hline 442-A062 & $1.38 \mathrm{E} 7$ & $<1.52 \mathrm{E} 4$ & 2.07E5 & $5.32 \mathrm{E} 6$ & $4.29 \mathrm{E} 6$ & $5.36 \mathrm{E} 7$ & $1.18 \mathrm{E} 5$ \\
\hline $442-A 063$ & $9.57 \mathrm{E} 6$ & $1.74 \mathrm{E} 4$ & $1.55 \mathrm{E} 5$ & $3.53 \mathrm{E} 6$ & $3.08 \mathrm{E} 6$ & 4.17E7 & $1.01 \mathrm{E} 5$ \\
\hline 442-A064 & $1.22 \mathrm{E} 7$ & $<6.27 \mathrm{E} 3$ & $3.09 \mathrm{E} 3$ & $1.34 \mathrm{E} 4$ & $2.49 \mathrm{E} 4$ & $2.69 \mathrm{E} 5$ & $5.45 \mathrm{E} 2$ \\
\hline 442-A065 & $1.46 \mathrm{E} 7$ & $<1.53 \mathrm{E} 4$ & $2.14 \mathrm{E} 5$ & $5.19 \mathrm{E} 6$ & 4.37E6 & $5.63 \mathrm{E} 7$ & $1.15 \mathrm{E} 5$ \\
\hline 442-A067 & $1.28 \mathrm{E} 7$ & $<1.46 \mathrm{E} 4$ & $1.98 \mathrm{E} 5$ & $5.18 \mathrm{E} 6$ & $4.14 \mathrm{E} 6$ & $5.09 \mathrm{E} 7$ & $1.19 \mathrm{E} 5$ \\
\hline 442-A068 & $1.29 \mathrm{E} 7$ & $<1.48 \mathrm{E} 4$ & $2.14 \mathrm{E} 5$ & $5.10 \mathrm{E} 6$ & $4.32 \mathrm{E} 6$ & $5.60 \mathrm{E} 7$ & $1.31 \mathrm{E} 5$ \\
\hline 442-A070 & $1.29 \mathrm{E} 7$ & $<1.50 \mathrm{E} 4$ & $1.96 \mathrm{E} 5$ & $5.14 \mathrm{E} 6$ & $4.16 \mathrm{E} 6$ & $5.16 \mathrm{E} 7$ & $1.23 \mathrm{E} 5$ \\
\hline 442-A071 & $1.35 \mathrm{E} 7$ & $<1.45 \mathrm{E} 4$ & $1.85 \mathrm{E} 5$ & $4.80 \mathrm{E} 6$ & $4.11 \mathrm{E} 6$ & $5.47 \mathrm{E} 7$ & $1.21 \mathrm{E} 5$ \\
\hline 442-A072 & $1.31 \mathrm{E} 7$ & $<6.56 \mathrm{E} 3$ & $4.41 \mathrm{E} 3$ & $2.61 \mathrm{E} 4$ & $3.26 \mathrm{E} 4$ & $8.50 \mathrm{E} 6$ & $7.50 \mathrm{E} 2$ \\
\hline 442-A073 & $1.42 \mathrm{E} 7$ & $<1.48 \mathrm{E} 4$ & $2.16 \mathrm{E} 5$ & $5.00 \mathrm{E} 6$ & 4.13E6 & $5.19 \mathrm{E} 7$ & $1.14 \mathrm{E} 5$ \\
\hline 442-A074 & $1.23 \mathrm{E} 7$ & $4.65 \mathrm{E} 4$ & $1.97 \mathrm{E} 5$ & $4.28 \mathrm{E} 6$ & 3.64E6 & $4.85 \mathrm{E} 7$ & $1.17 \mathrm{E} 5$ \\
\hline 442-A075 & $1.23 \mathrm{E} 7$ & $<1.42 \mathrm{E} 4$ & $1.90 \mathrm{E} 5$ & $4.08 \mathrm{E} 6$ & $3.57 \mathrm{E} 6$ & $5.00 \mathrm{E} 7$ & $1.09 \mathrm{E} 5$ \\
\hline 442-A076 & $1.25 \mathrm{E} 7$ & $<1.57 \mathrm{E} 4$ & 2.09E5 & $5.11 \mathrm{E} 6$ & $4.15 \mathrm{E} 6$ & $5.22 \mathrm{E} 7$ & $1.30 \mathrm{E} 5$ \\
\hline 442-A077 & $1.33 \mathrm{E} 7$ & $<1.50 \mathrm{E} 4$ & $2.07 \mathrm{E} 5$ & $4.41 \mathrm{E} 6$ & $3.90 \mathrm{E} 6$ & $5.49 \mathrm{E} 7$ & $1.14 \mathrm{E} 5$ \\
\hline 442-A078 & $1.28 \mathrm{E} 7$ & $<1.48 \mathrm{E} 4$ & $2.04 \mathrm{E} 5$ & $4.54 \mathrm{E} 6$ & $4.01 \mathrm{E} 6$ & $5.50 \mathrm{E} 7$ & $1.12 \mathrm{E} 5$ \\
\hline 442-A079 & $1.27 \mathrm{E} 7$ & $<1.48 \mathrm{E} 4$ & $2.00 \mathrm{E} 5$ & $4.56 \mathrm{E} 6$ & $3.95 \mathrm{E} 6$ & $5.32 \mathrm{E} 7$ & $1.20 \mathrm{E} 5$ \\
\hline 442-A081 & $1.23 \mathrm{E} 7$ & $<1.46 \mathrm{E} 4$ & $2.06 \mathrm{E} 5$ & $4.64 \mathrm{E} 6$ & 3.95E6 & $5.35 \mathrm{E} 7$ & $1.14 \mathrm{E} 5$ \\
\hline 442-A082 & $1.35 \mathrm{E} 7$ & $<1.48 \mathrm{E} 4$ & $2.14 \mathrm{E} 5$ & 4.67E6 & 4.17E6 & $5.76 \mathrm{E} 7$ & $1.15 \mathrm{E} 5$ \\
\hline 442-A083 & $1.32 \mathrm{E} 7$ & $<1.52 \mathrm{E} 4$ & $2.08 \mathrm{E} 5$ & $4.74 \mathrm{E} 6$ & $3.99 \mathrm{E} 6$ & $5.22 \mathrm{E} 7$ & $1.25 \mathrm{E} 5$ \\
\hline 442-A084 & $1.32 \mathrm{E} 7$ & $<1.49 \mathrm{E} 4$ & $2.15 \mathrm{E} 5$ & $4.55 \mathrm{E} 6$ & $3.97 \mathrm{E} 6$ & $5.40 \mathrm{E} 7$ & $1.15 \mathrm{E} 5$ \\
\hline 442-A085 & $1.20 \mathrm{E} 7$ & $<1.53 \mathrm{E} 4$ & $1.87 \mathrm{E} 5$ & $4.87 \mathrm{E} 6$ & $3.94 \mathrm{E} 6$ & $5.10 \mathrm{E} 7$ & $1.16 \mathrm{E} 5$ \\
\hline 442-A086 & $1.31 \mathrm{E} 7$ & $<1.50 \mathrm{E} 4$ & $1.83 \mathrm{E} 5$ & $4.40 \mathrm{E} 6$ & 3.97E6 & $5.48 \mathrm{E} 7$ & $1.19 \mathrm{E} 5$ \\
\hline Maximum & $1.68 \mathrm{E} 7$ & $4.65 \mathrm{E} 4$ & $2.75 \mathrm{E} 5$ & $6.83 \mathrm{E} 6$ & $5.55 \mathrm{E} 6$ & $6.44 \mathrm{E} 7$ & $1.58 \mathrm{E} 5$ \\
\hline Minimum & $9.57 \mathrm{E} 6$ & $1.39 \mathrm{E} 4$ & $1.55 \mathrm{E} 5$ & $3.53 \mathrm{E} 6$ & $3.08 \mathrm{E} 6$ & $4.05 \mathrm{E} 7$ & $9.76 \mathrm{E} 4$ \\
\hline Average $^{a}$ & $1.29 \mathrm{E} 7$ & $(2.3-16) \mathrm{E} 3$ & $2.02 \mathrm{E} 5$ & $4.78 \mathrm{E} 6$ & $4.06 \mathrm{E} 6$ & $5.29 \mathrm{E} 7$ & $1.19 \mathrm{E} 5$ \\
\hline$\% \mathrm{SD}^{a}$ & 9 & 359 & 9 & 11 & 9 & 8 & 10 \\
\hline
\end{tabular}

Note: Values are decay-corrected back to 1 day after the end of irradiation.

${ }^{a}$ Average and standard deviation don't include the five failed-TRISO particles (highlighted in gray), and the range for average ${ }^{110 \mathrm{~m}} \mathrm{Ag}$ retention spans the calculated average where the particles with undetectable ${ }^{110 \mathrm{~m}} \mathrm{Ag}$ inventory were summed using either the minimum possible value $(0 \mathrm{~Bq})$ or their individual quantification limits (denoted in table by a less than value in gray text). 
Table 5. Fraction of gamma-emitting isotopes retained in particles after safety test

\begin{tabular}{|c|c|c|c|c|c|c|c|c|}
\hline Tray:Number & Particle & ${ }^{106} \mathrm{Ru}$ & ${ }^{110 m} \mathrm{Ag}$ & ${ }^{125} \mathrm{Sb}$ & ${ }^{134} \mathrm{Cs}$ & ${ }^{137} \mathrm{Cs}$ & ${ }^{144} \mathrm{Ce}$ & ${ }^{154} \mathbf{E u}$ \\
\hline $1: 1$ & 442-A001 & 1.00 & $<0.36$ & 1.02 & 1.01 & 1.01 & 1.02 & 0.94 \\
\hline $1: 2$ & 442-A002 & 1.00 & $<0.33$ & 0.95 & 1.02 & 1.01 & 0.98 & 0.87 \\
\hline $1: 3$ & 442-A003 & 1.01 & $<0.33$ & 0.95 & 1.02 & 1.02 & 1.00 & 0.83 \\
\hline $1: 4$ & 442-A004 & 1.00 & $<0.34$ & 0.91 & 1.02 & 1.02 & 0.99 & 0.90 \\
\hline $1: 5$ & 442-A005 & 0.96 & $<0.81$ & 0.92 & 0.96 & 0.97 & 0.97 & 0.84 \\
\hline $1: 6$ & 442-A006 & 1.01 & $<0.18$ & 1.04 & 1.00 & 1.00 & 1.02 & 0.97 \\
\hline $1: 7$ & 442-A007 & 0.92 & $<0.51$ & 0.87 & 0.93 & 0.94 & 0.93 & 0.88 \\
\hline $1: 8$ & 442-A008 & 1.01 & $<0.36$ & 1.05 & 1.01 & 1.02 & 1.02 & 0.94 \\
\hline $1: 9$ & 442-A009 & 1.01 & $<0.28$ & 0.02 & 0.00 & 0.01 & 0.61 & 0.00 \\
\hline $1: 10$ & 442-A010 & 1.00 & $<0.36$ & 0.93 & 1.02 & 1.02 & 1.00 & 0.87 \\
\hline $1: 11$ & 442-A011 & 1.00 & $<0.39$ & 0.98 & 1.01 & 1.01 & 1.01 & 0.93 \\
\hline $1: 12$ & 442-A012 & 1.00 & $<0.42$ & 0.92 & 1.02 & 1.02 & 1.00 & 0.91 \\
\hline $1: 13$ & 442-A013 & 1.01 & $<0.30$ & 0.99 & 1.01 & 1.00 & 1.02 & 0.84 \\
\hline $1: 14$ & 442-A014 & 1.01 & $<0.34$ & 0.94 & 1.01 & 1.01 & 0.97 & 0.75 \\
\hline $1: 15$ & 442-A015 & 1.01 & $<0.43$ & 0.98 & 1.02 & 1.02 & 1.02 & 0.92 \\
\hline $2: 16$ & 442-A016 & 0.96 & $<0.44$ & 0.95 & 0.97 & 0.96 & 0.97 & 0.97 \\
\hline $2: 17$ & 442-A017 & 0.98 & $<0.40$ & 0.90 & 0.95 & 0.98 & 0.97 & 0.90 \\
\hline $2: 18$ & 442-A018 & 1.00 & $<0.36$ & 0.99 & 1.02 & 1.01 & 0.97 & 0.77 \\
\hline $2: 19$ & 442-A020 & 1.00 & $<0.36$ & 0.99 & 1.01 & 1.01 & 1.01 & 0.82 \\
\hline $2: 20$ & 442-A022 & 1.01 & $<0.34$ & 0.92 & 1.02 & 1.01 & 0.99 & 0.86 \\
\hline $2: 21$ & 442-A023 & 1.00 & $<0.41$ & 0.93 & 1.02 & 1.02 & 1.03 & 0.96 \\
\hline $2: 22$ & 442-A024 & 1.00 & $<0.51$ & 1.00 & 1.01 & 1.01 & 1.03 & 0.94 \\
\hline $2: 23$ & 442-A026 & 0.99 & $<0.33$ & 1.00 & 1.00 & 1.01 & 0.98 & 0.82 \\
\hline $2: 24$ & $442-\mathrm{A} 027$ & 1.00 & $<0.33$ & 0.87 & 1.00 & 1.01 & 1.03 & 0.95 \\
\hline $2: 25$ & 442-A028 & 1.00 & $<0.31$ & 0.96 & 1.01 & 1.00 & 1.01 & 0.93 \\
\hline $2: 26$ & 442-A029 & 0.99 & $<0.31$ & 0.94 & 1.01 & 1.01 & 1.01 & 0.86 \\
\hline $2: 27$ & 442-A030 & 0.99 & 0.63 & 1.00 & 1.00 & 1.01 & 1.01 & 0.95 \\
\hline $2: 28$ & 442-A031 & 1.00 & $<0.35$ & 1.01 & 1.01 & 1.01 & 1.01 & 0.92 \\
\hline $2: 29$ & 442-A032 & 1.00 & $<0.32$ & 0.96 & 1.00 & 1.02 & 1.01 & 0.92 \\
\hline $2: 30$ & 442-A033 & 1.00 & $<0.43$ & 0.99 & 1.00 & 1.01 & 1.00 & 0.94 \\
\hline $3: 31$ & 442-A034 & 0.99 & $<0.36$ & 0.95 & 1.00 & 1.01 & 1.02 & 0.94 \\
\hline $3: 32$ & 442-A037 & 1.00 & $<0.38$ & 0.94 & 1.01 & 1.01 & 1.01 & 0.87 \\
\hline $3: 33$ & 442-A038 & 1.01 & $<0.39$ & 0.96 & 0.94 & 1.01 & 1.02 & 0.87 \\
\hline $3: 34$ & 442-A040 & 1.00 & $<0.29$ & 0.94 & 1.00 & 1.00 & 0.98 & 0.83 \\
\hline $3: 35$ & 442-A041 & 1.00 & $<0.35$ & 0.93 & 1.00 & 1.01 & 0.99 & 0.87 \\
\hline $3: 36$ & 442-A042 & 0.97 & $<0.31$ & 0.90 & 0.99 & 0.97 & 0.97 & 0.86 \\
\hline $3: 37$ & 442-A043 & 1.00 & $<0.32$ & 0.96 & 1.00 & 1.01 & 0.98 & 0.78 \\
\hline $3: 38$ & 442-A044 & 1.00 & 0.49 & 0.97 & 1.00 & 1.02 & 1.02 & 0.98 \\
\hline $3: 39$ & 442-A045 & 0.99 & $<0.32$ & 0.92 & 1.01 & 1.01 & 0.99 & 0.90 \\
\hline $3: 40$ & 442-A046 & 0.99 & 0.85 & 1.03 & 1.00 & 1.01 & 1.02 & 0.99 \\
\hline $3: 41$ & 442-A047 & 0.99 & $<0.52$ & 0.97 & 1.01 & 1.01 & 1.02 & 0.98 \\
\hline
\end{tabular}


Table 5 (continued). Fraction of gamma-emitting isotopes retained in particles after safety test

\begin{tabular}{|c|c|c|c|c|c|c|c|c|}
\hline Tray:Number & Particle & ${ }^{106} \mathrm{Ru}$ & ${ }^{110 \mathrm{~m}} \mathrm{Ag}$ & ${ }^{125} \mathrm{Sb}$ & ${ }^{134} \mathrm{Cs}$ & ${ }^{137} \mathrm{Cs}$ & ${ }^{144} \mathrm{Ce}$ & ${ }^{154} \mathbf{E u}$ \\
\hline $3: 42$ & 442-A048 & 0.99 & $<0.33$ & 1.02 & 1.00 & 1.01 & 1.02 & 0.90 \\
\hline $3: 43$ & $442-\mathrm{A} 050$ & 0.99 & $<0.18$ & 0.76 & 0.01 & 0.01 & 1.02 & 0.04 \\
\hline $3: 44$ & 442-A051 & 0.99 & $<0.37$ & 0.99 & 1.00 & 1.00 & 0.97 & 0.76 \\
\hline $3: 45$ & 442-A052 & 0.99 & $<0.34$ & 1.00 & 1.01 & 1.02 & 1.03 & 0.91 \\
\hline $4: 46$ & 442-A053 & 0.99 & $<0.12$ & 0.03 & 0.00 & 0.01 & 0.08 & 0.01 \\
\hline $4: 47$ & 442-A055 & 0.99 & $<0.37$ & 0.98 & 1.00 & 1.01 & 0.98 & 0.72 \\
\hline $4: 48$ & 442-A056 & 1.00 & 0.31 & 0.94 & 1.01 & 1.00 & 1.00 & 0.89 \\
\hline $4: 49$ & 442-A057 & 1.00 & $<0.33$ & 0.96 & 0.93 & 1.01 & 0.99 & 0.82 \\
\hline $4: 50$ & 442-A058 & 0.99 & $<0.34$ & 0.93 & 1.00 & 1.00 & 1.01 & 0.98 \\
\hline $4: 51$ & 442-A059 & 1.00 & $<0.37$ & 0.98 & 1.01 & 1.01 & 0.98 & 0.72 \\
\hline $4: 52$ & 442-A060 & 0.99 & $<0.31$ & 1.00 & 0.99 & 1.00 & 1.00 & 0.92 \\
\hline $4: 53$ & 442-A061 & 1.00 & $<0.34$ & 0.92 & 0.99 & 1.01 & 0.97 & 0.88 \\
\hline $4: 54$ & 442-A062 & 0.98 & $<0.31$ & 0.93 & 1.01 & 1.00 & 0.98 & 0.80 \\
\hline $4: 55$ & 442-A063 & 0.98 & 0.50 & 0.97 & 0.98 & 0.99 & 0.99 & 1.01 \\
\hline $4: 56$ & 442-A064 & 0.99 & $<0.17$ & 0.02 & 0.00 & 0.01 & 0.01 & 0.00 \\
\hline $4: 57$ & 442-A065 & 1.00 & $<0.27$ & 0.93 & 1.01 & 1.01 & 0.98 & 0.79 \\
\hline $4: 58$ & 442-A067 & 0.99 & $<0.30$ & 0.98 & 1.00 & 1.00 & 1.00 & 0.83 \\
\hline $4: 59$ & 442-A068 & 0.99 & $<0.43$ & 0.95 & 1.00 & 1.00 & 1.00 & 0.92 \\
\hline $4: 60$ & 442-A070 & 1.00 & $<0.32$ & 0.93 & 1.00 & 1.01 & 1.00 & 0.88 \\
\hline $5: 61$ & 442-A071 & 0.99 & $<0.34$ & 0.85 & 1.01 & 1.00 & 0.98 & 0.90 \\
\hline $5: 62$ & 442-A072 & 1.00 & $<0.21$ & 0.02 & 0.01 & 0.01 & 0.15 & 0.01 \\
\hline $5: 63$ & 442-A073 & 0.99 & $<0.30$ & 0.98 & 1.00 & 1.00 & 1.00 & 0.79 \\
\hline $5: 64$ & 442-A074 & 1.00 & 1.09 & 1.04 & 1.00 & 1.01 & 1.00 & 0.99 \\
\hline $5: 65$ & 442-A075 & 1.00 & $<0.38$ & 1.00 & 1.01 & 1.01 & 1.02 & 0.95 \\
\hline $5: 66$ & 442-A076 & 1.00 & $<0.34$ & 1.02 & 1.02 & 1.02 & 1.02 & 0.95 \\
\hline $5: 67$ & 442-A077 & 1.00 & $<0.35$ & 1.01 & 1.01 & 1.02 & 1.03 & 0.92 \\
\hline $5: 68$ & 442-A078 & 1.00 & $<0.38$ & 0.98 & 1.02 & 1.02 & 1.01 & 0.87 \\
\hline $5: 69$ & 442-A079 & 1.01 & $<0.32$ & 0.99 & 1.01 & 1.02 & 1.04 & 0.93 \\
\hline $5: 70$ & 442-A081 & 1.00 & $<0.34$ & 1.04 & 1.02 & 1.02 & 1.03 & 0.90 \\
\hline $5: 71$ & 442-A082 & 1.00 & $<0.31$ & 0.95 & 1.02 & 1.02 & 1.02 & 0.88 \\
\hline $5: 72$ & 442-A083 & 1.00 & $<0.34$ & 1.01 & 1.01 & 1.02 & 1.03 & 0.94 \\
\hline $5: 73$ & 442-A084 & 1.00 & $<0.33$ & 1.05 & 1.01 & 1.01 & 1.02 & 0.90 \\
\hline $5: 74$ & 442-A085 & 1.00 & $<0.36$ & 0.97 & 1.02 & 1.01 & 1.04 & 0.88 \\
\hline $5: 75$ & 442-A086 & 1.00 & $<0.38$ & 0.88 & 1.01 & 1.01 & 1.01 & 0.93 \\
\hline \multicolumn{2}{|c|}{ Maximum } & $\begin{array}{c}1.01 \\
\end{array}$ & 1.09 & 1.05 & 1.02 & 1.02 & 1.04 & $\begin{array}{c}1.01 \\
\end{array}$ \\
\hline \multicolumn{2}{|c|}{ Minimum } & 0.92 & 0.31 & 0.85 & 0.93 & 0.94 & 0.93 & 0.72 \\
\hline \multicolumn{2}{|c|}{ Average $^{a}$} & 1.00 & $0.06-0.39$ & 0.96 & 1.00 & 1.01 & 1.00 & 0.89 \\
\hline \multicolumn{2}{|c|}{$\% \mathrm{SD}^{a}$} & 1.4 & 356 & 5 & 2 & 1.4 & 2 & 7 \\
\hline
\end{tabular}

Note: Values were determined from the ratio of activity after the test over activity before the test (Table 3 and Table 4).

${ }^{a}$ Average and standard deviation don't include the five failed-TRISO particles (highlighted in gray), and the range for average ${ }^{110 \mathrm{~m}} \mathrm{Ag}$ retention spans the calculated average where the particles with undetectable ${ }^{110 \mathrm{~m}} \mathrm{Ag}$ inventory were summed using either the minimum possible value (0) or their individual quantification limits (denoted in table by a less than value in gray text). 
In addition to six-hour counting of all the particles, the five graphite particle trays and graphite nut, which served as a lid for the top tray (Figure 1), were also individually gamma counted (Table 6). After gamma counting, these components were analyzed together by burn-leach (data shown in the last row of Table 6). Results of burn-leach compared well with gamma scanning except for the ${ }^{144} \mathrm{Ce}$, which was more susceptible to error in the estimated gamma counting efficiency.

Table 6. Activity in Bq/particle of gamma-emitting isotopes in graphite trays (Figure 1)

\begin{tabular}{|c|c|c|c|c|c|c|c|}
\hline Tray & ${ }^{106} \mathrm{Ru}$ & ${ }^{110 \mathrm{~m}} \mathrm{Ag}$ & ${ }^{125} \mathrm{Sb}$ & ${ }^{134} \mathrm{Cs}$ & ${ }^{137} \mathrm{Cs}$ & ${ }^{144} \mathrm{Ce}$ & ${ }^{154} \mathrm{Eu}$ \\
\hline \multirow{2}{*}{ Nut } & $<1.03 \mathrm{E} 4$ & $<1.82 \mathrm{E} 4$ & $<8.28 \mathrm{E} 2$ & $<4.70 \mathrm{E} 2$ & $1.12 \mathrm{E} 3$ & 7.43E6 & $2.90 \mathrm{E} 3$ \\
\hline & $(<0.0008)$ & $(<0.39)$ & $(<0.003)$ & $(<0.00010)$ & $(0.0003)$ & $(0.14)$ & $(0.018)$ \\
\hline \multirow{2}{*}{5} & $5.21 \mathrm{E} 4$ & $<4.05 \mathrm{E} 4$ & $<1.87 \mathrm{E} 3$ & $<1.08 \mathrm{E} 3$ & $1.11 \mathrm{E} 3$ & $5.15 \mathrm{E} 7$ & $<1.32 \mathrm{E} 3$ \\
\hline & $(0.004)$ & $(<0.86)$ & $(<0.006)$ & $(<0.0002)$ & $(0.0003)$ & $(0.99)$ & $(<0.008)$ \\
\hline \multirow{2}{*}{4} & $6.69 \mathrm{E} 4$ & $<5.54 \mathrm{E} 4$ & $<2.51 \mathrm{E} 3$ & $<1.45 \mathrm{E} 3$ & $5.51 \mathrm{E} 2$ & $8.96 \mathrm{E} 7$ & $<1.78 \mathrm{E} 3$ \\
\hline & $(0.005)$ & $(<1.17)$ & $(<0.008)$ & $(<0.0003)$ & $(0.00013)$ & $(1.72)$ & $(<0.011)$ \\
\hline \multirow{2}{*}{3} & $<1.75 \mathrm{E} 4$ & $<3.12 \mathrm{E} 4$ & $<1.43 \mathrm{E} 3$ & $<8.33 \mathrm{E} 2$ & $5.73 \mathrm{E} 2$ & $2.85 \mathrm{E} 7$ & $<1.10 \mathrm{E} 3$ \\
\hline & $(<0.0013)$ & $(<0.66)$ & $(<0.005)$ & $(<0.00017)$ & $(0.00014)$ & $(0.55)$ & $(<0.007)$ \\
\hline \multirow{2}{*}{2} & $<1.20 \mathrm{E} 4$ & $<2.16 \mathrm{E} 4$ & $<9.76 \mathrm{E} 2$ & $<5.55 \mathrm{E} 2$ & $1.17 \mathrm{E} 3$ & $1.32 \mathrm{E} 7$ & 3.32E3 \\
\hline & $(<0.0009)$ & $(<0.46)$ & $(<0.003)$ & $(<0.00011)$ & $(0.0003)$ & $(0.25)$ & $(0.02)$ \\
\hline \multirow{2}{*}{1} & $<1.87 \mathrm{E} 4$ & $<3.35 \mathrm{E} 4$ & $<1.53 \mathrm{E} 3$ & $<8.79 \mathrm{E} 2$ & $3.15 \mathrm{E} 2$ & $3.27 \mathrm{E} 7$ & $<1.07 \mathrm{E} 3$ \\
\hline & $(<0.0014)$ & $(<0.71)$ & $(<0.005)$ & $(<0.00018)$ & $(0.00008)$ & $(0.63)$ & $(<0.007)$ \\
\hline \multirow{2}{*}{$\operatorname{Sum}^{\mathrm{a}}$} & $1.19-1.77 \mathrm{E} 5$ & $0.00-2.00 \mathrm{E} 5$ & $0.00-9.14 \mathrm{E} 3$ & $0.00-5.27 \mathrm{E} 3$ & $4.84 \mathrm{E} 3$ & $2.23 \mathrm{E} 8$ & $0.73-1.15 \mathrm{E} 4$ \\
\hline & $(0.009-0.14)$ & $(0.00-4.25)$ & $(0.00-0.03)$ & $(0.00-0.011)$ & $(0.0012)$ & $(4.28)$ & $(0.04-0.07)$ \\
\hline \multirow{2}{*}{$\begin{array}{l}\text { Sum from } \\
\text { burn-leach }\end{array}$} & $<1.11 \mathrm{E} 5$ & $<7.49 \mathrm{E} 4$ & $<5.09 \mathrm{E} 3$ & $1.68 \mathrm{E} 3$ & $1.06 \mathrm{E} 4$ & $2.69 \mathrm{E} 8$ & $8.48 \mathrm{E} 3$ \\
\hline & $(<0.008)$ & $(<1.6)$ & $(<0.016)$ & $(0.0003)$ & $(0.003)$ & $(5.16)$ & $(0.05)$ \\
\hline
\end{tabular}

Note: Values are decay-corrected back to 1 day after the end of irradiation.

${ }^{a}$ The sum is reported as a range where undetectable inventories were summed using either the minimum possible value $(0)$ or their individual quantification limits (denoted in table by a less than value in gray text).

Particles were loaded in numerical order (Table 5) from the bottom to the top of the stack (e.g., Tray 1 contained Particles 442-A001-A015). Trays 1, 3, and 5 each held one particle with failed TRISO, Tray 4 held two, and Tray 2 held none. However, since the trays and top nut also acted as lids for the tray below, they could also be exposed to particles with failed TRISO in the tray below. In increasing magnitude of direct exposure, Tray 2 and the nut were exposed to one failed TRISO from below, Trays 1 and 3 each held one failed TRISO, Tray 5 held one failed TRISO and was exposed to two more from below, and Tray 4 had the highest exposure with two failed TRISO inside the tray and one below. A detectable but still low inventory of ${ }^{106} \mathrm{Ru}$ was measured in Trays 4 and 5 , which were exposed to the highest number of failed-TRISO particles; this suggests a very small amount of ruthenium was released to the trays through failed-TRISO, but not enough to measure with IMGA as a reduced inventory in the particles. Significant silver, antimony, and cesium were released by the failed-TRISO particles (Table 5) but these isotopes 
were not significantly retained in the surrounding graphite. A very small amount of ${ }^{137} \mathrm{Cs}$ was detected on the trays, but the amount was negligible compared to what was released and may include a measurable contribution from hot cell contamination. Similarly, a small amount of ${ }^{154} \mathrm{Eu}$ was detected that was again negligible compared to the near $100 \%$ release from each failed-TRISO particle. In contrast, an appreciable amount of the cerium released from the particles with failed TRISO was retained in the tray stack. In fact, 4.28 particles' inventory of ${ }^{144} \mathrm{Ce}$ was measured in the tray stack by IMGA and 5.16 by burn-leach, compared to 3.13 particles' inventory released by the particles. The additional ${ }^{144} \mathrm{Ce}$ may be due to cerium release from particles with intact $\mathrm{SiC}$. The high retention of cerium in the graphite nut and trays is consistent with the lower detected level in the graphite bolt and holder ( 0.27 particles' inventory) and no detectable ${ }^{144} \mathrm{Ce}$ in the furnace outside the graphite. Trays 4 and 5 , which were exposed to the most failed TRISO, held the most ${ }^{144} \mathrm{Ce}$.

\section{MICROSTRUCTURAL ANALYSIS}

\subsection{Particles with Failed TRISO - X-ray Tomography and Optical Microscopy}

After IMGA measurements were complete, the five particles that exhibited high radioisotope release (highlighted in Table 4 and Table 5) were mounted in special holders and examined using 3D x-ray tomography. After x-ray imaging, these particles were mounted for materialography and polished sections were prepared using the 3D x-ray data to guide the grinding and polishing process. The procedures used for materialographic and $\mathrm{x}$-ray sample preparation and imaging are described in a previous report [Hunn et al. 2013a]. Figure 11 through Figure 16 show the defect structures as imaged by x-ray tomography and optical microscopy. Grayscale intensity in the tomography images increases with increasing x-ray absorption, thus regions darker than surrounding material indicate lower density volumes (e.g., void spaces in the layers or carbon-rich zones in the $\mathrm{SiC}$ ), while regions brighter that the surrounding material indicate deposits of higher density material (e.g., clusters of uranium and metallic fission products in the layers). These high density deposits also tend to appear as higher intensity regions in the optical micrographs due to higher reflectivity. Apparent kernel structure in the x-ray images is an artifact of the tomographic reconstruction due to the high absorption of $\mathrm{x}$-rays in the kernel.

Full-TRISO failure has not been observed in any other AGR-1 safety test conducted in the CCCTF, and occurred in only one $1800^{\circ} \mathrm{C}$ test in the FACS [Demkowicz et al. 2015a, 323-325]. Multiple-particle SiC failure without TRISO failure was observed in AGR-1 Compacts 5-1-3 and 3-2-3 after heating to $1800^{\circ} \mathrm{C}$ [Hunn et al. 2014e], and this failure involved localized degradation of the $\mathrm{SiC}$ at the tip of an IPyC fracture that produced a carbon-filled pathway through otherwise undamaged $\mathrm{SiC}$. In these failed-SiC particles, the OPyC layer presented the last line of defense against krypton release. The fact that krypton release was relatively prevalent in the $1800^{\circ} \mathrm{C}$ furnace test of loose particles from Compact 4-4-2, while it was rarely observed in particles that experienced $\mathrm{SiC}$ failure during $1800^{\circ} \mathrm{C}$ safety testing of AGR-1 compacts, is likely due to a reduction in OPyC layer performance as a result of the removal of the surrounding matrix and the possible impact of deconsolidation and particle handling on the OPyC layer integrity. Structural support from the surrounding matrix may be a contributing factor to the resistance of the $\mathrm{OPyC}$ to fracture in particles whose $\mathrm{SiC}$ failed during compact safety testing.

With the exception of Particle 442-A050 (Figure 16), four of the five TRISO-failed particles exhibited significant dispersion of kernel material into the surrounding layers, and some of the kernels were noticeably reduced in volume. This unusual lack of stability and material retention by the kernel was presumably related to the TRISO failure. Because the TRISO failure allowed various elements and compounds to easily migrate out of the particle during the safety test, certain reactions and diffusion processes may have been able to proceed unchecked that would otherwise be limited in a closed system with intact coating layers. For instance, it is well-known that the reaction where carbon reduces uranium oxide can proceed at safety test temperatures of $1600-1800^{\circ} \mathrm{C}$ if a sufficient partial pressure of carbon monoxide is not maintained by the gas-tight coating layers [Wilhelm 1964]. 
All five particles with failed TRISO had cracks through the SiC layer that extended over a significant arc (e.g., Figure 12c, and Figure 13c, Figure 16c), as opposed to the localized degradation holes typically observed in AGR-1 particles with failed SiC. Chemical degradation of the $\mathrm{SiC}$ surrounding the crack was observed in several of the materialographic cross sections (e.g., Figure $12 \mathrm{f}$ and Figure 14f) and was also evident in the x-ray images. It cannot be determined from the post-safety test microstructural analysis whether $\mathrm{SiC}$ fracture occurred before or after the onset of $\mathrm{SiC}$ degradation. However, based on particle performance during other AGR-1 safety tests, it is reasonable to assume that the observed SiC fracture was not solely caused by the exposure to $1800^{\circ} \mathrm{C}$, given that it has not been observed in any of the wholecompact safety tests. SiC failure may have started with the typical AGR-1 degradation mechanism [Hunn et al. 2014b] followed by eventual fracture of the weakened layer, based on the observation that each particle had IPyC cracks that exposed the inner surface of the $\mathrm{SiC}$ layer at the degradation site (e.g., Figure 12f, Figure 15a, and Figure 16a).
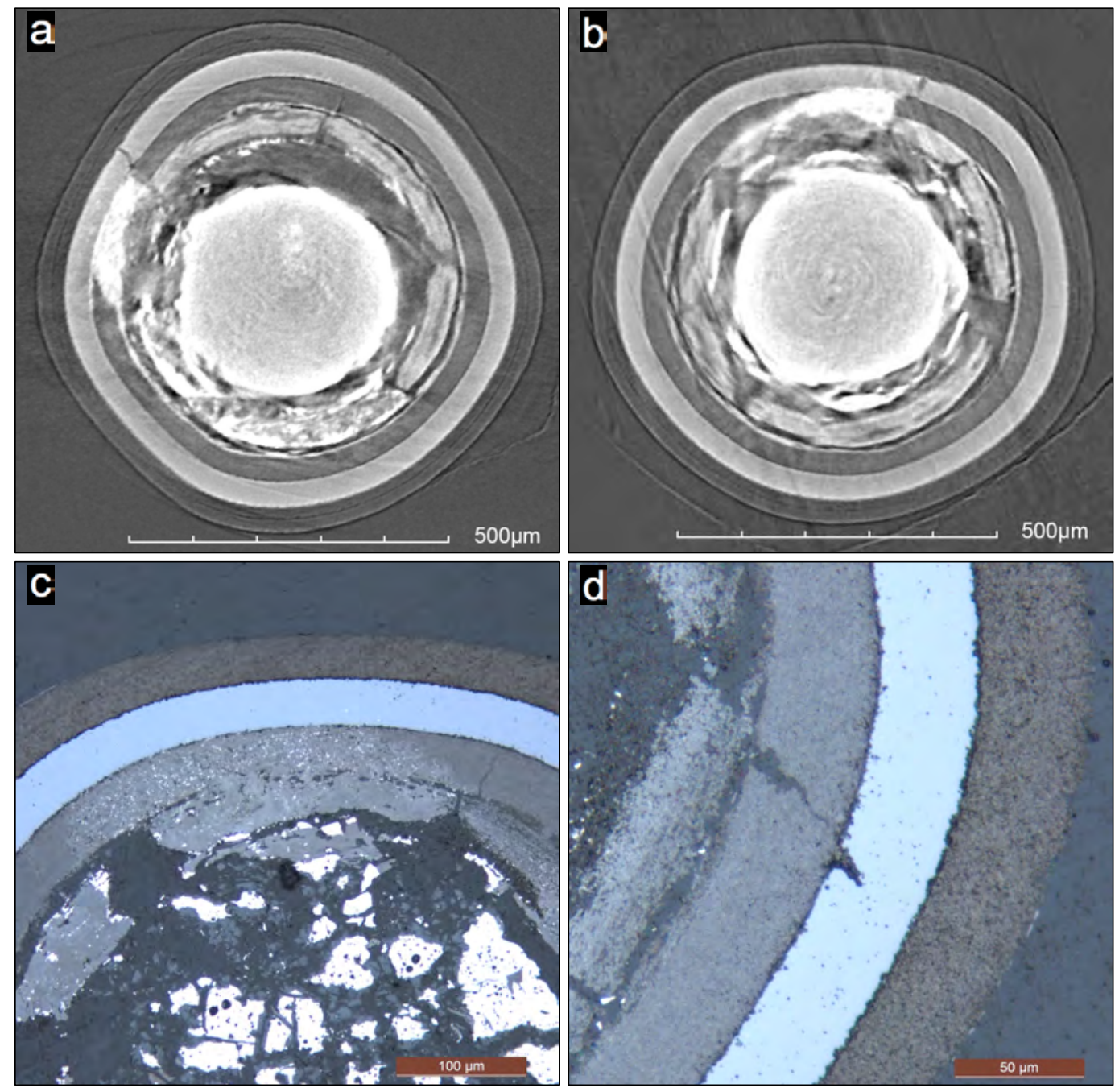

Figure 11. Particle 442-A009: (a and b) oblique orthogonal $x$-ray tomographs showing SiC crack aligned with buffer/IPyC crack, with adjacent U-dispersion in IPyC; (c) optical micrograph of a polished section showing $\mathrm{U}$ dispersion in the IPyC; (d) another area in the same polished section where typical local SiC degradation at the tip of an IPyC crack was observed that was not associated with the TRISO failure. 

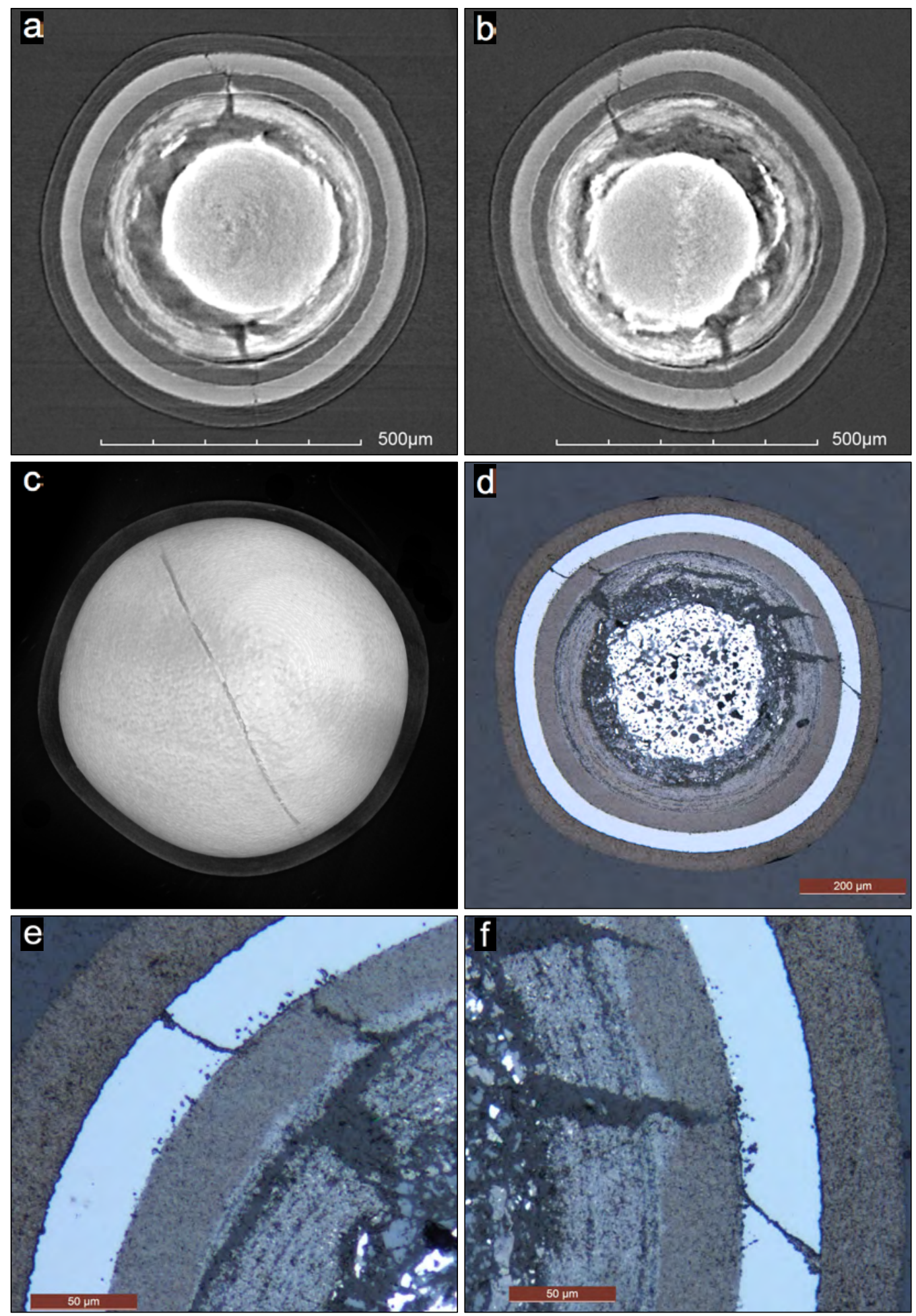

Figure 12. Particle 442-A064: (a and b) oblique orthogonal x-ray tomographs showing SiC crack at top and bottom of image and noticeable loss of kernel material; (c) 3D visualization using threshold filter to display $\mathrm{SiC}$ surface (OPyC layer is semitransparent) to show extended $\mathrm{SiC}$ crack that almost circumnavigated this particle, $(\mathrm{d}-\mathrm{f})$ optical micrographs of polished section showing $\mathrm{SiC}$ crack and degradation at IPyC/SiC interface. 

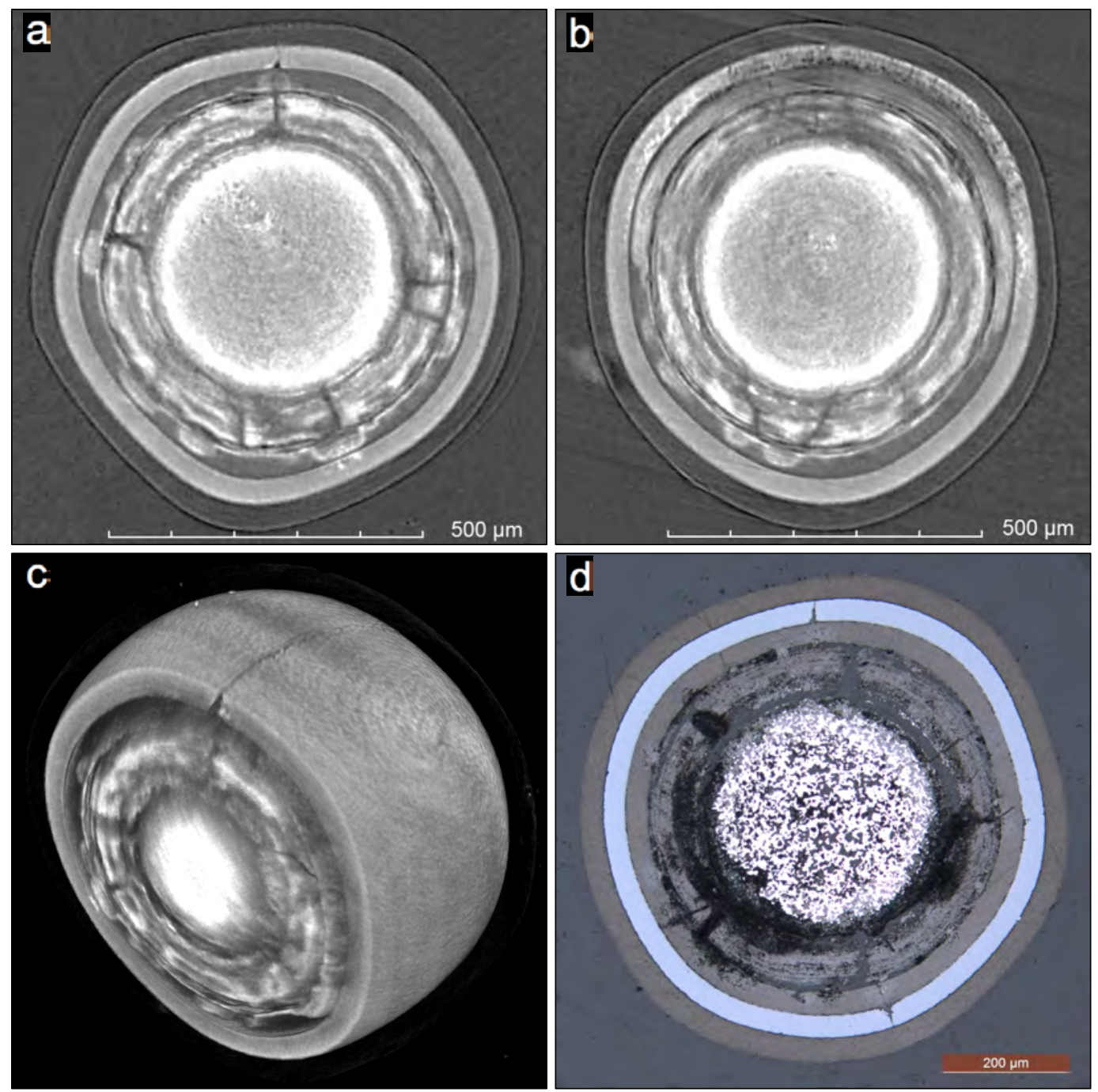

$50 \mu \mathrm{m}$

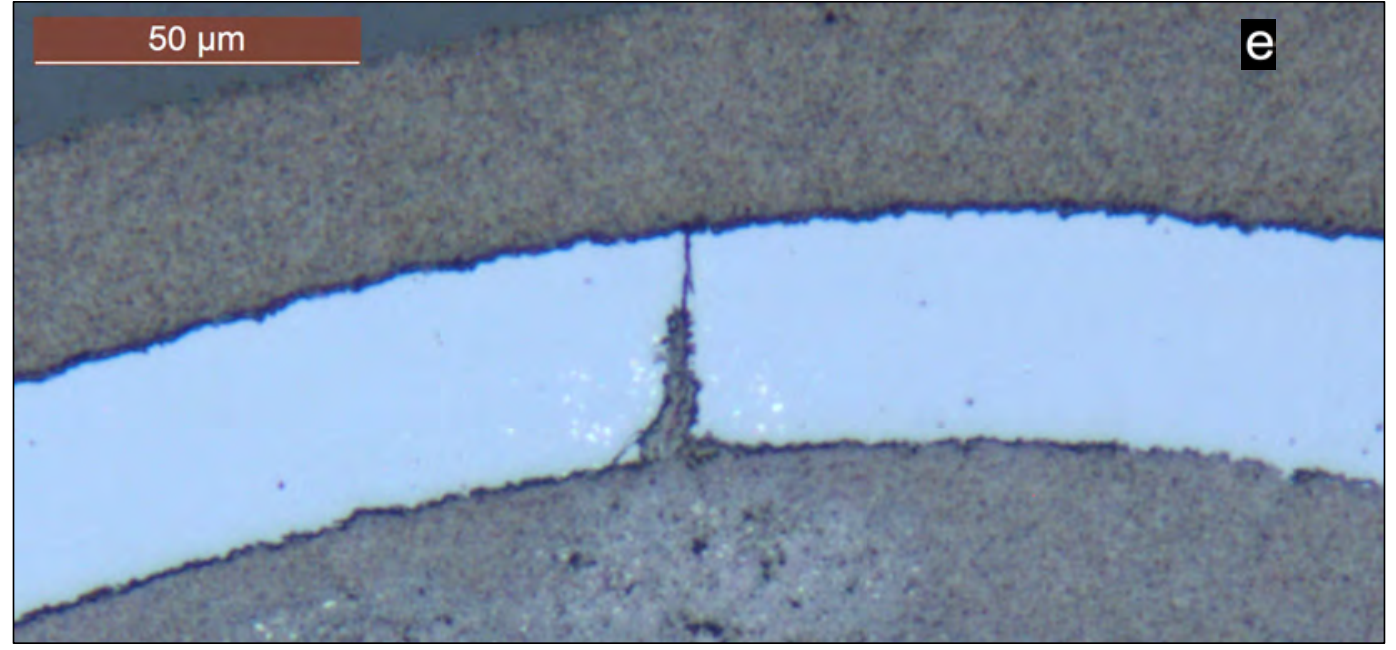

Figure 13. Particle 442-A053: (a and b) oblique orthogonal $x$-ray tomographs showing through-layer SiC degradation aligned along an IPyC crack and significant uranium dispersion throughout the buffer and IPyC, (c) sectioned 3D visualization using a threshold filter to emphasize $\mathrm{SiC}$ and higher-density material (pyrocarbon not visible) and showing an extended $\mathrm{SiC}$ crack with degradation, (d and e) optical micrographs of polished section showing metallic clusters in $\mathrm{SiC}$ and degradation extending out into $\mathrm{SiC}$ along the crack. 

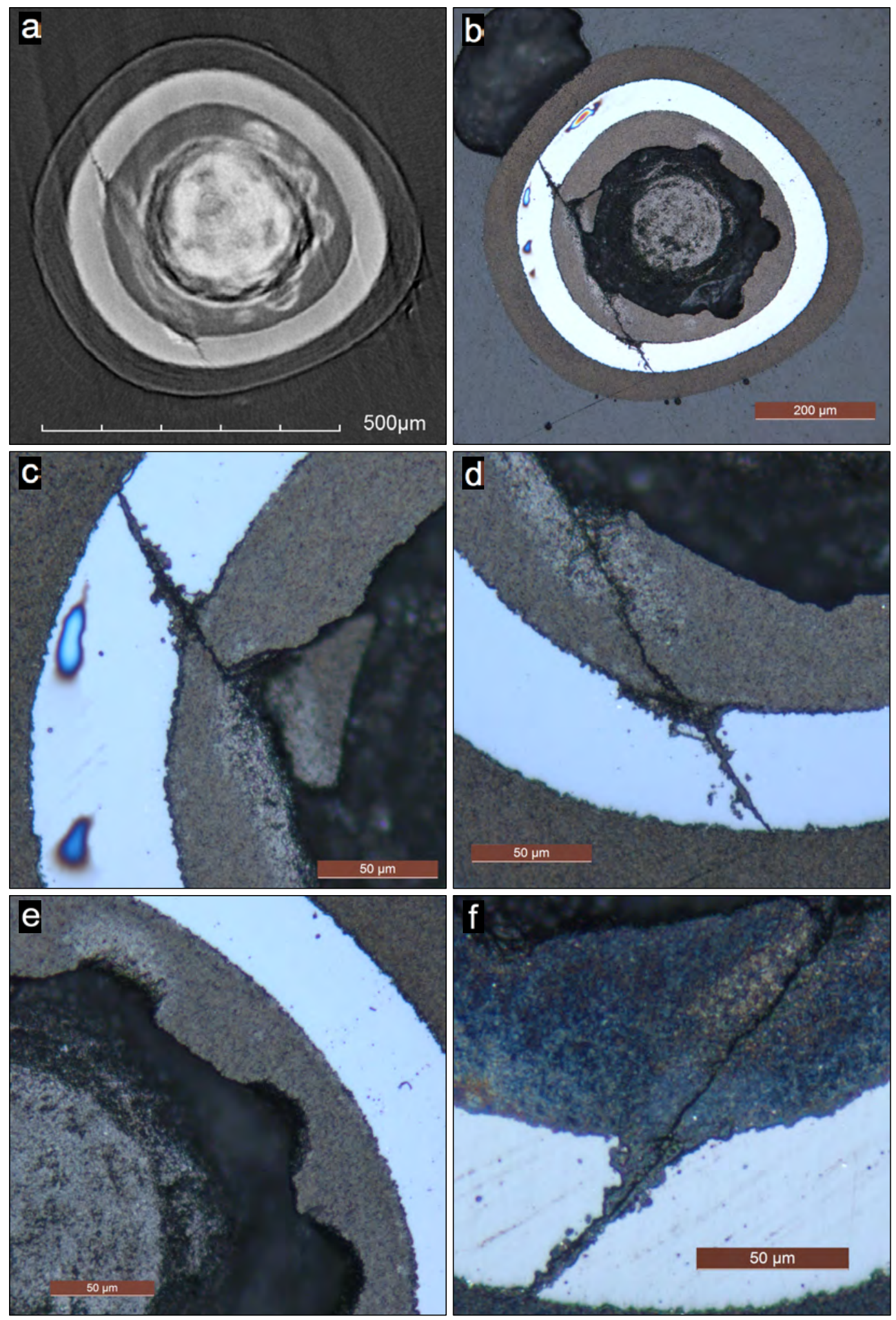

Figure 14. Particle 442-A053: Set of images obtained further from midplane compared to Figure 13; (a) x-ray tomograph showing same plane as (b), (b-e) optical micrograph of polished section showing SiC degradation along crack and missing pockets of IPyC associated with radial dispersions of uranium visible in (a), (f) SiC degradation in another plane that was parallel and close to (c) showing a crack through the degraded region. 

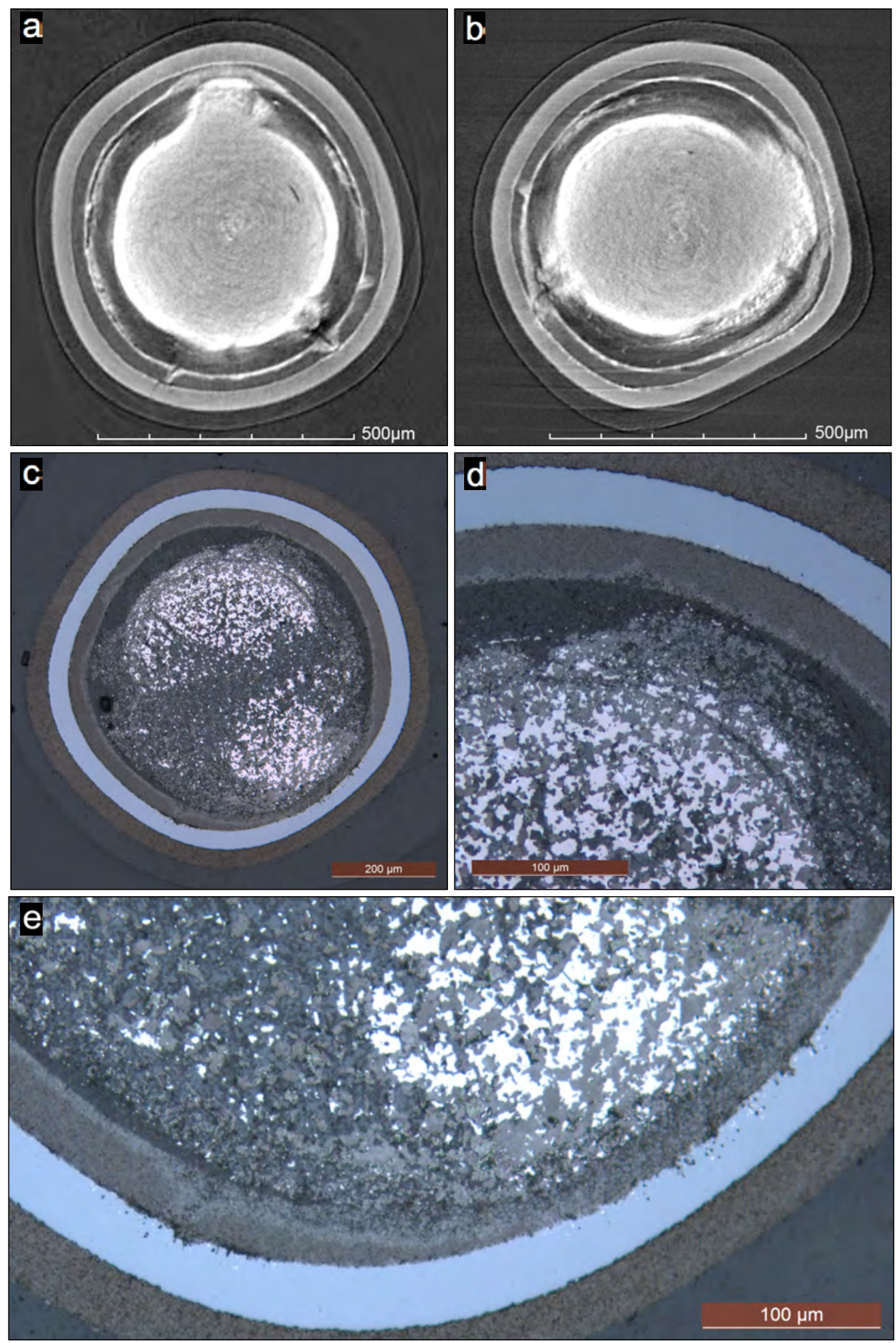

Figure 15. Particle 442-A072: (a and b) oblique orthogonal x-ray tomographs showing SiC crack (lower left) and kernel protrusion with significant local dispersion in IPyC with related SiC degradation that did not penetrate the layer, $(\mathrm{c}-\mathrm{e})$ optical micrographs of polished section showing kernel reaction with buffer and degradation in the IPyC and SiC where kernel material had protruded to the IPyC. 

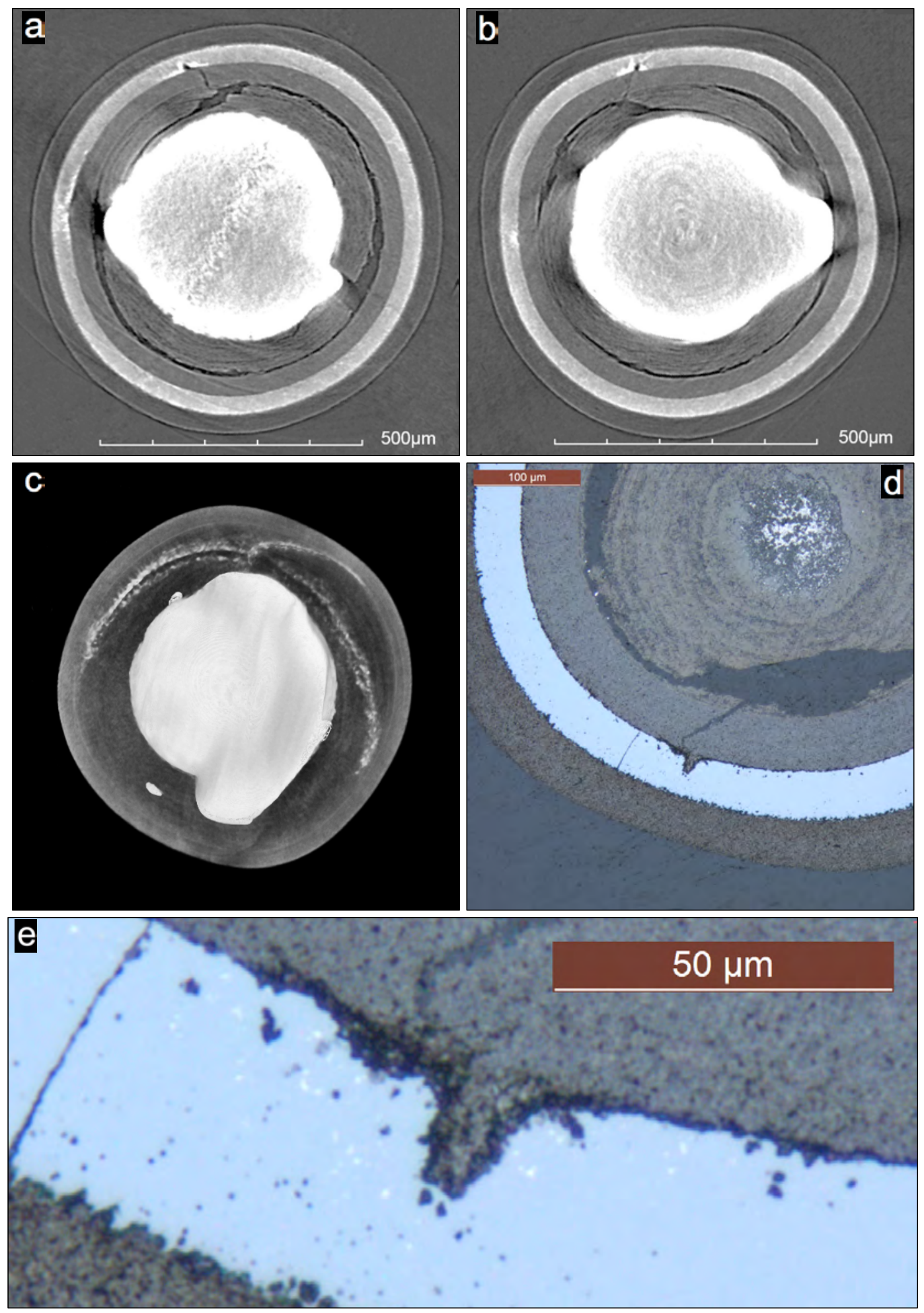

Figure 16. Particle 442-A050: (a and b) oblique orthogonal $x$-ray tomographs showing $\mathrm{SiC}$ degradation through layer at tip of IPyC crack associated with edge of buffer delamination, (c) 3D semitransparent visualization showing kernel protrusion and extended $\mathrm{SiC}$ degradation along a crack in the $\mathrm{SiC}$, (d and e) optical micrographs of polished section showing SiC degradation at tip of IPyC crack. 
Figure 11a and Figure 11b show a pair of orthogonal planes passing through the approximate center of Particle 442-A009. These images provide two views of a crack in the $\mathrm{SiC}$ that was closely aligned with cracks in the buffer and IPyC layers. The SiC crack extended above and below the image plane in Figure 11a but was not as long as the cracks observed in some of the other particles with failed TRISO. The $\mathrm{x}$-ray imaging was not able to resolve any through-layer pathways or cracks in the $\mathrm{OPyC}$, and none were apparent in the polished section; however, based on the release of krypton and other fission products from this particle, an OPyC failure is suspected. A dense, triangular-shaped cloud of higher-density elements was observed in the IPyC on one side of the crack (Figure 11a and Figure 11b). The optical micrograph in Figure 11c shows a cross section through this unusual feature, which was identified by EDS as containing predominantly uranium deposits (see Section 5.2). Significant uranium dispersion from the kernel was also evident throughout the entire buffer layer, with heavy pileup at the outside surface of the buffer, where it was detached from the IPyC. Figure 11d shows a region away from the TRISO failure containing an IPyC crack with localized SiC degradation more typical of what has been observed in failed-SiC particles from $1800^{\circ} \mathrm{C}$ safety-tested AGR-1 compacts; however, this region did not appear to be associated with the TRISO failure, because the $\mathrm{SiC}$ degradation did not penetrate the layer and the IPyC crack was isolated from the failed region discussed above. Particle 442-A009 released most of its silver, antimony, cesium and europium, but retained more than half of its cerium (Table 5).

Figure 12a and Figure 12b show orthogonal x-ray tomographs through the center of Particle 442-A064; cracking of the $\mathrm{SiC}$ is evident at the the top and bottom of both images. These features are part of a single crack extending almost all the way around the particle. Figure $12 \mathrm{c}$ is a $3 \mathrm{D}$ visualization using a threshold filter to display the crack on the $\mathrm{SiC}$ surface (the OPyC layer is presented as semitransparent). Similar to Particle 442-A009, the kernel in Particle 442-A064 exhibited a noticeable volume reduction and there was a considerable amount of kernel material dispersed in the buffer. This particle, however, did not show the same large-area dispersion in the IPyC seen in Figure 11b and Figure 11c. The optical micrographs in Figure 12 show a cross section where a small offset is evident between the IPyC crack and the SiC crack and degradation can be seen along the IPyC/SiC interface in the crack locations. Examination of the tomography data in three dimensions showed that this offset varied along the length of the crack and the $\mathrm{SiC}$ crack was aligned with the IPyC crack in some locations, which suggests that the SiC crack may have initiated where it was aligned with the IPyC crack and then deviated from direct alignment as it propagated around the particle. The polished cross section also revealed some uranium dispersion in the IPyC, radiating in from the buffer near the IPyC crack. As with Particle 442-A009, obvious cracks in the OPyC (expected based on the krypton release observed in the CCCTF) were not observed; however, $\mathrm{x}$-ray tomography indicated the possible presence of localized low-density regions or very small cracks in the $\mathrm{OPyC}$ at the limit of the $\mathrm{x}$-ray resolution. There was no visible OPyC crack circumnavigating the particle adjacent to the $\mathrm{SiC}$ crack, as would be expected if these two layer fractured as a unit, and OPyC degradation was not evident in the polished cross section. Particle 442-A064 released greater than 98\% of all the isotopes reported in Table 5, except for ruthenium (which was retained in all five failed-TRISO particles).

Figure 13a and Figure 13b are orthogonal tomographs through the midplane of Particle 442-A053, and Figure $13 \mathrm{c}$ is a $3 \mathrm{D}$ representation that shows a crack in the SiC layer . A cross section of the crack is shown at the top of Figure 13a such that it is perpendicularly intersecting the tomograph plane. In Figure $13 \mathrm{~b}$, the image plane is aligned along the crack direction, such that the structure in the SiC near the crack can be seen along most of the crack length; severe degradation and fission product deposits can be seen along the entire length of the crack in the top half of this SiC cross section in Figure 13b. Similar to the two particles discussed above, Particle 442-A053 also experienced significant dispersion of kernel material into the buffer and IPyC layers. Dispersion into the IPyC was observed to be localized and occurred at numerous positions around the sphere. X-ray tomography revealed numerous semispherical zones of heavy elements that appeared to radiate from single point sources around the buffer/IPyC interface; the dispersed material in these nodules was predominantly uranium, as determined by elemental analysis in the SEM discussed below (see Section 5.2). Figure 13a and Figure 13b show several of the 
zones; and at two locations in Figure 13a, degradation of the $\mathrm{SiC}$ is evident where the the zones extended all the way through the IPyC.

Figure 14 shows a series of micrographs of a planar section through Particle 442-A053 that was revealed part way through the process of grinding down to the plane shown in Figure 13d. The materialographic specimen was lightly polished at this intermediate plane to examine the nodule-like uranium-rich regions in the IPyC that were visible in the matching x-ray tomograph (Figure 14a). (The bright region in the center of Figure 14a is not the kernel; it is a planar section through the buffer with high $\mathrm{x}$-ray absorption due to high uranium content.) The material in the nodules was not retained in the polished section (Figure $14 \mathrm{~b}$ and Figure 14e), except for one location at the edge of the uppermost nodule in Figure 14e. The others left a rounded void where material had fallen out during grinding and polishing. The friable nature of these regions indicate significant reaction between the intruding uranium and the carbon microstructure of the IPyC.

Figure 13d and Figure 13e show significant SiC degradation occurred at the site where the SiC was fractured. Figure 13e shows a brown area inside the degraded region that looks optically similar to the carbon material in the IPyC in this brightfield, reflected-light image. Surrounding this central carbon-rich region are numerous bright spots in the $\mathrm{SiC}$ due to uranium deposits in the SiC. Similar structure was evident in the other polished plane revealed in Figure 14c and Figure 14d, and Figure $14 \mathrm{f}$ shows the same area as Figure 14c prior to polishing to reveal the plane in Figure 14c. Figure 14f and Figure 13e both clearly show a crack through the center of the carbon-rich degraded zone in the SiC. Whether the crack preceded or followed the $\mathrm{SiC}$ degradation is a matter for debate. The fact that the degradation is fairly symmetric about the crack suggests that the crack occurred first. Imaging and elemental analysis in the SEM of the polished plane shown in Figure 13 is discussed in Section 5.2. Particle 442-A053 exhibited fission product release behavior somewhere between Particle 442-A009 and Particle-A064, releasing most of its silver, antimony, cesium and europium, but retaining $\sim 8 \%$ of its cerium (Table 5).

Release behavior in Particle 442-A072 was similar to Particle 442-A053, with slightly more cerium retention $(\sim 15 \%)$ in Particle 442-A072. Figure 15a and Figure 15b are orthogonal x-ray tomographs through the center of the particle. Particle 442-A072 exhibited uranium dispersion into the buffer that was less widespread than that in Particle 442-A053, and tended to be localized in regions where the buffer appeared to be fractured. The large protrusion of high- $Z$ material visible in Figure $15 \mathrm{a}$ and Figure $15 \mathrm{~b}$ is similar to features observed in many as-irradiated AGR-1 particles, where kernel material was observed to protrude into a gap formed as the buffer fractured [Hunn, Savage, and Kehn 2012; Ploger et al. 2012]. However, the extent of interaction of this protruding kernel material with the surrounding buffer resulted in a microstructure that was different from the typical kernel protrusion. Materialographic specimen preparation and examination was focused on a region where this kernel protrusion was in contact with the $\mathrm{IPyC}$, rather than on the $\mathrm{SiC}$ failure that was located elsewhere in the particle. Figure $15 \mathrm{c}$ is an optical micrograph of the entire cross section and shows that some kernel/buffer reaction occurred all the way around the interface. Figure $15 \mathrm{~d}$ shows a region where the kernel/buffer interaction extended close to the buffer/IPyC interface and there is evidence of associated IPyC degradation at this location. Figure 15e shows an area where dense kernel material was in intimate contact with the inside surface of the IPyC; at its thinnest point, about half of the IPyC thickness appears to have been consumed by reaction with the kernel. In addition, uranium dispersion into the IPyC can be seen radiating from the edge of the denser kernel material. Uranium dispersion can also be seen radiating out from the crack in the IPyC visible in the lower left corner of Figure 15e. In the observed cross section, $\mathrm{SiC}$ degradation was most advanced in the $\mathrm{SiC}$ adjacent to the thinned section of pyrocarbon (right side of Figure 15e) and at the IPyC crack (lower left corner of Figure 15e). The SiC layer also appeared to be thinner in the middle of the degraded region closest to the kernel protrusion, again suggesting that material at the inside surface was reacting more thoroughly with the kernel material. Additional SEM and EDS analysis of this region is discussed in Section 5.2. Despite the wide affected area and extent of the SiC degradation near the kernel protrusion, the observed fission product release in the CCCTF does not appear to be related to this degradation. X-ray tomography revealed a crack in the $\mathrm{SiC}$ on the opposite side of the particle (Figure 15a). The SiC crack 
was aligned with a crack in the IPyC that had typical features observed in other AGR-1 failed SiC particles, namely, the IPyC cracking was related to buffer fracture and fission product concentration in the $\mathrm{IPyC}$ crack and at the exposed $\mathrm{SiC}$ interface was observed in conjunction with $\mathrm{SiC}$ degradation.

Figure 16a and Figure 16b show orthogonal x-ray tomographs through the center of Particle 442-A050. A crack in the IPyC was clearly visible in the $\mathrm{x}$-ray tomographs and this crack led to localized SiC degradation, where a wide, low-density region in the $\mathrm{SiC}$ was surrounded by relatively-high pileup of heavy elements (predominantly uranium, based on EDS analysis). The IPyC crack and SiC degradation extended along a line that traversed more than halfway around the particle. Figure $16 \mathrm{c}$ shows a $3 \mathrm{~d}-$ visualization that emphasizes the high and low density regions in the $\mathrm{SiC}$ along this crack. The $\mathrm{x}$-ray images also show that significant kernel material had protruded into the gap created by the fractured buffer, and the kernel was in contact with the IPyC in one area; however, this contact did not lead to the same interaction discussed above for Particle 442-A072 and the kernel protrusion had a microstructure more like what has been observed in as-irradiated AGR-1 Compact 4-4-2 particles [Hunn et al. 2013a]. Figure 16d and Figure 16e show a polished planar section that revealed part of the IPyC crack and degraded $\mathrm{SiC}$ (the crack in the $\mathrm{SiC}$ to the left of the degraded region in these micrographs is probably an artifact of the grinding process). The degraded $\mathrm{SiC}$ region was filled with a carbon-rich material similar to that observed in Particle 442-A053 (Figure 13e), which was evidently left behind when silicon was removed from the silicon carbide originally occupying this space. Surrounding the carbon-rich zone, numerous bright spots from uranium deposits in the $\mathrm{SiC}$ are visible, as well as dark spots indicating pockets of silicon depletion.

Despite the extensive and heavily-degraded crack in the SiC shown in Figure 16c, fission product release from Particle 442-A050 was less advanced than in the other failed-TRISO particles (Table 5). Cesium retention was almost zero, as in all the failed-TRISO particles, but cerium retention was essentially $100 \%$. Antimony was also mostly retained, in contrast to the other failed-TRISO particles. Congruent with the higher cerium and antimony retention, dispersion of kernel material into the surrounding carbon layers was also absent. The reduced uranium dispersion and higher retention of cerium and ruthenium in this particle may be related to the timing of the $\mathrm{SiC}$ and TRISO failures. Cesium and krypton release detected in the CCCTF (Figure 2) indicated that one of the five failed-TRISO particles failed much later in the test.

\subsection{Particles with Failed TRISO-SEM with EDS}

Scanning electron microscopy analysis of the low-cesium particles focused on the anomalous regions and compromised TRISO layers identified by optical microscopy and x-ray tomography analysis, the SEM analysis served to supplement the observations from these techniques. Energy-dispersive spectroscopy was performed on select areas to identify the composition of unique features of interest. Imaging was conducted using both secondary-electron imaging (SEI) and backscattered-electron composition (BEC); the SEI mode provides topographical information, while the BEC signal is dependent on the local atomic number $(Z)$ of the incident substrate. The $Z$-contrast enhancement from BEC was used to identify high- $Z$ fission products within the TRISO layers. The SEM and EDS analysis was performed on a JEOL 6390LV tungsten-filament SEM equipped with an Oxford X-Max50 silicon-drift detector for EDS.

As discussed in Section 5.1, the polished cross section of Particle 442-A009 revealed a region of degraded $\mathrm{SiC}$ at the tip of an IPyC crack (Figure 11d) that was typical of what has been observed in failed-SiC particles from $1800^{\circ} \mathrm{C}$ safety-tested AGR-1 compacts. However, this region did not appear to be associated with the TRISO failure because the $\mathrm{SiC}$ degradation did not penetrate the layer and was isolated from the extended $\mathrm{SiC}$ crack that was obvious in the x-ray images (Figure 11a and b). Figure 17 shows a BEC micrograph of the region around the degraded $\mathrm{SiC}$ shown in Figure 11d. In Figure 17, the high-concentration of bright spots in the buffer are clusters of high-Z elements from the kernel (predominantly uranium); this dispersed kernel material was responsible for the elevated x-ray absorption seen throughout the buffer of Particle 442-A009 (Figure 11a and b). Figure 17 also shows a lower concentration of high- $\mathrm{Z}$ elements in the $\mathrm{SiC}$, near the IPyC/SiC interface, and in the IPyC, except in the 
IPyC region adjacent to the IPyC fracture. This depletion of high- $Z$ elements in the IPyC adjacent to an IPyC crack has been observed in failed-SiC particles from other AGR-1 safety-tests [Hunn et al. 2014b] and is thought to be the result of localized interaction with the $\mathrm{SiC}$ drawing this material to the reaction site.

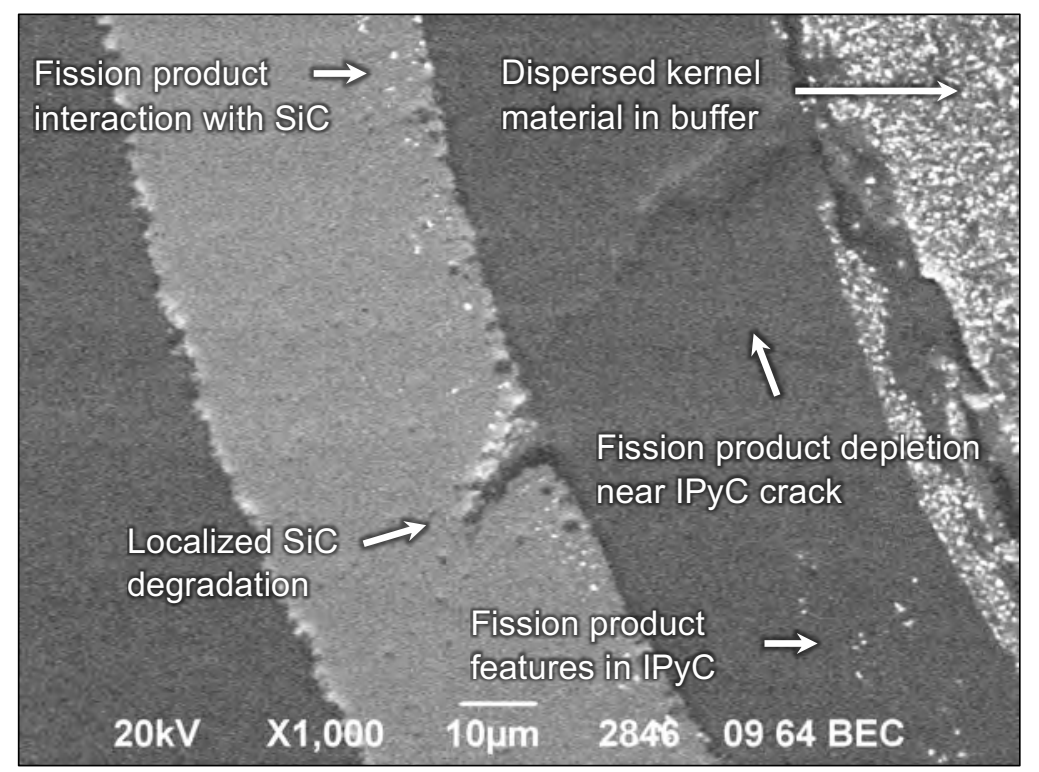

Figure 17. BEC micrograph from Particle 442-A009 showing the localized SiC degradation at the tip of an IPyC crack also shown in Figure 11d.

Figure 18 shows the region of IPyC discussed in Section 5.1 that exhibited a high-concentration of high-Z elements only on one side of the crack through the buffer, IPyC, and SiC (Figure 11a-c). It is evident that there was also a high frequency of fission product features in the SiC layer adjacent to this IPyC region. This behavior differs from the nature of the fission product distribution observed in the SiC layer away from the high-Z accumulation area in the IPyC. Notably, a lower frequency of features were observed in the $\mathrm{SiC}$ layer and at depths limited to the inside half of the SiC layer (Figure 19 and Figure 17). Spot analysis with EDS revealed that the material in the IPyC layer was predominantly uranium, although some molybdenum was also detected at the IPyC/SiC interface. In the $\mathrm{SiC}$, the bright spots coincided with high concentrations of uranium and, in a few cases, traces of ruthenium. Silicon was also observed in the IPyC, presumably as a result of the migration of silicides formed in the SiC.
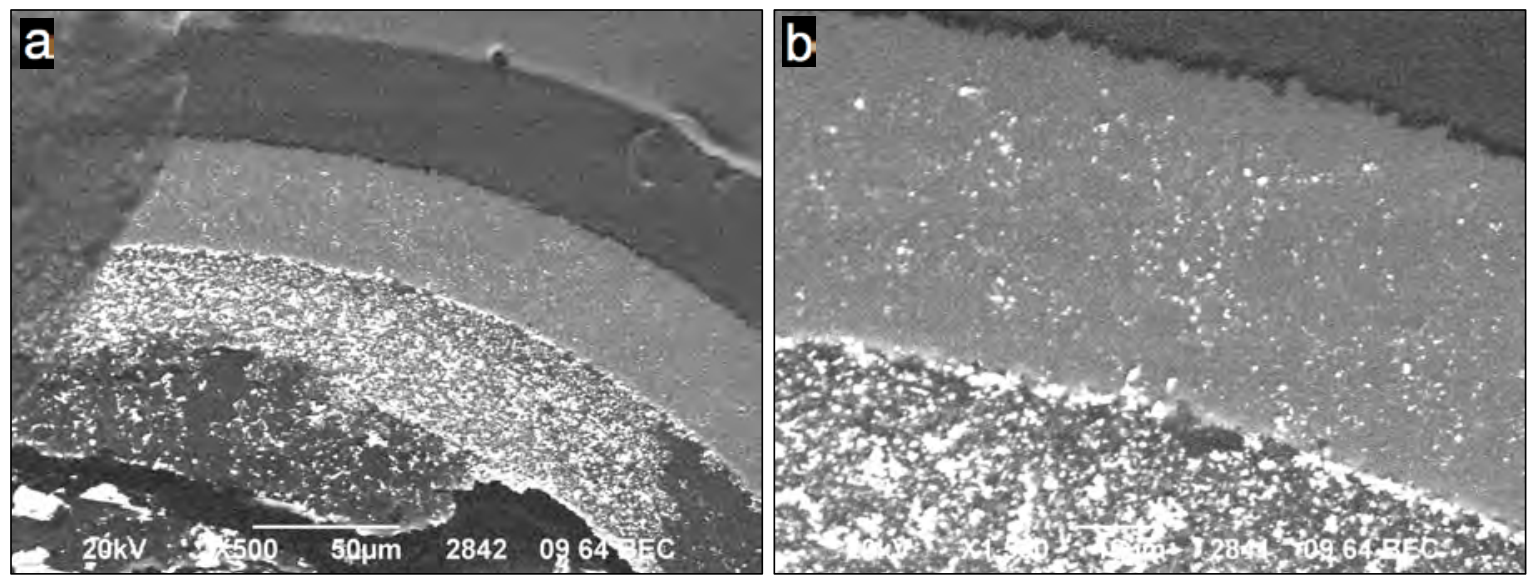

Figure 18. BEC micrographs of Particle 442-A009 showing two magnifications of the region near the through-layer crack with a large, concentrated dispersion of high-Z features in the IPyC and $\mathrm{SiC}$. 

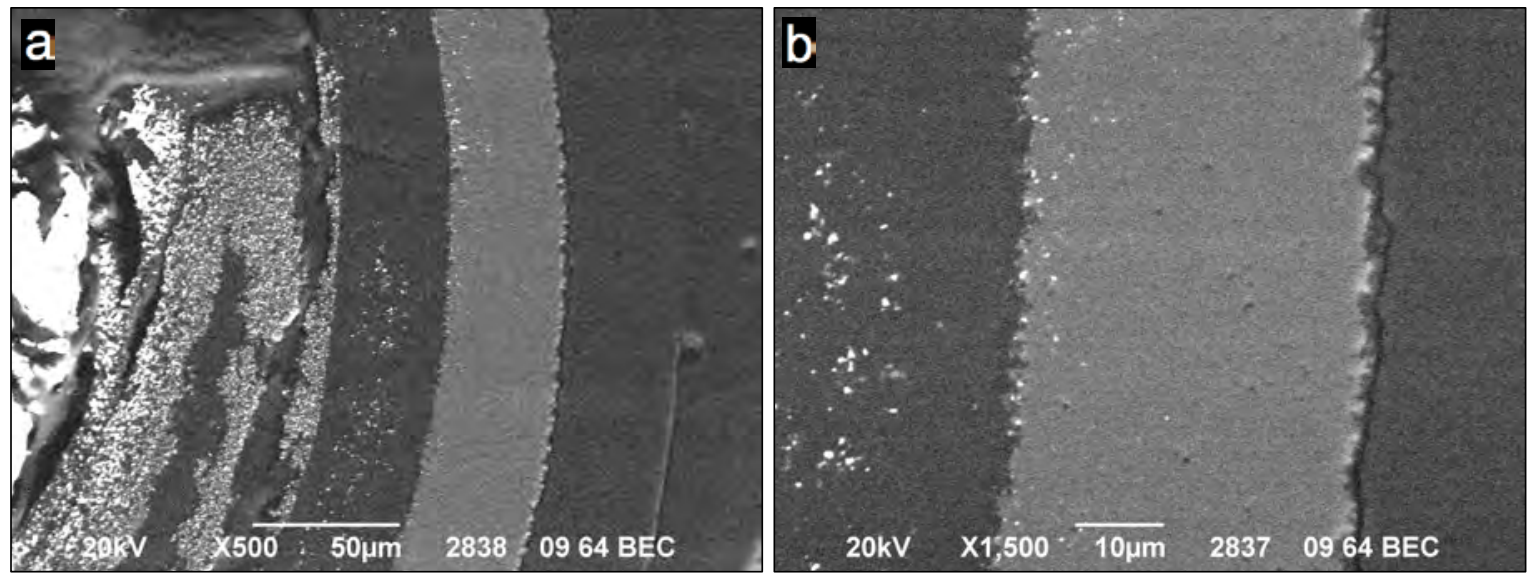

Figure 19. BEC micrographs of Particle 442-A009 for comparison to Figure 18 showing two magnifications of a region away from the through-layer crack with more typical dispersion of high-Z features.

Figure 20 shows two SiC regions in Particle 442-A064 away from the SiC crack, one adjacent to an IPyC crack and one bordered by intact IPyC. The bright features were identified by EDS analysis as primarily due to clusters of uranium. The concentrations of silicon and carbon in the clusters could not be explicitly determined due to beam interaction with the surrounding SiC. As has been typically observed in AGR-1 PIE [Demkowicz 2015b], the distribution of these uranium-rich features in the SiC layer was more limited where IPyC cracks were not observed.
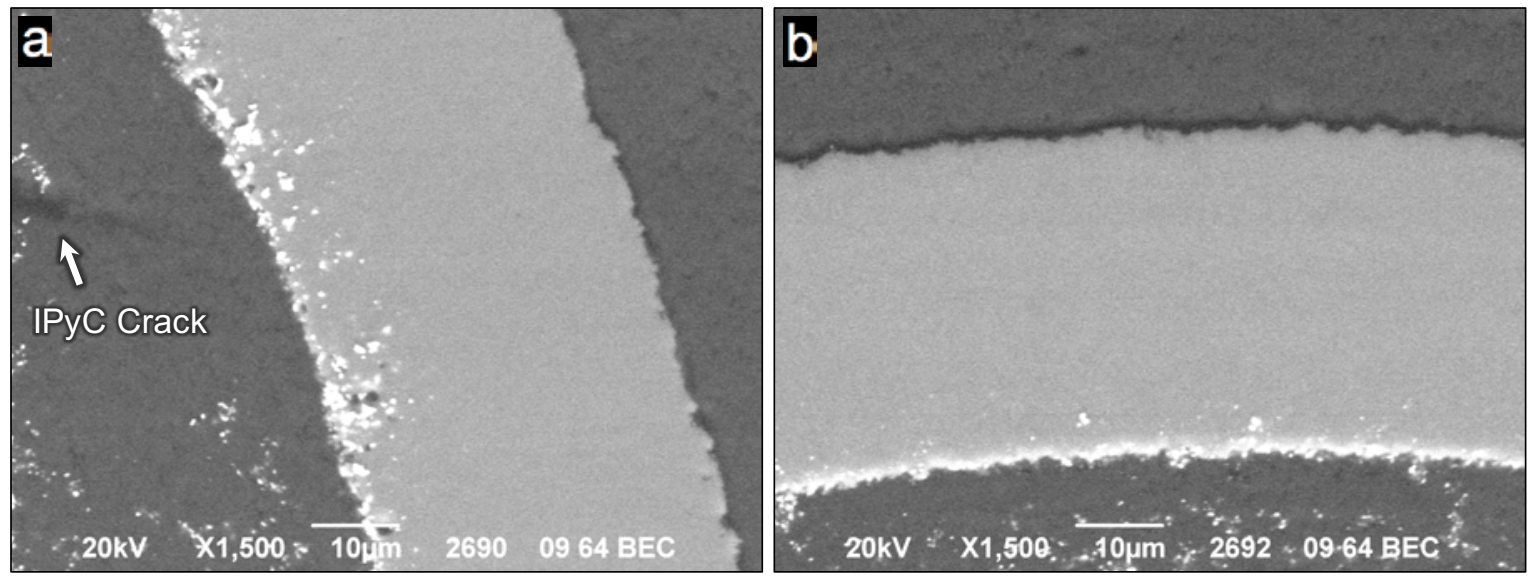

Figure 20. BEC micrographs of Particle 442-A064 showing the distribution of uranium features in the SiC layer in (a) a region with an IPyC crack near the IPyC/SiC interface, and (b) an area away from any cracks in the TRISO layers.

Figure 21 shows an SEI micrograph of the same region of Particle 442-A064 shown in Figure 12f. A lateral crack in the IPyC, close to the IPyC SiC interface, can be seen connecting the buffer/IPyC crack to the $\mathrm{SiC}$ crack. As discussed in Section 5.1, this offset probably indicates that the SiC crack did not initiate at this location, and the connecting crack may have occurred as the $\mathrm{SiC}$ crack propagated around the particle. Figure 21 also shows x-ray intensity maps for uranium, silicon, and carbon. The uranium map shows deposits of uranium along the IPyC/SiC interface at the tip of the IPyC crack on the side opposite the $\mathrm{SiC}$ crack, but along the IPyC/SiC interface between the IPyC crack and the $\mathrm{SiC}$ crack there was a notable reduction in the clusters of uranium or other fission product species. This suggests that the cracks may have served as a pathway for release of these species from the particle. The bright regions in the buffer and IPyC in the SEI micrograph in Figure 21 and the x-ray images in Figure 12 were identified by the EDS mapping to be predominantly uranium and carbon, and no significant concentrations of other species were detected. Mapping of the carbon content revealed a carbon-rich zone along the $\mathrm{SiC}$ crack; 
this is especially evident in Figure 21 where the crack widens at the outer edge of the $\mathrm{SiC}$ and suggests local decomposition of the $\mathrm{SiC}$ at the crack surface.
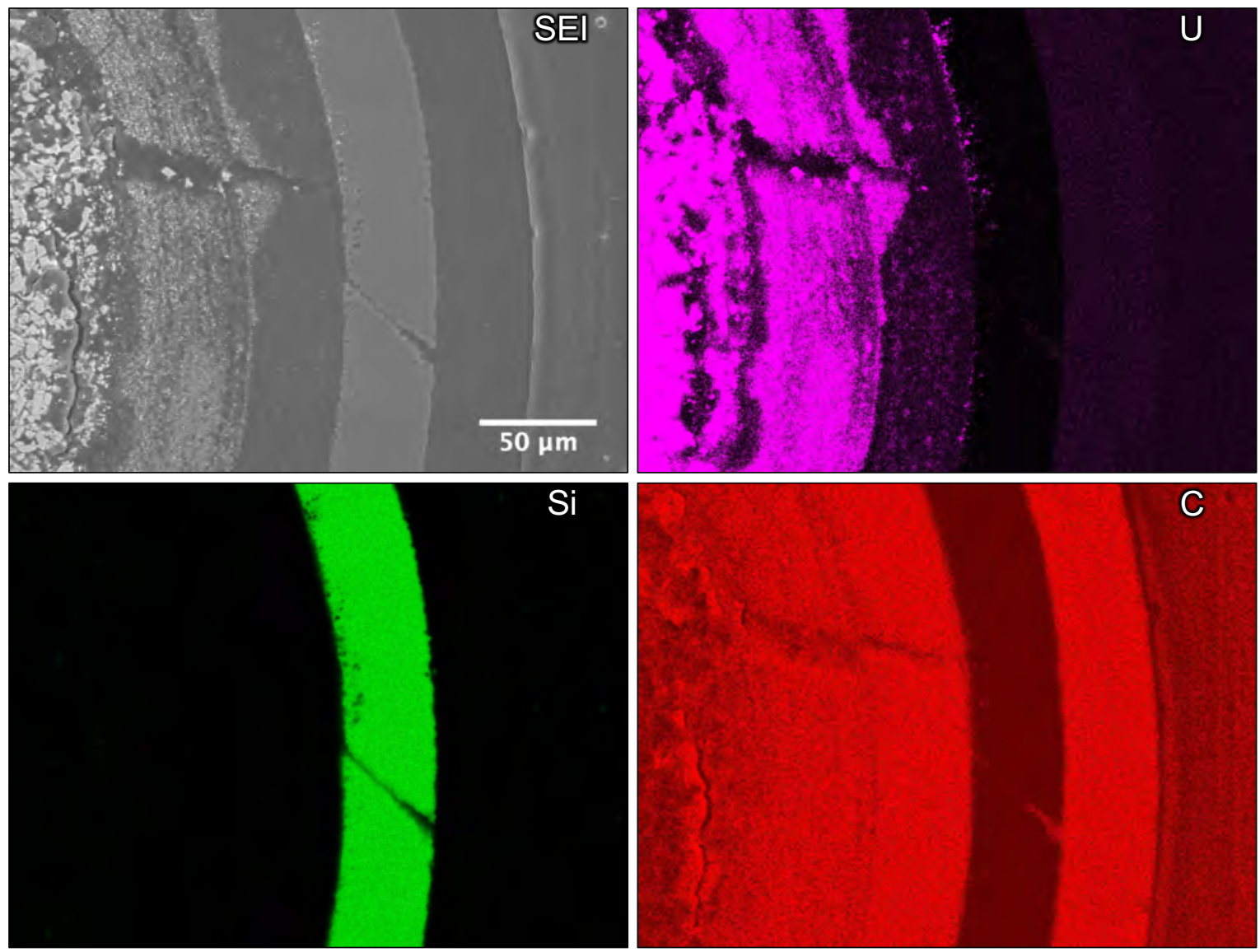

Figure 21. SiC fracture area in Particle 442-A064 showing SEI micrograph and corresponding EDS maps of $x$-ray intensity indicating concentration profiles of uranium, silicon, and carbon.

The x-ray tomography and optical microscopy analysis of Particle 442-A053 showed several unique features including a through-layer crack in the $\mathrm{SiC}$, bright lobes of high- $\mathrm{Z}$ material in the IPyC layer, and prevalent dispersion of uranium in the buffer (Figure 13). These unique features were further analyzed by SEM. Figure 22 shows the distribution of uranium in the buffer. Spot analysis indicated the dispersed uranium features did not contain significant concentrations of other species. Figure 23 shows two areas away from the crack in the $\mathrm{SiC}$ layer. Away from the crack, the distribution of uranium-rich features in the $\mathrm{SiC}$ varied depending on the presence of uranium in the IPyC layer. In Figure 23a, numerous high- $\mathrm{Z}$ features can be seen in the $\mathrm{SiC}$ layer adjacent to one of the lobe-shaped regions of uranium dispersion in the IPyC layer. Figure 23a also shows carbon-rich zones in the SiC layer that indicate $\mathrm{SiC}$ decomposition at the $\mathrm{IPyC} / \mathrm{SiC}$ interface where the lobe-shaped uranium dispersion in the IPyC was in close proximity to the $\mathrm{SiC}$. Figure $23 \mathrm{~b}$ is representative of regions in the $\mathrm{SiC}$ layer not situated near a high concentration of uranium in the IPyC, where high- $\mathrm{Z}$ features embedded in the $\mathrm{SiC}$ layer were not as prominent and were observed to be smaller. These areas also showed a typical interlaced $\mathrm{IPyC} / \mathrm{SiC}$ interface that was essentially unchanged from that generated during the $\mathrm{SiC}$ layer deposition, except for a few clusters of high- $Z$ elements in the interface region. EDS analysis revealed that all of the high- $Z$ features observed in the $\mathrm{SiC}$ layer were uranium-rich, with no significant intensity from other elements. 

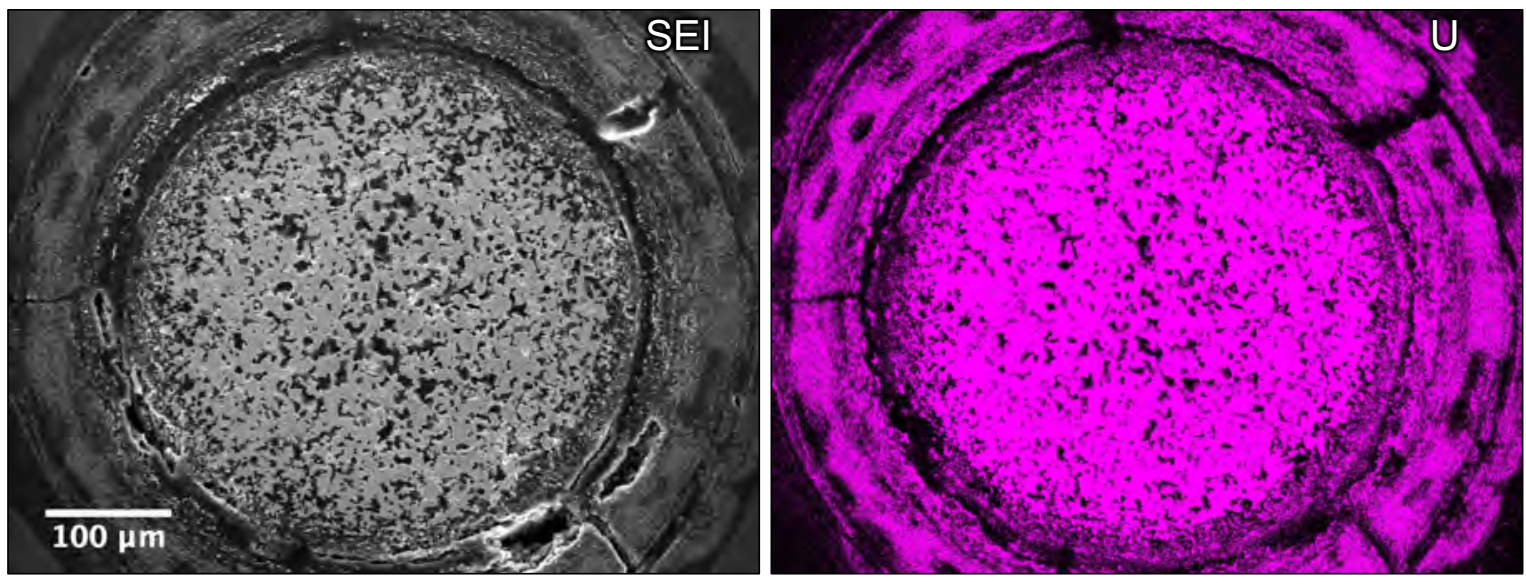

Figure 22. SEI micrograph and corresponding EDS x-ray intensity map of the uranium concentration showing the dispersion of uranium in the buffer of Particle 442-A053.
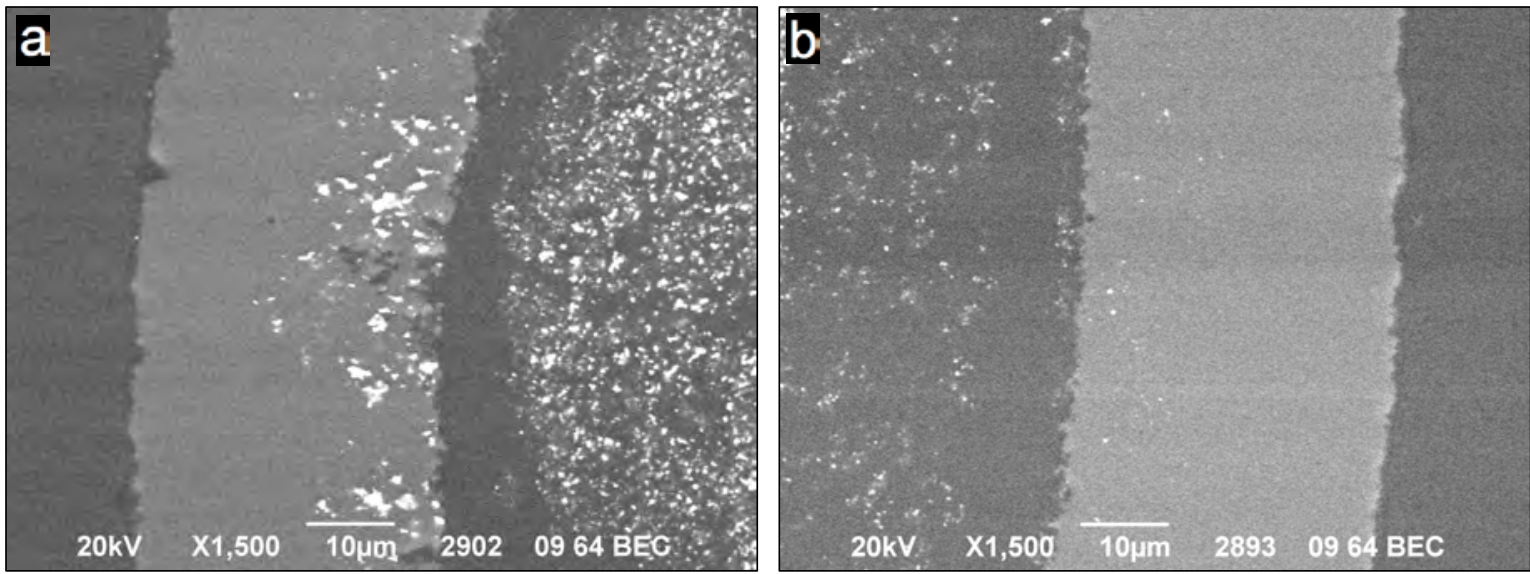

Figure 23. Particle 442-A053 SEM micrographs showing (a) the SiC layer next to a uranium dispersion lobe with significant $\mathrm{SiC}$ attack and (b) the $\mathrm{SiC}$ layer with no adjacent uranium dispersion lobe in the IPyC layer.

The images presented in Figure 24 and Figure 25 show the SEM analysis of a region surrounding a through-layer crack in the $\mathrm{SiC}$ of Particle 442-A053 (also shown in Figure 13). Figure 24a shows the degraded region on either side of the crack was filled with a dense material that EDS determined to be mostly carbon (Figure 25), and the $\mathrm{SiC}$ around this degraded region was populated with high- $\mathrm{Z}$ features determined by EDS to be predominantly uranium and depleted of silicon (Figure 25). No other significant intensity from other elements were observed, with the exception of zirconium found in a single analyzed feature. The EDS mapping in Figure 25 also confirmed the crack was positioned next to a uranium-rich lobe in the IPyC layer. While it is difficult to draw firm conclusions as to the timing of the formation of the through-layer crack in the $\mathrm{SiC}$, Figure 25 does suggest some relationship to the enhanced degradation of the $\mathrm{SiC}$ in the vicinity of the lobe-shaped uranium dispersion in the IPyC layer. These lobe-like features were observed to be associated with $\mathrm{SiC}$ degradation in regions away from the $\mathrm{SiC}$ crack (e.g, Figure 23), suggesting they may have formed prior to the SiC crack; and it is reasonable to assume that $\mathrm{SiC}$ failure could be facilitated by local decomposition of the $\mathrm{SiC}$ layer. 

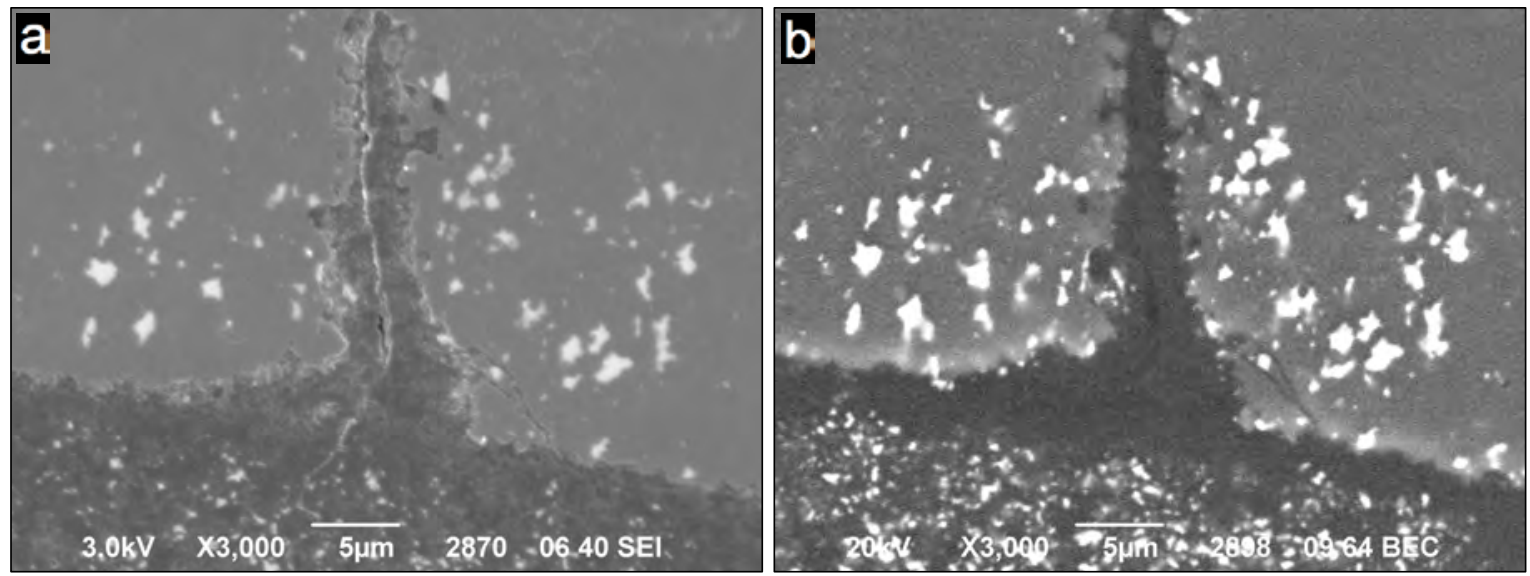

Figure 24. SEM analysis of Particle 442-A053 showing (a) close-up SEI micrograph of degraded region surrounding a $\mathrm{SiC}$ crack filled with a dense material, and (b) close-up BEC micrograph confirming high-Z features flanking the degraded region and low-Z material nearest the crack.
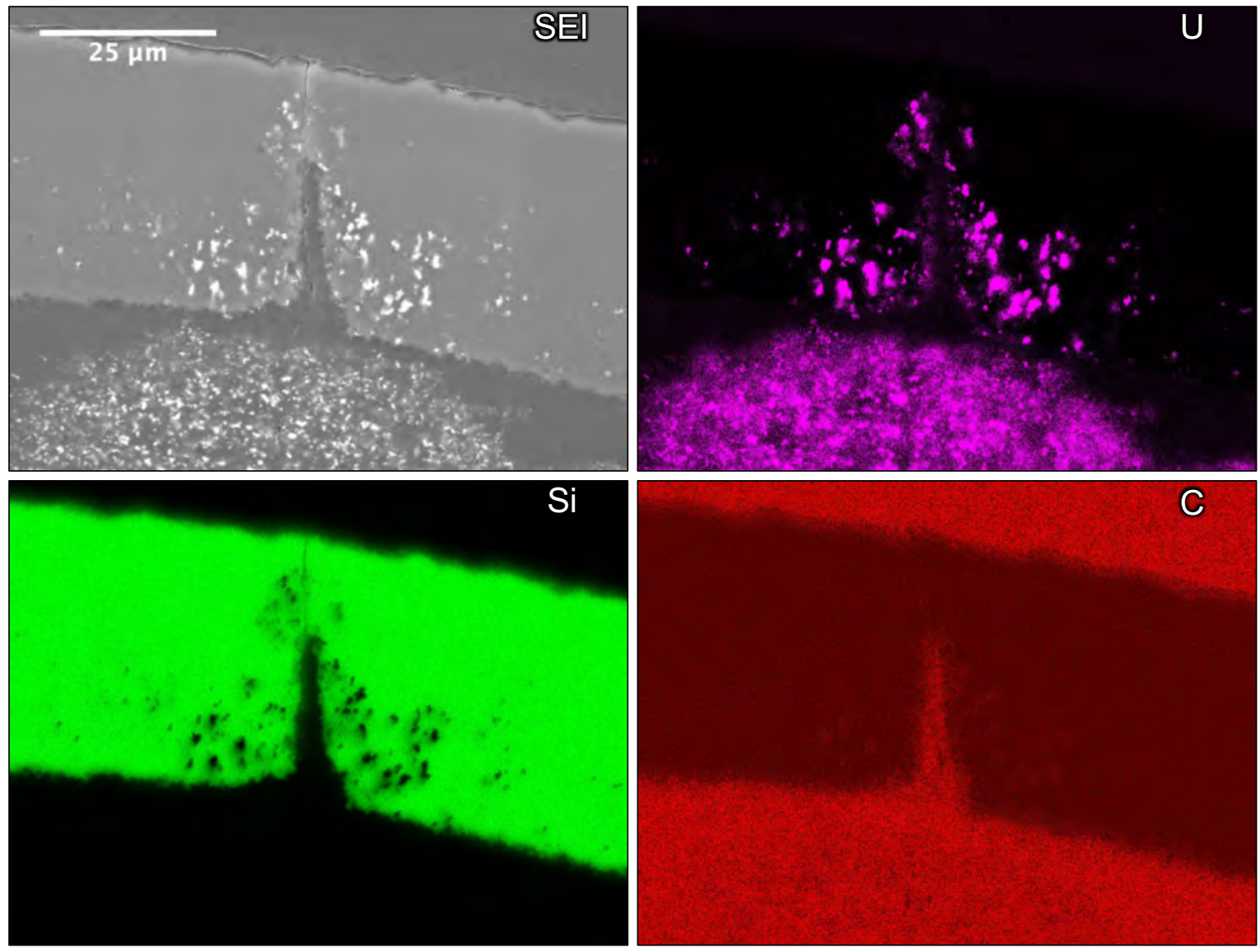

Figure 25. SEM/EDS analysis of Particle 442-A053 showing an overview SEI micrograph of the region surrounding a through-layer crack in the SiC and corresponding EDS x-ray intensity maps indicating relative concentrations of uranium, silicon, and carbon.

As discussed in Section 5.1, Particle 442-A072 experienced kernel protrusion through a gap in the fractured buffer that brought it into contact with the IPyC layer. This led to elevated levels of interaction with the IPyC and SiC layers. Figure 15e is an optical micrograph that shows a region revealed during materialographic sample preparation where the kernel material was intimately in contact with the IPyC; 
an SEI micrograph of this region is shown in Figure 26. In the optical micrograph, the IPyC layer appears to be thinner where it is in contact with the kernel mass. In the SEI micrograph, there is no clear demarcation between the kernel material and the IPyC material. These observations indicate that significant reaction has occurred between these two components. Small white spots throughout the remaining IPyC layer and larger spots in the $\mathrm{SiC}$ layer indicate kernel material (identified below as predominantly uranium) moved into these layers ahead of the primary reaction boundary. Figure $15 \mathrm{e}$ also shows that the $\mathrm{SiC}$ layer appears to be thinner immediately across from the kernel protrusion. Figure 26 shows that the inside surface of the $\mathrm{SiC}$ in this region has been degraded.

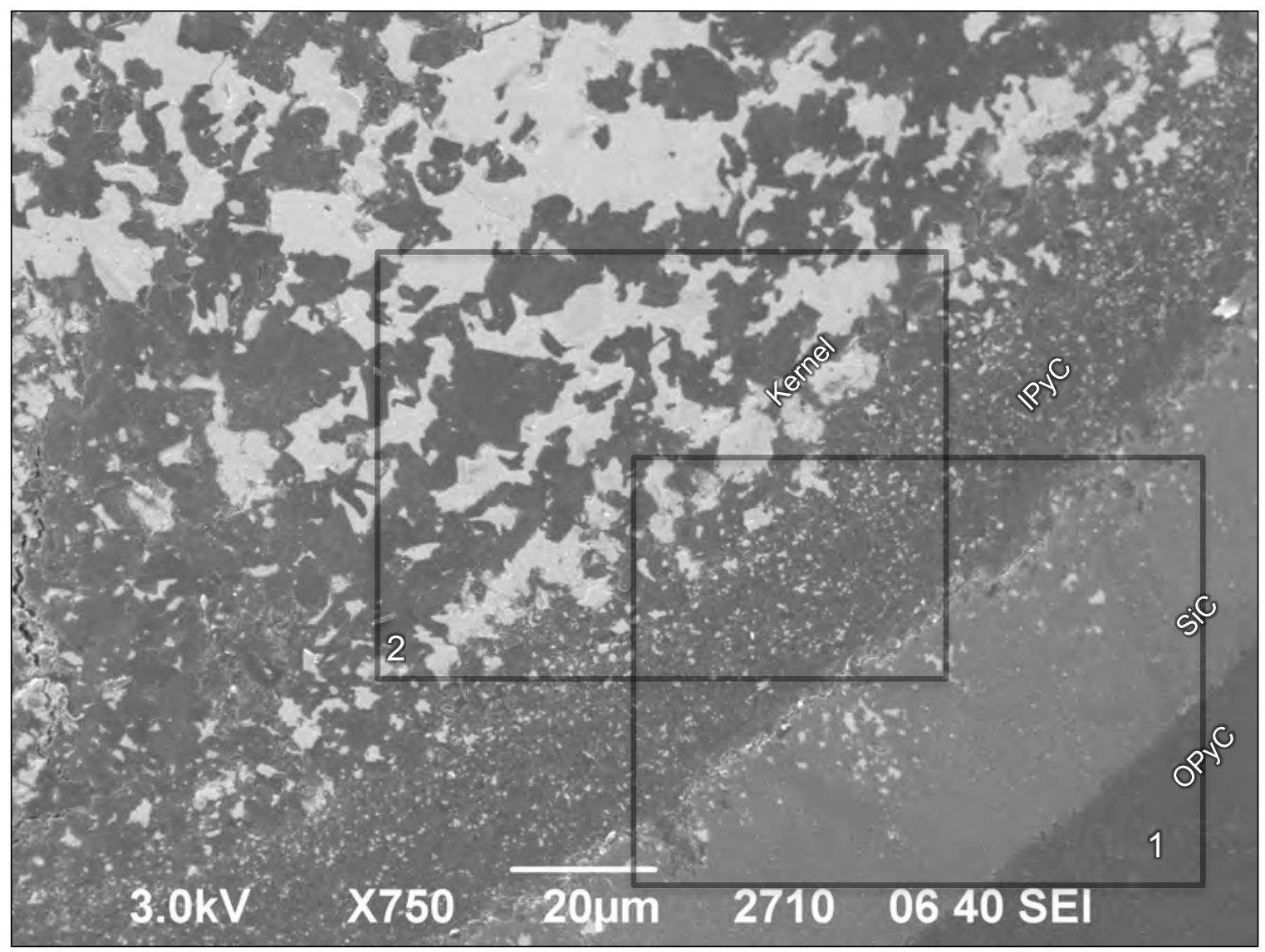

Figure 26. SEI micrograph of Particle 442-A072 showing an area where the kernel protrusion contacted the IPyC layer; the marked zones ( 1 and 2 ) indicate regions investigated by the EDS $x$-ray mapping presented in Figure 27 and Figure 28.

Figure 27 shows an SEI micrograph and associated x-ray intensity maps of Figure 26 Zone 1. Spot analysis was performed on select features to complement the EDS mapping and indicated all high-Z features in the $\mathrm{SiC}$ layer were uranium-rich with two features identified to also contain low concentrations of zirconium and molybdenum (not visible in the x-ray maps). The mapping analysis also showed silicon-rich features inside the IPyC layer that were co-located with carbon, oxygen, uranium, ruthenium and technicium. Fixed-spot analysis also suggested trace concentrations of palladium, rhodium, and plutonium. The precence of these silicon-rich clusters indicates silicon from the SiC layer moved away from the IPyC/SiC interface and was apparently trapped by actinides and fission product species found in the kernel material. 

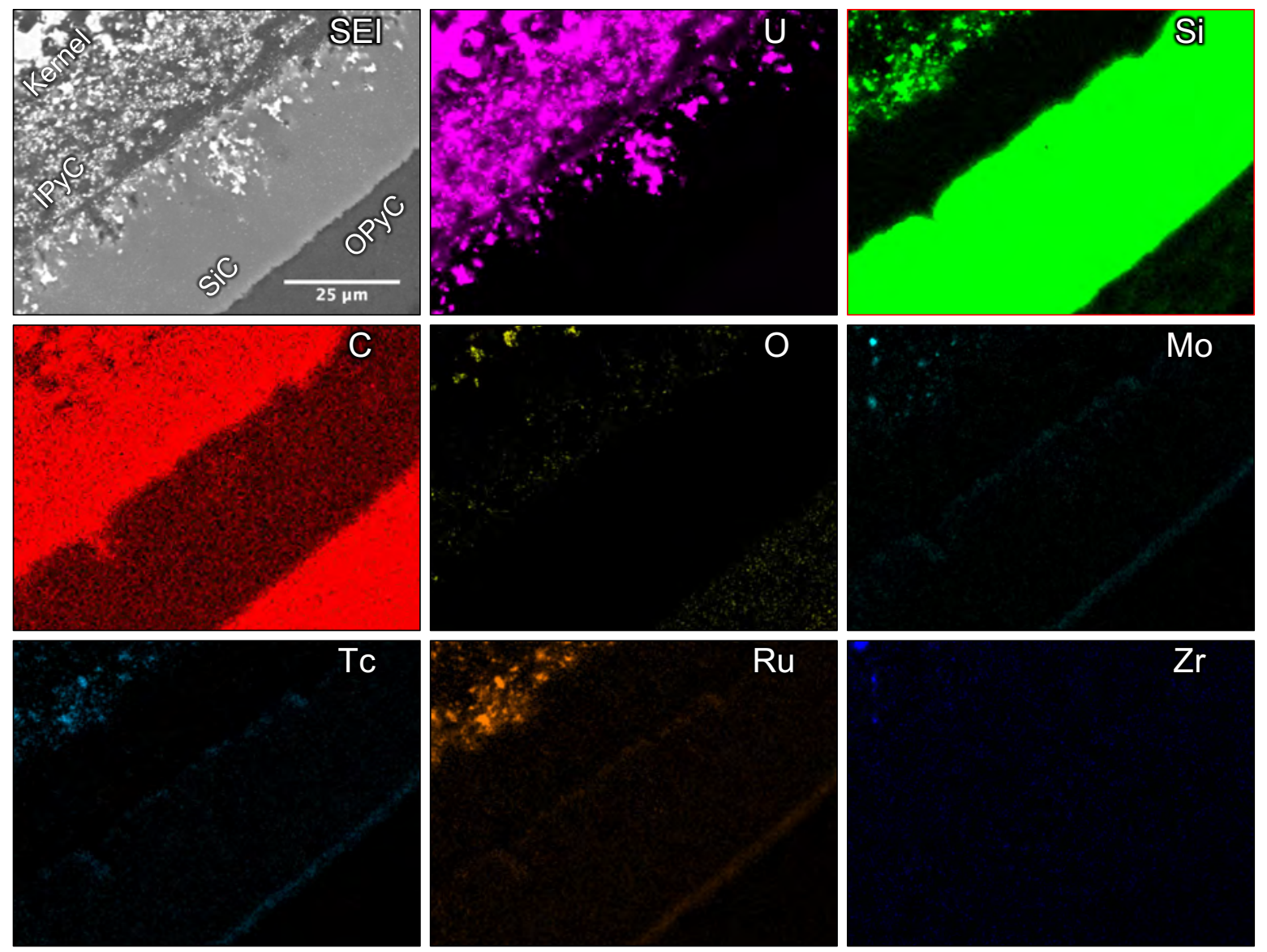

Figure 27. Particle 442-A072 EDS analysis showing SEI micrograph of Figure 26 Zone 1 and corresponding EDS $x$-ray intensity maps indicating relative concentrations of the most prominent elements.

Elemental mapping of Zone 2 was performed to better understand the composition of the protruding kernel material. The analysis shown in Figure 28 confirmed that the silicon-rich phases (also containing carbon, oxygen, uranium, ruthenium, and technetium) were primarily located along the interface of the kernel protrusion and the remaining intact IPyC layer; however, localized features were observed deeper into the kernel protrusion. The analysis also showed large uranium-molybdenum and uranium-zirconium phases with trace technetium concentrations. These phases also contained carbon and oxygen and highlight the ability of the kernel to stabilize and retain primary fission product species. Palladium was identified in a few features by the automatic identification routine in the Oxford software package; however, the peak intensity was too low to confirm its presence.

The SEI micrograph in Figure 26 shows an intertwined network of higher-density material (bright areas) and lower-density material (darker gray areas), with notably little porosity compared to a typical asirradiated kernel [Hunn et al. 2013a]. Some of this structure is visible in the SEI image of Zone 2 (Figure 28). The bright areas in the SEI image align with the uranium map in Figure 28. Where the uranium concentration in these areas was slightly lower, molybdenum and technetium or zirconium were colocated with the uranium. The EDS maps in Figure 28 show that the darker gray areas in the SEI image were mostly carbon. These areas of mostly carbon may be remnants of buffer. The way the metals are interspersed around the carbon areas and the lack of porosity in this region suggests that the metals may have been liquid at some stage. 

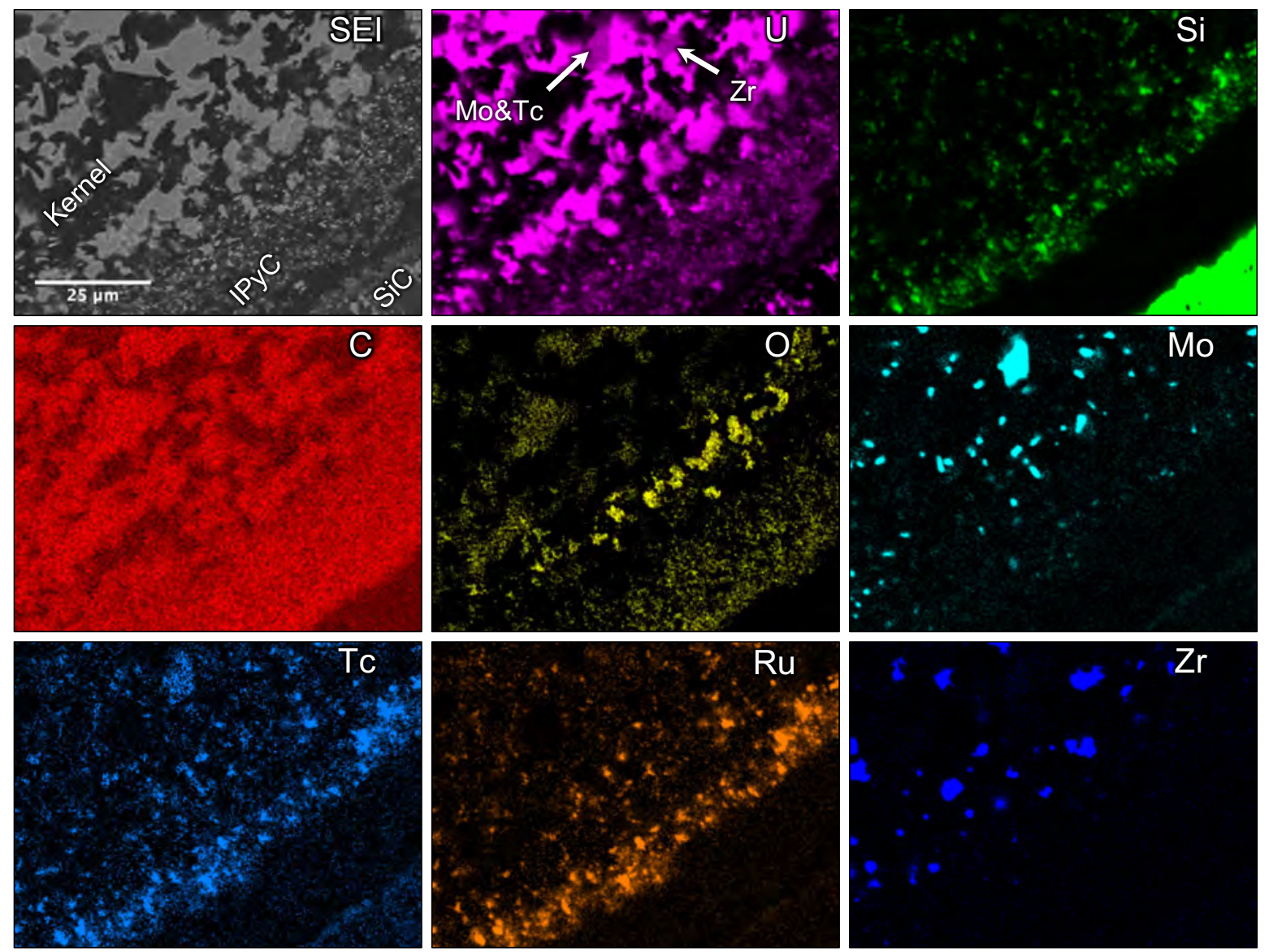

Figure 28. Particle 442-A072 EDS analysis showing SEI micrograph of Figure 26 Zone 2 and corresponding EDS x-ray intensity maps indicating relative concentrations of the most prominent elements.

Figure 29 shows SEM analysis of two regions away from the large kernel protrusion. The BEC micrograph in Figure 29a shows that significant $\mathrm{SiC}$ decomposition occurred where there was a lobeshaped uranium dispersion in the IPyC. Again, the bright spots in this BEC micrograph were determined with EDS to all be uranium-rich and the darker regions in the $\mathrm{SiC}$ were predominantly carbon. In contrast, Figure $29 \mathrm{~b}$ shows different behavior at the IPyC/SiC interface in the absence of concentrated uranium dispersion in the IPyC, where uranium-rich features were only observed near the IPyC/SiC interface.
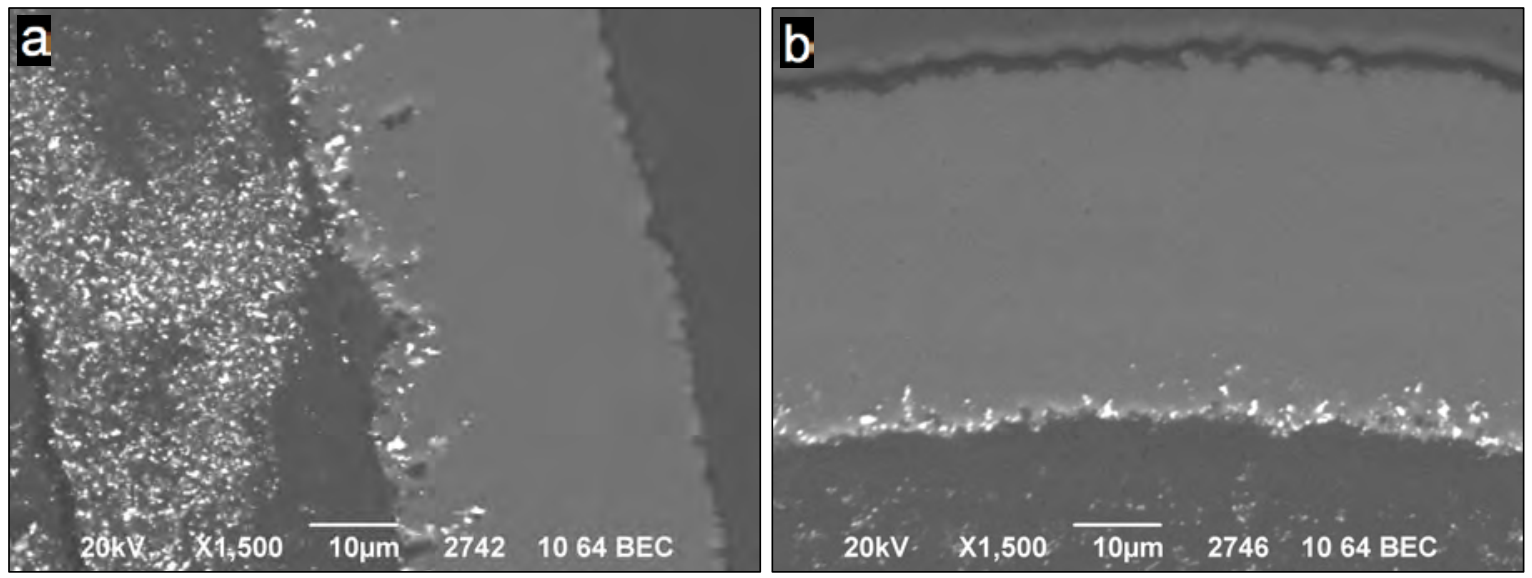

Figure 29. Particle 442-A072 BEC micrographs showing (a) the $\mathrm{SiC}$ layer next to a uranium dispersion lobe with significant $\mathrm{SiC}$ attack and (b) the $\mathrm{SiC}$ layer with no adjacent uranium dispersion lobe in the IPyC layer. 
As discussed in Section 5.1, Particle 442-A050 had a wide channel of degraded SiC at the tip of an IPyC crack and both these features traversed more than half way around the particle (Figure 16c). Preparation of a polished section through Particle 442-A050 revealed a cross section of the channel (Figure 16e), which appeared to be filled with material similar in optical characteristic to the adjacent IPyC in the polished section. Figure 30 shows SEM/EDS analysis of this area. The material inside the channel was carbon-rich and devoid of silicon. Surrounding this feature were large uranium-rich/silicon-poor clusters and carbon-rich/silicon poor spots. Spot analysis with EDS showed no significant intensity from other elements when centered on the uranium-rich features.
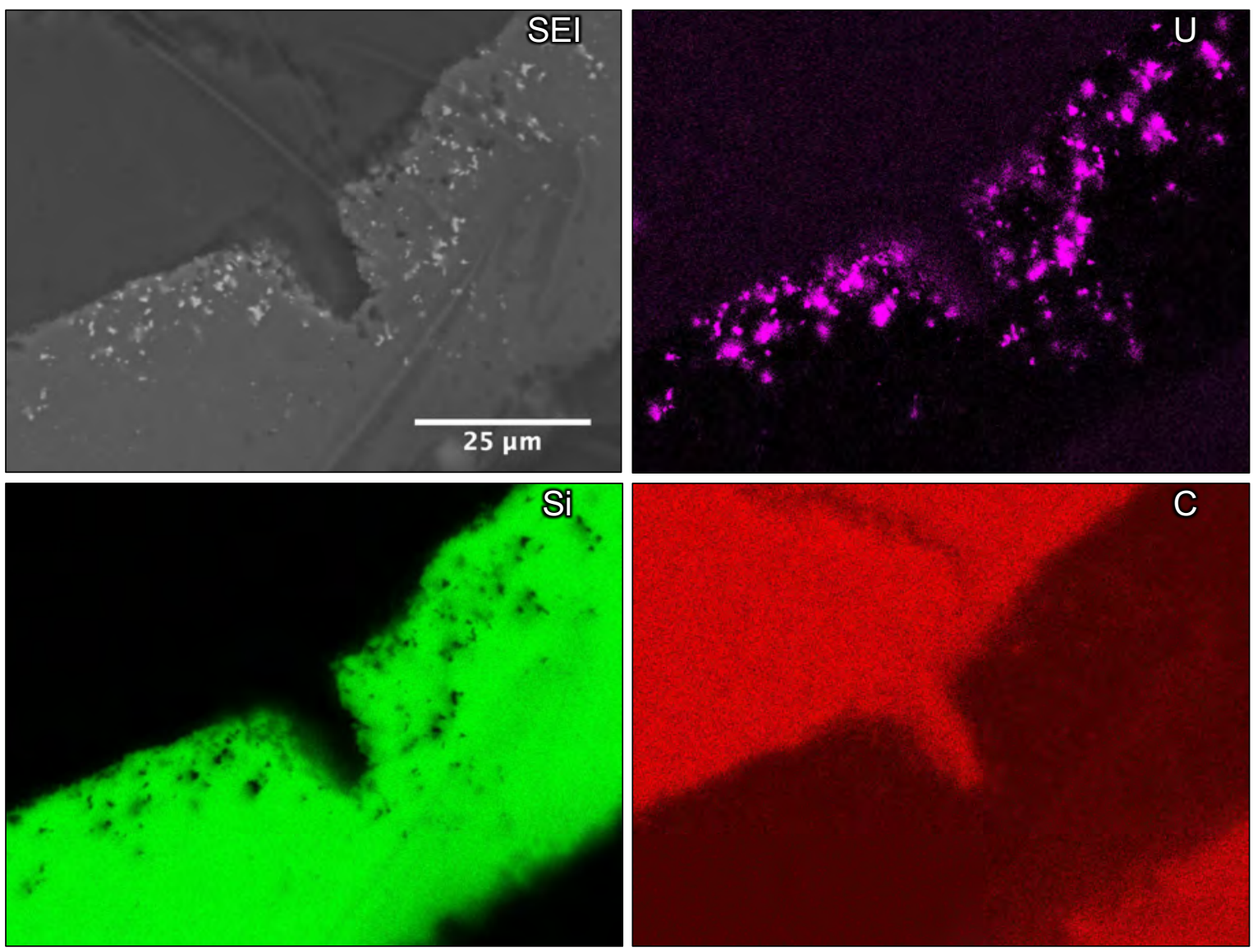

Figure 30. SEM analysis of a SiC decomposition area in Particle 442-A050 showing SEI micrograph and corresponding EDS x-ray intensity maps indicating relative concentrations of uranium, silicon, and carbon. The linear features observed in the SEI micrograph are due to delamination of the $\sim 8$-nm-thick carbon coating applied to reduce charging of the sample during analysis.

Images of two areas in the SiC layer of Particle 442-A050, away from any IPyC cracks, are shown in Figure 31. In general, the SEM analysis showed high-Z features distributed primarily in the first half of the $\mathrm{SiC}$ layer. However, as evident in Figure 31a, features were also present in the outer half of the SiC layer in some locations. The features were confirmed to be uranium-rich and showed no significant EDS signal from other elements. Also evident in Figure 31 are darker spots in the $\mathrm{SiC}$ that were most prevalent in the outer half of the SiC. These were carbon-rich areas and have been observed in particles from other $1800^{\circ} \mathrm{C}$ safety tests. It has been postulated that these spots indicate where clusters of palladium may have resided in the as-irradiated particle prior to heating to $1800^{\circ} \mathrm{C}$ and that the palladium silicide may have diffused out at this temperature [Hunn et al. 2013b, 28]. Similar out-diffusion of palladium silicide has been proposed as an explanation for only observing uranium clusters in the $\mathrm{SiC}$ layers of particles heated to $1800^{\circ} \mathrm{C}$, while as irradiated particles and those safety tested to lower temperature usually exhibit clusters of palladium or palladium colocated with uranium. Figure 31 also shows obvious degradation of 
the $\mathrm{SiC}$ outer surface. This was observed to some extent in all the heated particles and is discussed further in Section 5.3.
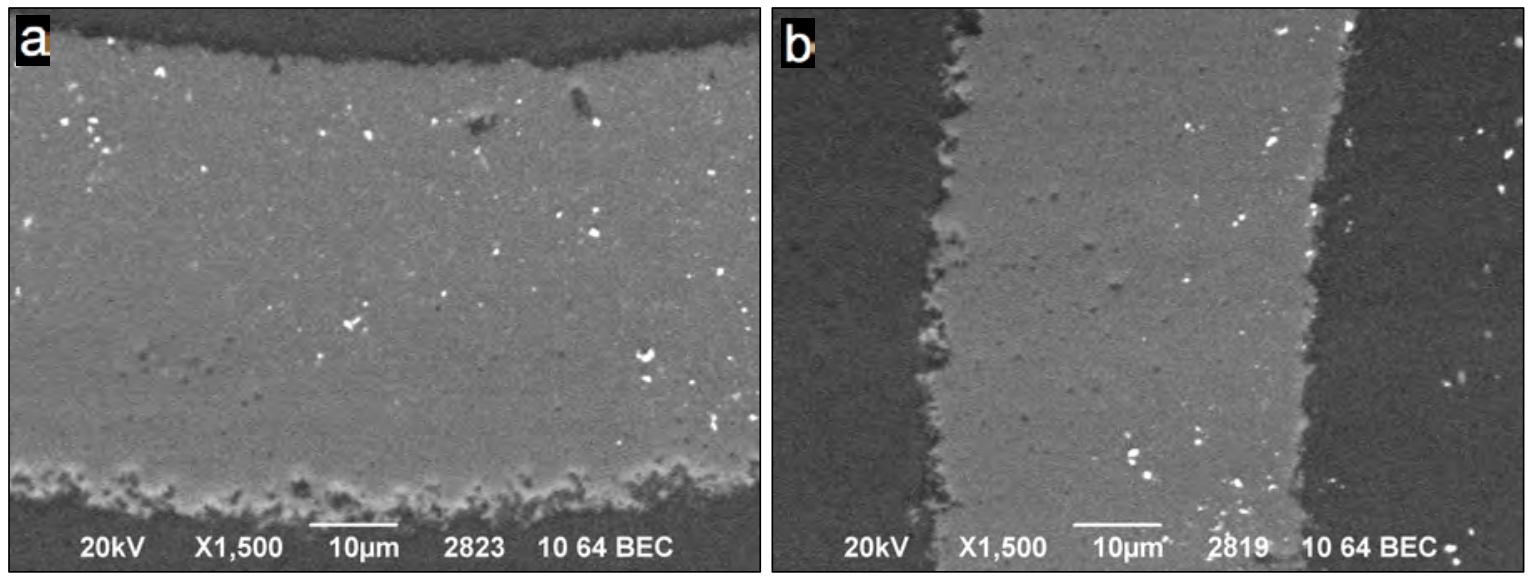

Figure 31. Particle 442-A050 BEC micrographs showing SiC layer not adjacent to a crack in the IPyC layer.

\subsection{Particles that Retained Cesium-Optical Microscopy and SEM with EDS}

Sections 5.1 and 5.2 discussed the post-heating test analysis of the five particles that released cesium due to failed TRISO. The remaining 70 particles all exhibited good cesium retention that is indicative of intact $\mathrm{SiC}$ layers. Particles from this subset were selected for analysis based on their silver and europium retention (Table 7).

Only six particles retained enough silver to measure the ${ }^{110 \mathrm{~m}} \mathrm{Ag}$ activity with IMGA using a six-hour counting time (Table 4). Comparison of the measured ${ }^{110 \mathrm{~m}} \mathrm{Ag}$ activity before and after the test showed that these six particles retained a varied amount of their silver, ranging from approximately $31 \%$ to essentially 100\%-retention in Particle 442-A074 (Table 5). Particle 442-A074 was selected for materialography along with Particle 442-A046, which exhibited the second highest silver retention at $\sim 85 \%$.

Figure 10 and Table 5 show that most of the 70 particles with intact $\mathrm{SiC}$ still released a measurable amount of europium during the heating test. Two particles with the greatest fractional europium release were selected for further analysis; Particle 442-A055 and Particle 442-A059 both exhibited release fractions close to $30 \%$. For comparison, two additional particles were selected that had higher europium retention, Particle 442-A063 and Particle 442-A016. Particle 442-A063 also retained about half its silver, while ${ }^{110 \mathrm{~m}} \mathrm{Ag}$ retention could not be measured in Particle 442-A016. Particle 442-A074 and Particle 442A046, selected for their high silver retention, also retained europium.

Table 7. Description of the six particles investigated by optical microscopy and SEM analysis that retained cesium (as well as ruthenium, antimony, and cerium), but exhibited varied retention of silver or europium.

\begin{tabular}{|c|c|c|c|}
\hline Particle & Description of observed retention & $\begin{array}{c}{ }^{110 m} \mathrm{Ag} \\
\text { Retention } \\
\end{array}$ & $\begin{array}{c}{ }^{154} \mathbf{E u} \\
\text { Retention } \\
\end{array}$ \\
\hline $442-\mathrm{A} 074$ & Highest Ag retention, no measurable release of other isotopes & $109 \%$ & $99 \%$ \\
\hline $442-\mathrm{A} 046$ & Second-highest Ag retention, no measurable release of other isotopes & $85 \%$ & $99 \%$ \\
\hline $442-\mathrm{A} 055$ & Lowest Eu retention, no measurable release of other isotopes except Ag & $<37 \%$ & $72 \%$ \\
\hline $442-\mathrm{A} 059$ & Lowest Eu retention, no measurable release of other isotopes except Ag & $<37 \%$ & $72 \%$ \\
\hline $442-\mathrm{A} 063$ & Moderate Ag retention, no measurable release of other isotopes & $50 \%$ & $101 \%$ \\
\hline $442-\mathrm{A} 016$ & Low Ag retention, no measurable release of other isotopes & $<44 \%$ & $97 \%$ \\
\hline
\end{tabular}


Optical micrographs showing cross-sections of Particle 442-A074 and Particle 442-A016 are shown in Figure 32. Particle 442-A016 exhibited the most typically observed microstructure, namely an intact TRISO layer, buffer shrinkage with near-complete delamination from the IPyC layer, and a slightly friable kernel with a fine-grain structure. Particle 442-A074 had a different and unusual kernel structure. To better understand the kernel variation, EDS x-ray mapping was performed on all kernels. The analysis indicated that the kernel in Particle 442-A074 had a lower carbon to oxygen ratio than all other analyzed kernels. This suggests the Particle 442-A074 kernel initially had relatively more uranium oxide versus uranium carbide compared to the typical AGR-1 kernel. The enhanced retention during heating may have been due to the higher oxide content and denser kernel structure.
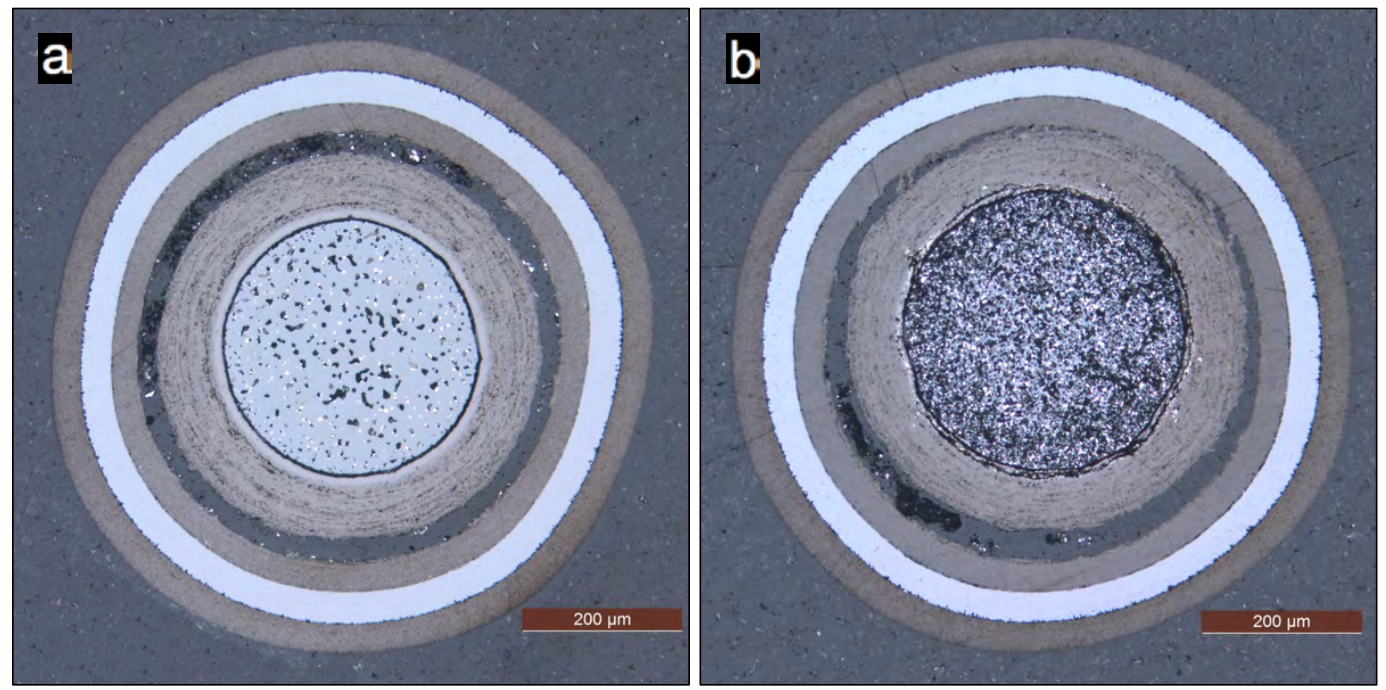

Figure 32. Optical micrographs of particles that retained cesium: (a) Particle 442-A074 with high silver retention, and (b) Particle 442-A016 with low silver retention.

The SEM analysis of the particles in Table 7 focused on microstructural variations and changes in the fission product distribution in the TRISO layers. Figure 33 shows two SEI/BEC image pairs acquired at one location on the polished cross section of Particle 442-A063. Distinct features are labeled in Figure 33 that were observed to some extent in all the particles analyzed in this study. With the exception of the degraded $\mathrm{SiC}$ at the $\mathrm{OPyC} / \mathrm{SiC}$ interface (also shown in Figure 34), these features have been previously observed in other $1800^{\circ} \mathrm{C}$ safety-tested particles [Hunn et al. 2014e]. As already discussed in Section 5.2 with regard to the SEM analysis of the five failed-TRISO particles, bright spots in the BEC micrographs indicate clusters of high-Z elements, and these were observed finely scattered throughout the IPyC layer, embedded in the $\mathrm{SiC}$ in larger clusters (especially in the inner half of the layer), and piled up at the $\mathrm{IPyC} / \mathrm{SiC}$ interface. In the outer half of the $\mathrm{SiC}$ layer, dark spots indicate low-density volumes that have been previously studied in other samples [Hunn et al. 2013b] and appear to be residual carbon-rich features left behind when palladium exited the layer. Degradation of the outer surface of the SiC layer was also observed in the particles with failed TRISO (e.g., Figure 31). This degradation was observed around the circumference of each particle cross-section, for all particles analyzed. Spot analysis with EDS indicated the degraded region was primarily silicon and carbon with low concentrations of oxygen, and the region was depleted of silicon relative to an unaffected reference volume in the $\mathrm{SiC}$ bulk. The nature of the degraded region suggests that thermal decomposition of the $\mathrm{SiC}$ layer may have occurred, followed by transport of silicon away from the $\mathrm{SiC} / \mathrm{OPyC}$ interface. However, this feature was not observed in previous $1800^{\circ} \mathrm{C}$ safety tests. Multiple variables may have contributed to the observed $\mathrm{SiC} / \mathrm{OPyC}$ degradation. Firstly, the loose particle testing was carried out for $600 \mathrm{~h}$ compared to $300 \mathrm{~h}$ for previous tests and the longer exposure may have led to observable decomposition. Secondly, the particles were subjected to deconsolidation prior to the heating test and the lack of surrounding matrix may have influenced the high-temperature decomposition behavior of the $\mathrm{SiC}$ layer. 

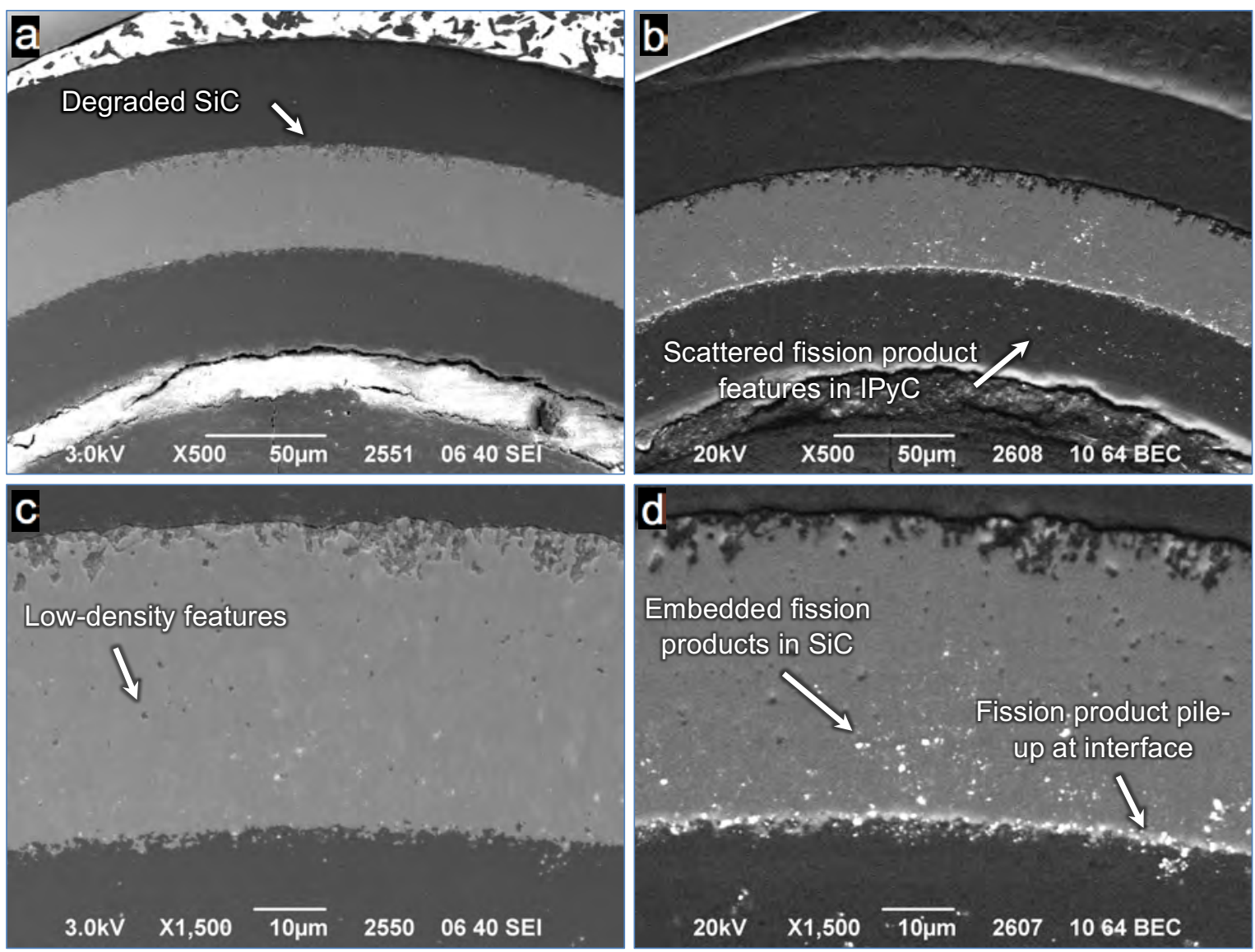

Figure 33. Particle 442-A063 SEM analysis showing (a and c) SEI versus (b and d) BEC micrographs at two magnifications; some typically-observed features are identified.

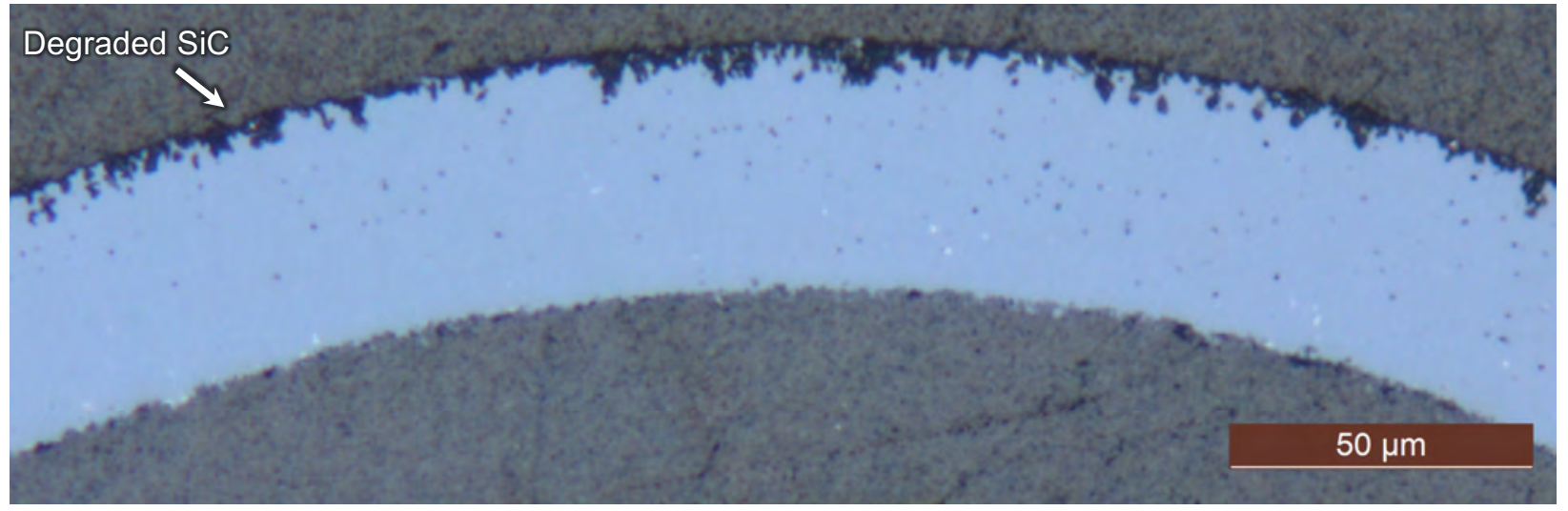

Figure 34. Optical micrograph of Particle 442-A063 showing SiC degradation at OPyC/SiC interface.

The distribution of high- $\mathrm{Z}$ features in the $\mathrm{SiC}$ layer varied qualitatively as a function of ${ }^{110 \mathrm{~m}} \mathrm{Ag}$ retention. Figure 35 compares BEC micrographs of all six particles in Table 7. Particle 442-A074 showed almost no high- $\mathrm{Z}$ features in the $\mathrm{SiC}$ and limited high-Z pileup at the $\mathrm{IPyC} / \mathrm{SiC}$ interface. Particle $442-\mathrm{A} 046$ and Particle 442-A063, which still retained measurable inventories of ${ }^{110 \mathrm{~m}} \mathrm{Ag}$ appeared to have the majority of their high-Z features limited to within $10 \mu \mathrm{m}$ from the IPyC/SiC interface. The particles that released the most ${ }^{110 \mathrm{~m}} \mathrm{Ag}$ had a higher frequency of high- $\mathrm{Z}$ features in the $\mathrm{SiC}$, and these were distributed across the entire $\mathrm{SiC}$ layer. Local $\mathrm{SiC}$ degradation at the $\mathrm{IPyC} / \mathrm{SiC}$ interface was also observed for Particle 442-A055 and Particle 442-A059. An example of this local attack is shown in Figure 36. 

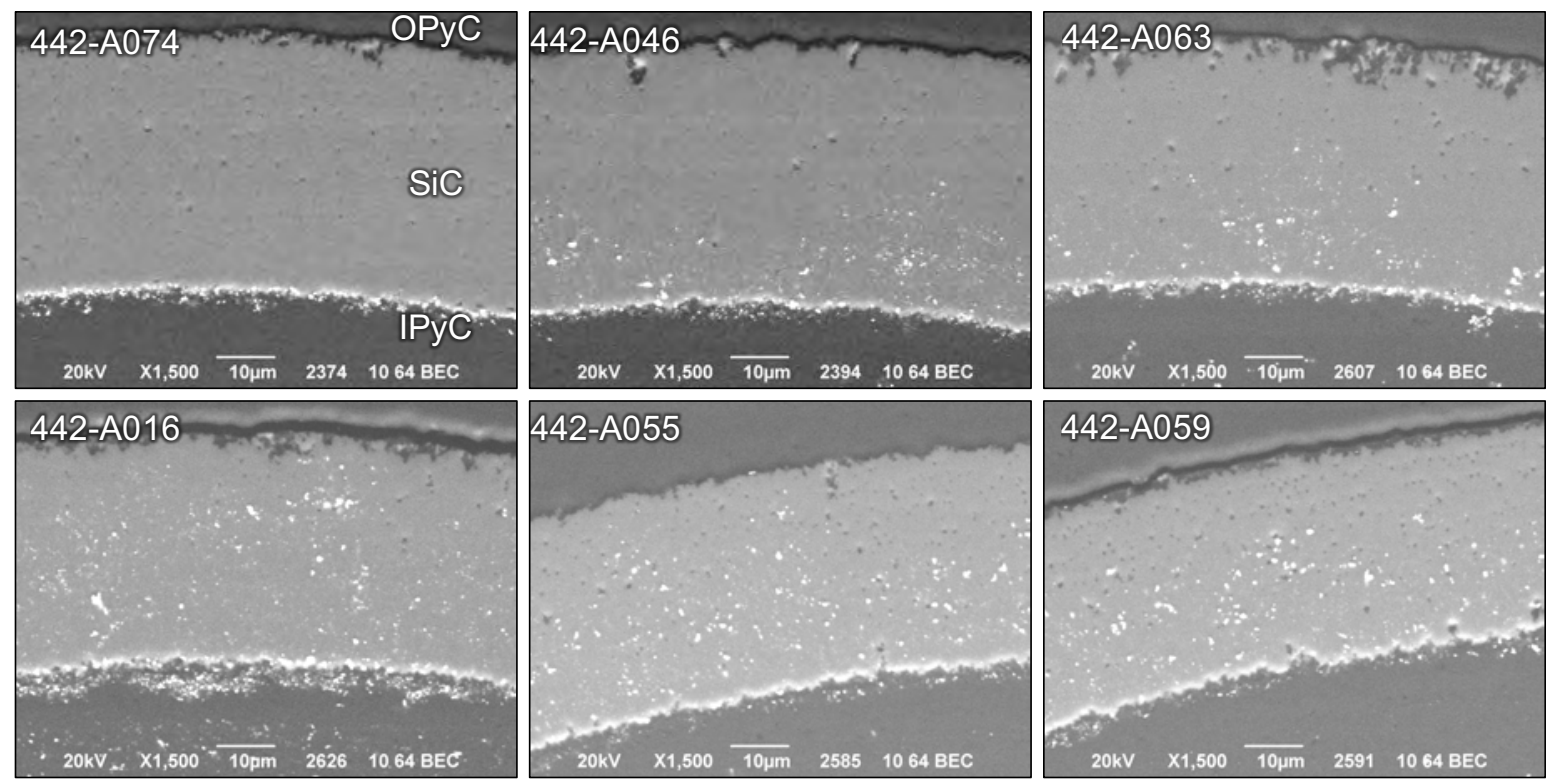

Figure 35. Comparison of BEC micrographs of TRISO layers from particles in Table 7 highlighting the interaction of fission products with the $\mathrm{SiC}$ as a function of silver retention.

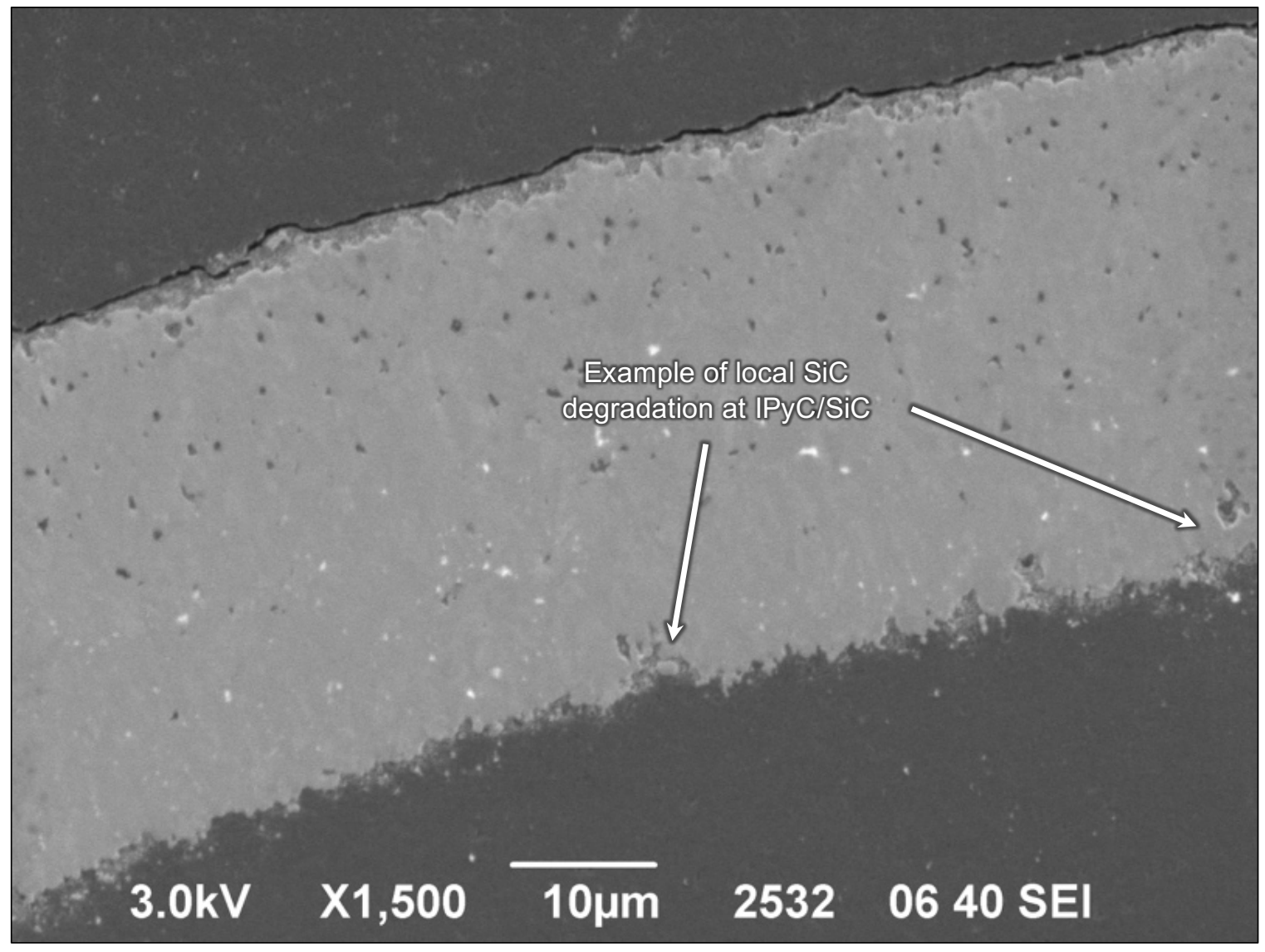

Figure 36. SEI micrograph of Particle 442-A055 showing regions of SiC degradation.

The compositions of the fission product features observed in the IPyC and SiC layers were investigated by EDS analysis that included both fixed-spot analysis and $\mathrm{x}$-ray mapping. The fission product distribution and composition of Particle 442-A074 was distinctly different from the other five particles in 
Table 7, presumably due to the low carbon content in the kernel. As noted earlier, the features were primarily isolated in the IPyC layer and at the IPyC/SiC interface. A compositional EDS map of the $\mathrm{SiC}$ layer of Particle 442 A074 is shown in Figure 37, the mapping indicates pileup of palladium, rhodium, and ruthenium at the $\mathrm{IPyC} / \mathrm{SiC}$ interface with trace uranium concentrations observed in the IPyC layer. Fixed-spot analysis provides improved compositional sensitivity and was employed to better understand the nature of the observed features. The composition of the features at the IPyC/SiC interface were confirmed to be palladium, rhodium, and ruthenium. Trace technetium and molybdenum concentrations were also identified in these features as was trace uranium in select cases. The features were mostly palladium-rich, however, multiple analyzed features were observed to be rhodium or ruthenium-rich. A few fission product features located in the $\mathrm{SiC}$ layer close to the IPyC were primarily palladium, with trace rhodium and ruthenium.
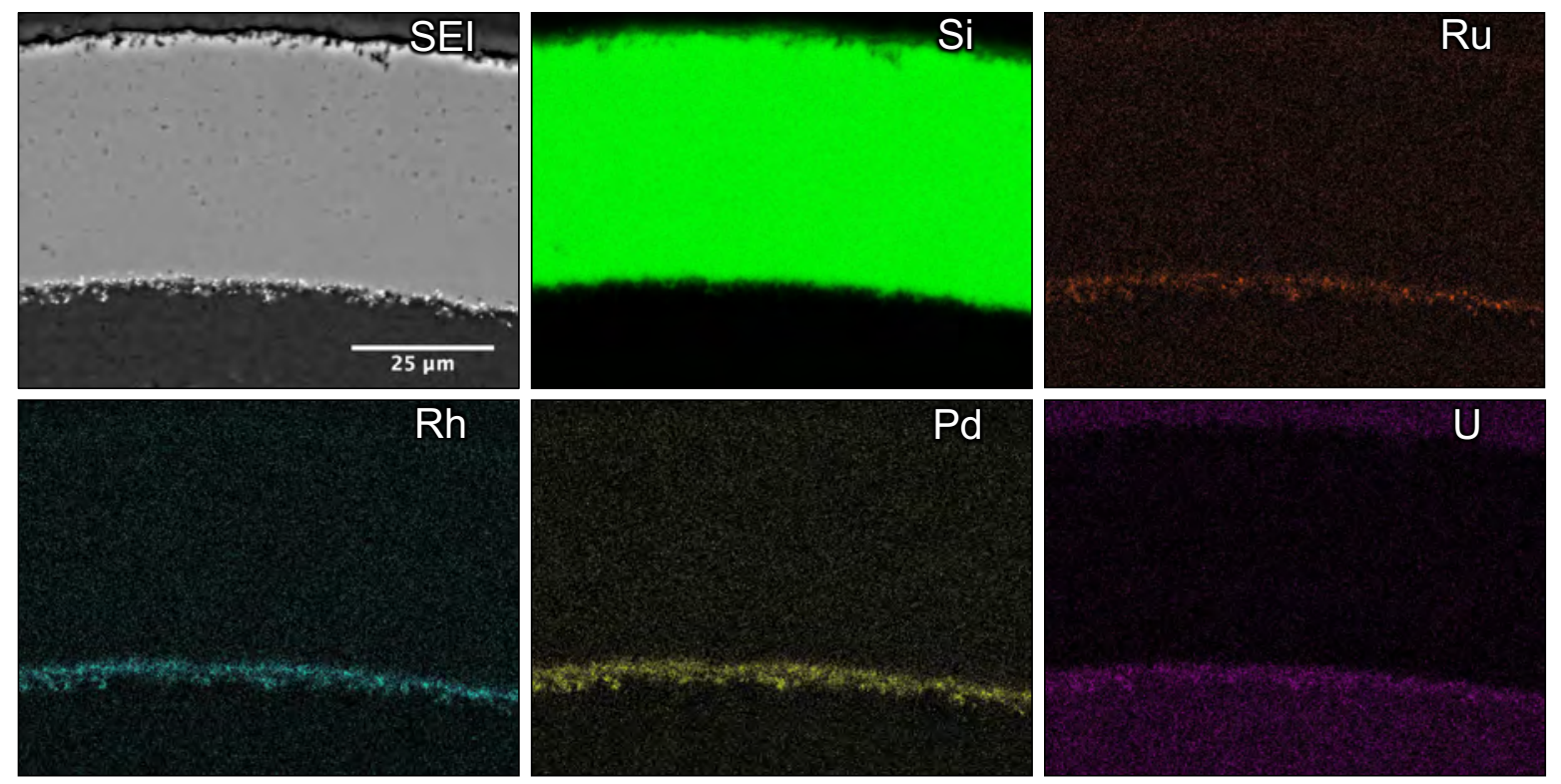

Figure 37. SEM/EDS analysis of IPyC and SiC layers in Particle 442-A074 showing SEI micrograph and corresponding EDS x-ray intensity maps of relative silicon, ruthenium, rhodium, palladium, and uranium concentrations.

The analysis of the other five particles with normal cesium retention indicated four common feature types; these common features were uranium-only (U-only) features, uranium with relatively-low concentration palladium (U-dominant) features, uranium molybdenum zirconium (U-Mo-Zr) features, and complex palladium, uranium, rhodium, and ruthenium (Pd-U-Rh-Ru) features. Both the U-Mo-Zr and the $\mathrm{Pd}-\mathrm{U}-\mathrm{Rh}-\mathrm{Ru}$ features had varying relative compositions based on the particle of interest and location of the feature. Trace concentrations of $\mathrm{Nd}, \mathrm{Ce}$, and $\mathrm{Pu}$ were also identified by the Oxford automatic identification software routine, however the intensity of the representative $\mathrm{x}$-ray lines for each species were not statistically significant or could not be confirmed due to peak overlap with primary components. The four feature types were observed at varying degrees in all the particles in Table 7 but Particle A074. Figure 38 through Figure 42 show the EDS x-ray maps of the SiC layer for these five particles. The EDS maps visually indicate the difference in fission product distribution at the IPyC/SiC interface and in the $\mathrm{SiC}$ layer. The mapping shows that the uranium-rich features were distributed within the $\mathrm{SiC}$ layer and at the $\mathrm{IPyC} / \mathrm{SiC}$ interface. The intensity of palladium, rhodium, and ruthenium signals were generally concentrated at the IPyC/SiC interface, but lower intensities were also observed in the $\mathrm{SiC}$ layer, especially for palladium. 

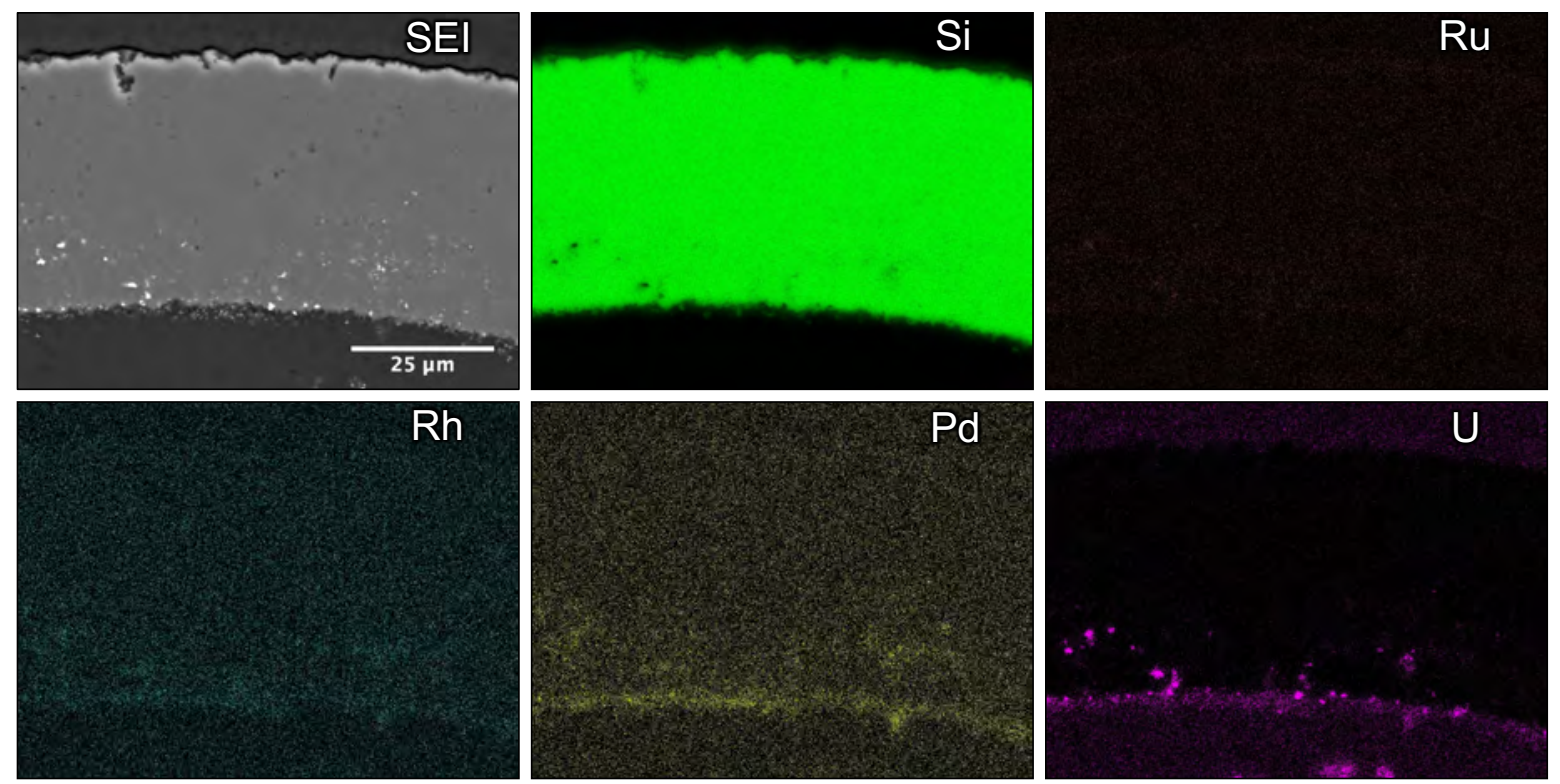

Figure 38. SEM/EDS analysis of IPyC and SiC layers in Particle 442-A046 showing SEI micrograph and corresponding EDS $x$-ray intensity maps of relative silicon, ruthenium, rhodium, palladium, and uranium concentrations.
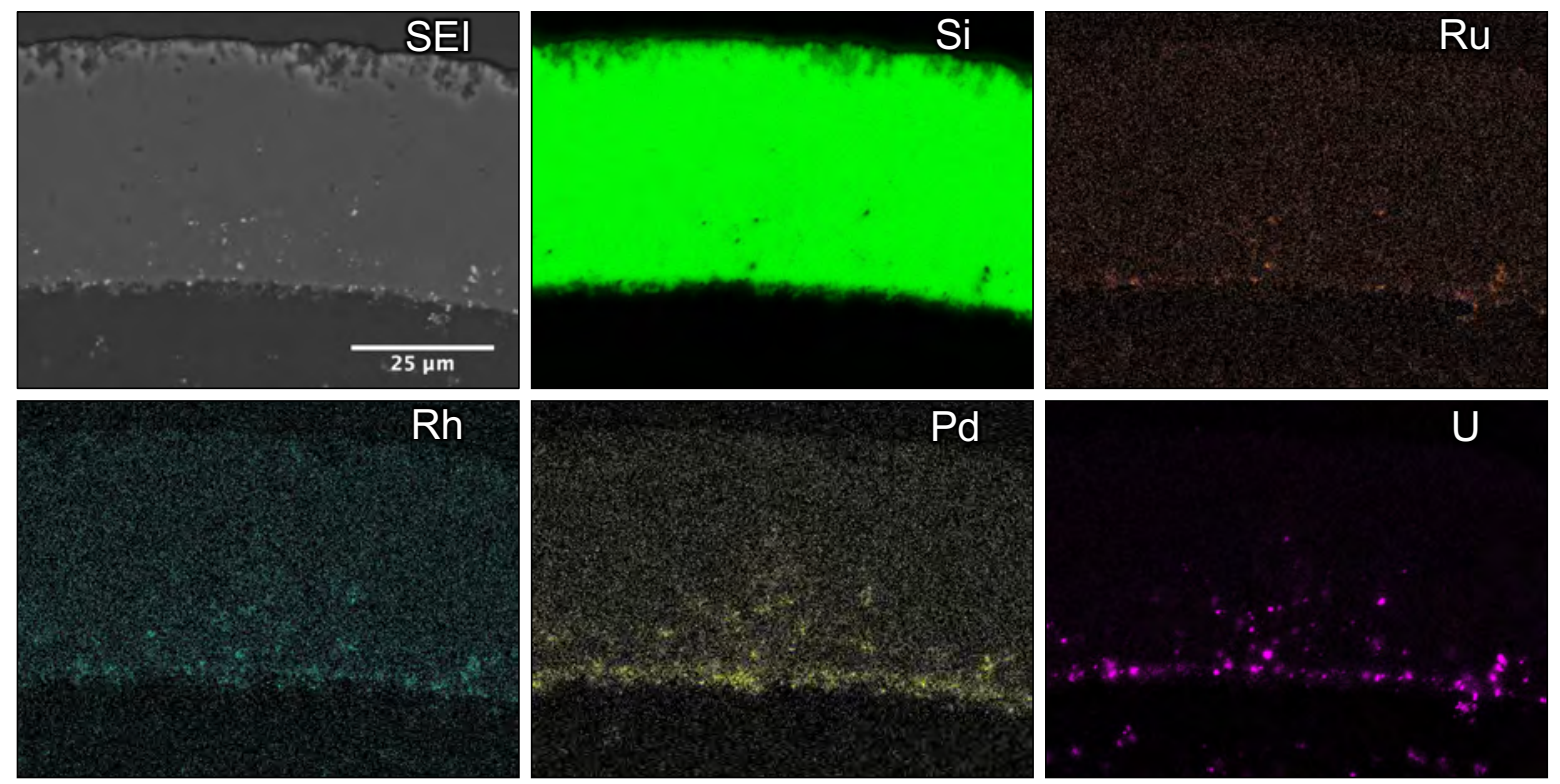

Figure 39. SEM/EDS analysis of IPyC and SiC layers in Particle 442-A063 showing SEI micrograph and corresponding EDS $x$-ray intensity maps of relative silicon, ruthenium, rhodium, palladium, and uranium concentrations. 

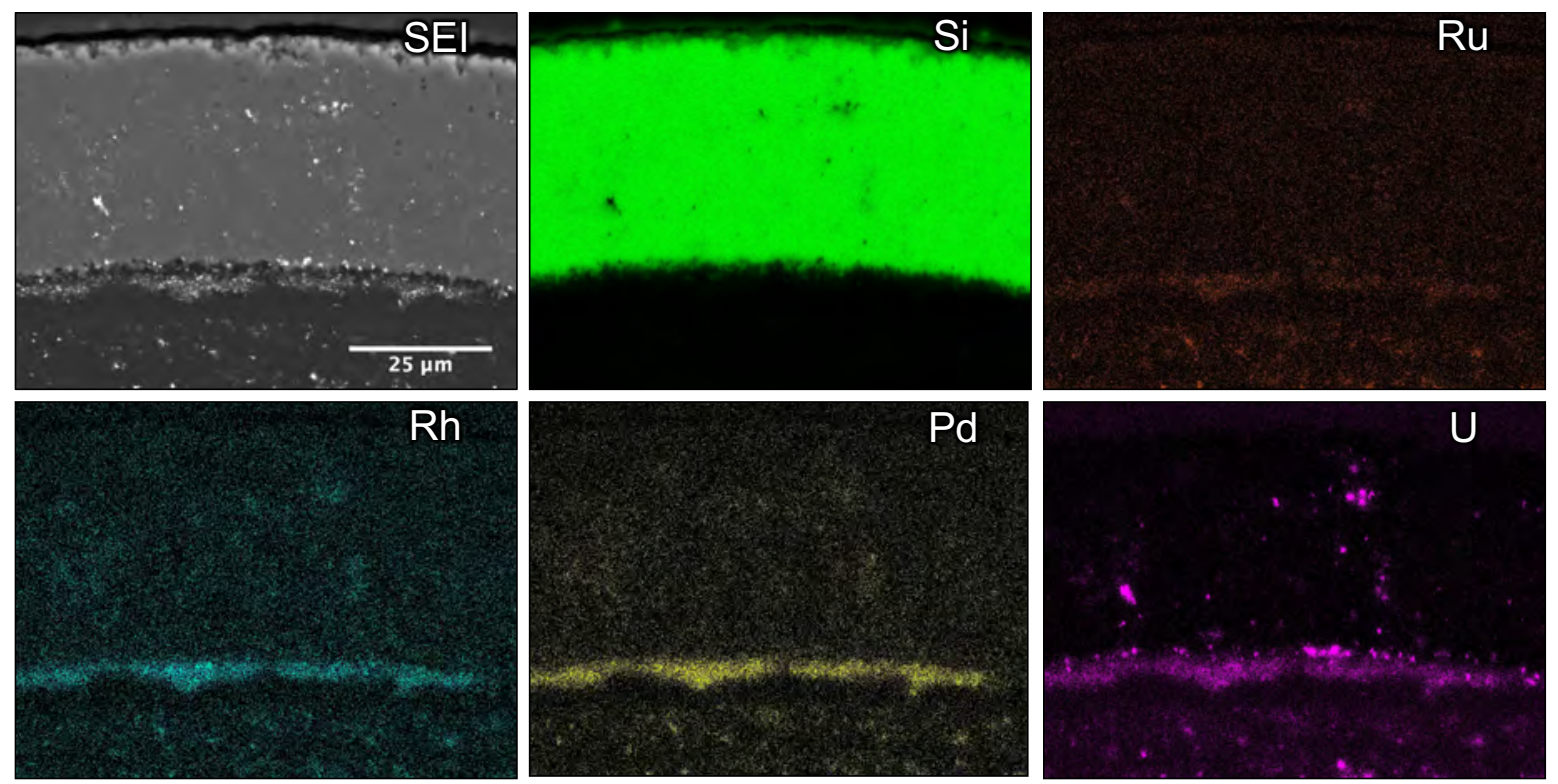

Figure 40. SEM/EDS analysis of IPyC and SiC layers in Particle 442-A016 showing SEI micrograph and corresponding EDS $x$-ray intensity maps of relative silicon, ruthenium, rhodium, palladium, and uranium concentrations.
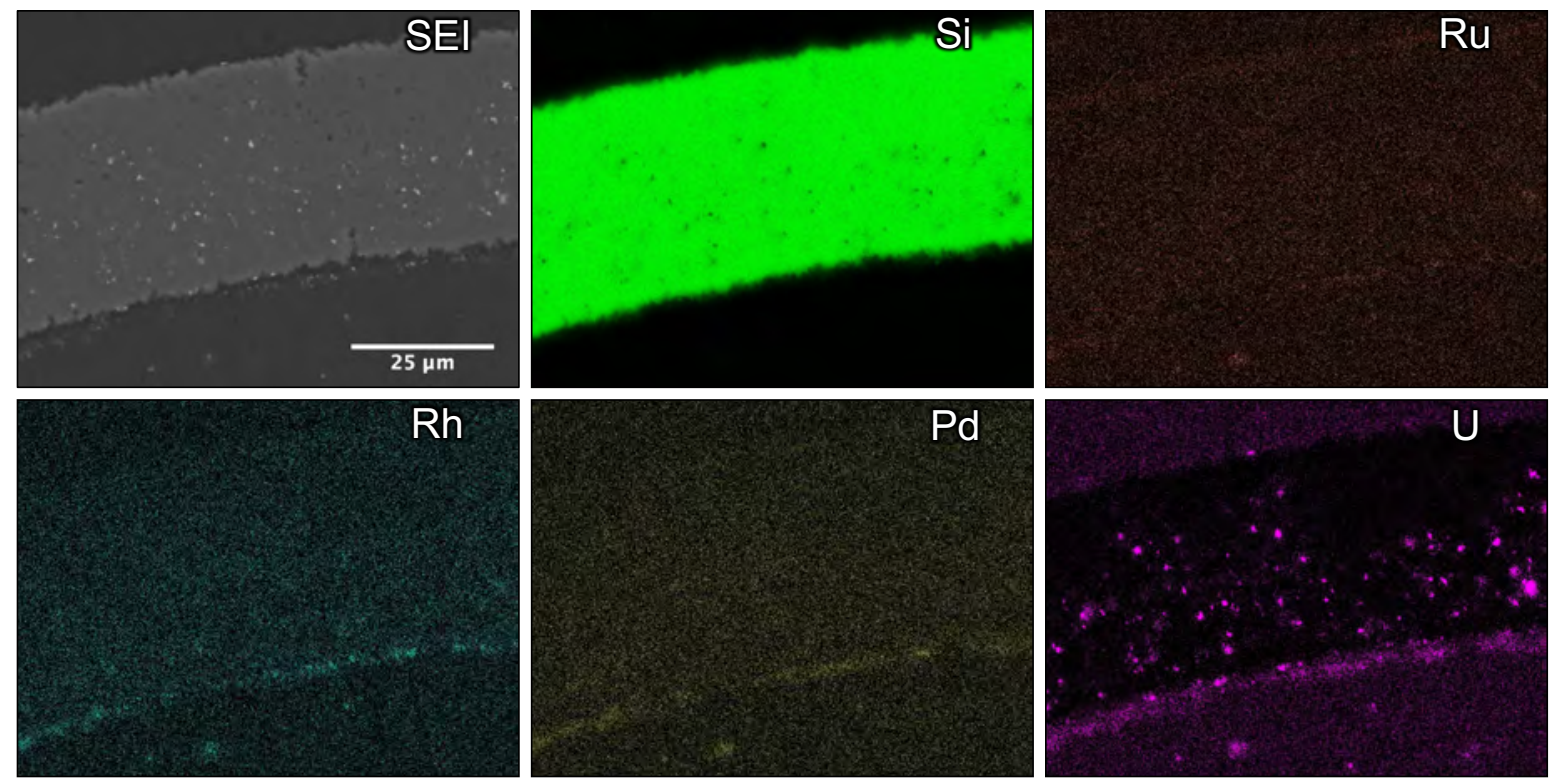

Figure 41. SEM/EDS analysis of IPyC and SiC layers in Particle 442-A055 showing SEI micrograph and corresponding EDS $x$-ray intensity maps of relative silicon, ruthenium, rhodium, palladium, and uranium concentrations. 

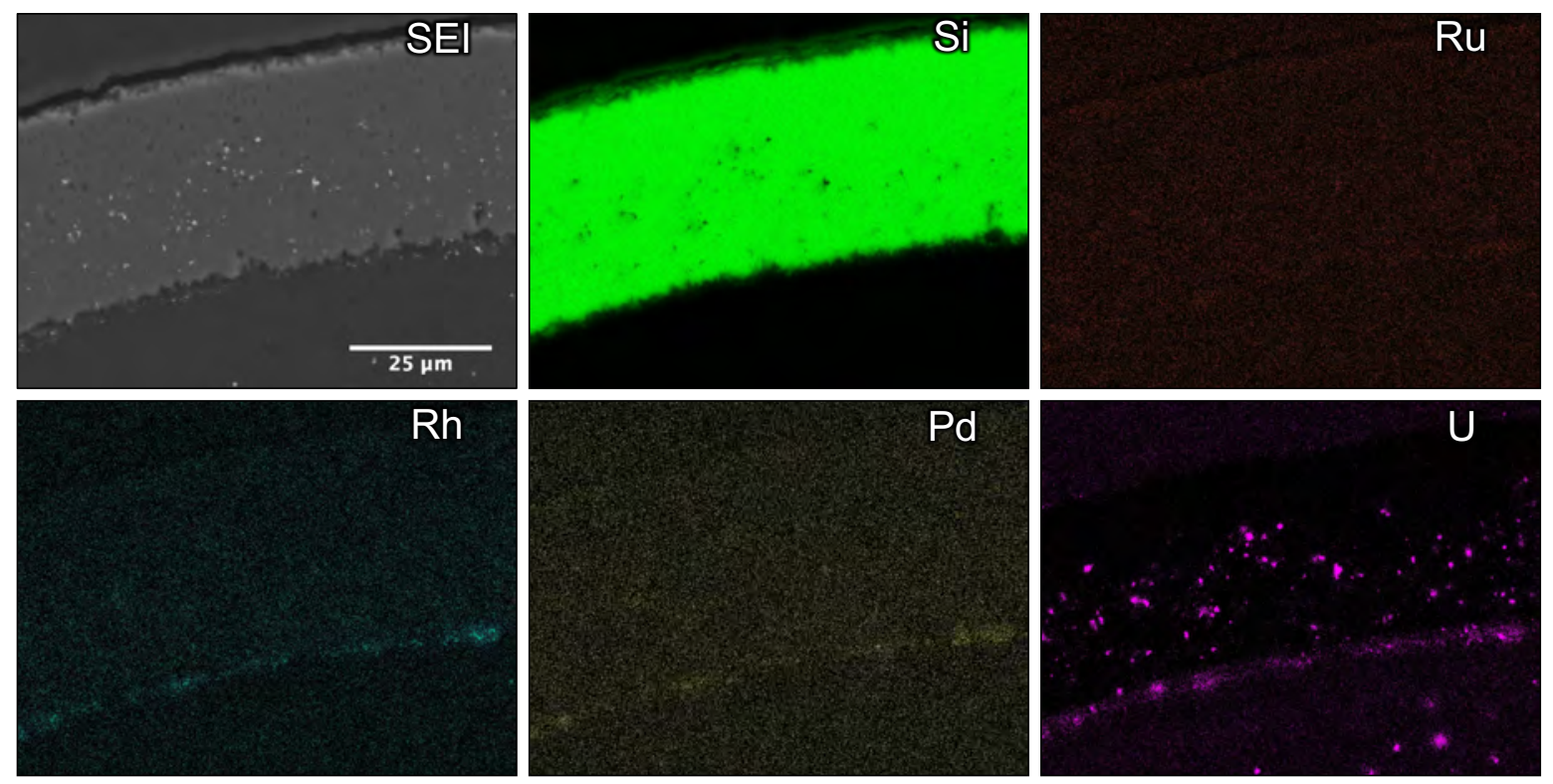

Figure 42. SEM/EDS analysis of IPyC and SiC layers in Particle 442-A059 showing SEI micrograph and corresponding EDS x-ray intensity maps of relative silicon, ruthenium, rhodium, palladium, and uranium concentrations.

Fixed-spot analysis was performed to better understand the fission product and uranium interactions with the $\mathrm{SiC}$ layer. Some similarities were observed when comparing the fission product behavior among the particles. Particles 442-A046, 442-A063, and 442-A016 showed similar trends in feature compositions. The majority of features at the IPyC/SiC interface were $\mathrm{Pd}-\mathrm{U}-\mathrm{Rh}-\mathrm{Ru}$ complexes and palladium-rich. The majority $(\sim 70-80 \%)$ of the features in the $\mathrm{SiC}$ layer were U-rich features (i.e., U-dominant with trace $\mathrm{Pd}$, U-only, or U-Mo-Zr features), while the other $\sim 20-30 \%$ of the features were Pd-U-Rh-Ru features. A unique fission product feature composition of U-Mo-Tc was observed in particle 442-A063. The fission product distribution observed in Particles 442-A059 and 442-A055 showed notably different behavior from the other grouping. In Particles 442-A059 and 442-A055, the features at the IPyC/SiC interface had slightly more uranium than palladium with either equivalent or higher rhodium concentration, this varied from the palladium-rich nature of these IPyC/SiC interface features in Particles 442-A046, 442-A063, and 442-A016. Almost all of the features analyzed in the Particles 442-A059 and 442-A055 SiC layers were U-only or U-Mo-Zr features, with only $4 \%$ and $8 \%$ of the analyzed features in Particle 442-A059 and Particle 442-A055 having the U-Mo-Zr composition, respectively.

The different fission product distributions and compositions in Particles 442-A059 and 442-A055 reflect the different silver and europium retention behavior of these two particles, compared to the other particles in Table 7. The uranium was distributed further out in the $\mathrm{SiC}$ than in Particles 442-A046 and 442-A063 and, as discussed with regard to Figure 35 above, this trend correlated to the silver retention and was also seen in Particle-A016, which also released most of its silver. However, in contrast to Particle-A016 that did not release measurable europium, Particles 442-A046 and 442-A063 also showed less palladium remaining in the $\mathrm{SiC}$ or at the $\mathrm{IPyC} / \mathrm{SiC}$ interface. 


\section{CONCLUSION}

Seventy-five individual TRISO particles deconsolidated from AGR-1 Compact 4-4-2 were heated in the ORNL CCCTF to $1800^{\circ} \mathrm{C}$ for $\sim 650 \mathrm{~h}$ in a special graphite tray assembly (Figure 1). Release of certain gaseous and metallic fission products $\left({ }^{85} \mathrm{Kr},{ }^{90} \mathrm{Sr},{ }^{110 \mathrm{~m}} \mathrm{Ag},{ }^{134} \mathrm{Cs},{ }^{137} \mathrm{Cs},{ }^{144} \mathrm{Ce},{ }^{154} \mathrm{Eu}\right.$, and $\left.{ }^{155} \mathrm{Eu}\right)$ was monitored during the test. Krypton release in the CCCTF indicated that five particles experienced failure of all three gas-retentive TRISO layers. Concurrent cesium release was also observed, confirming this TRISO failure. Measurement of the gamma-emitting isotopic inventory within each particle with the IMGA easily identified the five particles with failed TRISO from their very-low inventories of cesium and europium (Figure 10). No SiC failure was evident in the remaining 70 particles.

Krypton release from failed TRISO is an unusual result for AGR-1 safety testing. None of the other thirteen CCCTF tests has resulted in TRISO failure and only diffusive releases of $<25 \%$ of one particle's

${ }^{85} \mathrm{Kr}$ inventory from multiple particles with $\mathrm{SiC}$ failure has been observed at $1800^{\circ} \mathrm{C}$ [Morris et al. 2014]. The one $1800^{\circ} \mathrm{C}$ AGR-1 compact safety test in the INL FACS furnace resulted in failed TRISO with similar ${ }^{85} \mathrm{Kr}$ release kinetics to those observed in this loose particle test. However, the two suspected failed-TRISO particles were not recovered for further analysis, so details of the failure mechanism in the FACS test are not known [Demkowicz et al. 2015a]. Based on the relatively high TRISO failure fraction in the Compact 4-4-2 loose particle test (5/75), it is presumed that the failure was related to deconsolidation of the particles from their surrounding matrix.

Removing particles from the encompassing compact matrix and heating to $1800^{\circ} \mathrm{C}$ as loose particles resulted in significantly-different post-safety test microstructures than were observed after any of the other CCCTF tests [Demkowicz et al. 2015b]. Typical SiC failure in AGR-1 particles tested in the CCCTF at $1700-1800^{\circ} \mathrm{C}$ has involved localized degradation of the $\mathrm{SiC}$ at the tip of an IPyC fracture that produced a carbon-filled pathway through otherwise undamaged $\mathrm{SiC}$. The key difference in the failedTRISO particles from the loose particle test was that these particles experienced SiC fracture over an extended arc, which apparently led to, or was coupled with, OPyC failure.

It has been previously observed that deconsolidation from the compact matrix can lead to OPyC fracture. During irradiation, a gap develops between the $\mathrm{SiC}$ and OPyC layers that is thought to be due to the fact that this interface is weaker than the OPyC/matrix interface, which results in the OPyC being pulled away from the $\mathrm{SiC}$ as the matrix material contracts. This gap was observed in the pre-safety test PIE of particles from Compact 4-4-2 [Hunn et al. 2013a, 35] and has been observed to widen as a function of increasing safety test temperature [Hunn et al. 2013b, 27]. After other $1800^{\circ} \mathrm{C}$ safety tests, some particles with failed $\mathrm{SiC}$ experienced $\mathrm{OPyC}$ failure during deconsolidation and pre-burn leaching that resulted in kernel leaching [Hunn et al. 2014e, 8]. It has also been observed when particles are inspected in preparation for IMGA survey that the number of particles that are missing their OPyC layer after particle deconsolidation tends to increase with increasing safety test temperature.

It is a matter for debate whether fracture of the $\mathrm{SiC}$ in the Compact 4-4-2 failed-TRISO particles occurred first, precipitating the observed fission product redistribution and severe $\mathrm{SiC}$ degradation, or the $\mathrm{SiC}$ degradation led to the observed $\mathrm{SiC}$ fracture. It is likely that both processes were involved in generating the final observed microstructure. Examination of the Compact 4-4-2 failed-TRISO particles with x-ray tomography and materialographic cross sectioning revealed evidence of the same kind of radiationinduced damage that was observed to lead to SiC failure in AGR-1 particles during irradiation and postirradiation safety testing [Hunn et al. 2014b]. For instance, Figure $12 \mathrm{f}$ shows an example of the spearhead-like fracture that can occur when a crack in the buffer propagates directly into the adjacent IPyC layer. The other common mode for cracking the IPyC can be seen in Figure 16b, where stress at a buffer/IPyC delamination boundary apparently caused a tear in the IPyC layer. Both of these IPyC cracks, which most likely occurred during the irradiation test as a result of radiation-induced shrinkage in the buffer, were clearly associated with the $\mathrm{SiC}$ damage in these two failed-TRISO particles, so radiationinduced densification of the buffer coupled with IPyC cracking still appears to be a precursor to the 
observed failure during heating. It is possible that the $\mathrm{SiC}$ layer in the failed-TRISO particles was weakened by localized palladium and uranium degradation near an IPyC crack, making these particles more susceptible to $\mathrm{SiC}$ fracture. However, the $\mathrm{SiC}$ fracture (coupled with failure of all three gas-retentive layers) clearly resulted in additional degradation that would not have occurred otherwise. Evidence for the cracked $\mathrm{SiC}$ leading to enhanced degradation can be seen in Figure 14, where $\mathrm{SiC}$ degradation appeared to radiate out from the cracks shown in these micrographs.

Four of the Compact 4-4-2 particles with failed TRISO exhibited excessive uranium dispersion and fission product release that even resulted in visible loss of kernel mass. This was likely due to the gasretention failure changing the chemical balance in the kernel, with the free release of CO favoring carbon reduction of the uranium oxide at $1800^{\circ} \mathrm{C}$. This observed behavior illustrates the greater impact of full TRISO failure compared to $\mathrm{SiC}$ failure when at least one of the gas-tight pyrolytic carbon layers remains intact. AGR-1 particles with failed SiC but intact OPyC layers did not exhibit this kernel instability or the associated high levels of fission product release.

Previous $1800^{\circ} \mathrm{C}$ safety tests of AGR-1 Capsule 4 compacts have shown an increased release rate for silver, europium, and strontium after roughly $70-150 \mathrm{~h}$ at $1800^{\circ} \mathrm{C}$ [Morris et al. 2014]. This indicated the onset of measurable diffusive release through the $\mathrm{SiC}$ during the safety test, as opposed to the release observed in the first half of the $1800^{\circ} \mathrm{C}$ tests and during 300 -hour testing at lower temperature that could be explained by release from the matrix graphite of material released through the $\mathrm{SiC}$ during the 3-year irradiation. By extending the $1800^{\circ} \mathrm{C}$ heating period to over $650 \mathrm{~h}$ for the Compact 4-4-2 loose particle test, this diffusive release has been confirmed.

In previous compact safety tests, silver release at the start of the test was typically high and dropped off to a much smaller rate after a short time at the test temperature; this has been explained as quick depletion of silver trapped in the compact matrix. In the loose particle test, initial release of ${ }^{110 \mathrm{~m}} \mathrm{Ag}$ was low because the compact matrix had been removed. The release rate of ${ }^{110 \mathrm{~m}} \mathrm{Ag}$ was observed to increase noticeably after $\sim 150 \mathrm{~h}$, similar to previous $1800^{\circ} \mathrm{C}$ tests of Capsule 4 compacts, settling into a nearly linear release rate for about $200 \mathrm{~h}$ before dropping off due to depletion of the silver in each particle. IMGA measurements provided irrefutable evidence that silver was released from the particles at $1800^{\circ} \mathrm{C}$, with ${ }^{110 \mathrm{~m}} \mathrm{Ag}$ inventories down below the detection level for almost every particle by the end of the test.

Europium and strontium showed a slight decrease in the release rate over the first $150 \mathrm{~h}$ followed by a slightly higher and near constant release rate over the rest of the test. Initial release was probably dominated by slow europium and strontium release from the three particles whose TRISO layers failed during the ramp up to $1800^{\circ} \mathrm{C}$, while the later constant-rate release indicates general diffusive release through intact $\mathrm{SiC}$. This conclusion is strongly supported for europium, where the the total furnace release was more than three times the inventory that would have come from the failed-TRISO particles and measurable release from many of the particles with intact SiC was confirmed with the IMGA. Strontium release was lower, and could be explained by release from the five particles with failed TRISO. Beta emission from ${ }^{90} \mathrm{Sr}$ could not be measured with the IMGA to confirm release from individual particles.

Minor concentrations of palladium were observed predominantly at the IPyC/SiC interface of the crosssectioned particles that did not have failed TRISO, but clusters of palladium were nearly-absent in the $\mathrm{SiC}$ layers of all the particles or anywhere in the particles with failed TRISO. This conspicuous absence of palladium in the $\mathrm{SiC}$ has previously been observed after $1800^{\circ} \mathrm{C}$ safety testing and is understood to indicate that palladium silicide formed during irradiation migrates out of the $\mathrm{SiC}$ at this temperature [Hunn et al. 2013b]. Evidence for this out-diffusion of palladium from the SiC layer was also observed in the Compact 4-4-2 loose particles after the $1800^{\circ} \mathrm{C}$ test. Dark spots that were determined to be C-rich and Si-poor were observed in many of the $\mathrm{SiC}$ cross sections (e.g., Figure 36) and $\mathrm{Si}$ deposits were found elsewhere in the particle. Previous AGR-1 PIE has indicated that palladium played a major role in the observed degradation of the $\mathrm{SiC}$ [Hunn et al. 2014b], but in this loose particle study, it appears that 
uranium may have had more of a role in much of the observed degradation. Many of the heavily-degraded sites were surrounded by large deposits of uranium (e.g., Figure 25 and Figure 30).

In addition to degradation of the $\mathrm{SiC}$ via reaction with uranium, palladium, and possibly other fission products, general degradation at the $\mathrm{SiC} / \mathrm{OPyC}$ interface, observed in almost every analyzed cross section, suggests that thermal decomposition may also have occurred over the 650 -hour heating period at $1800^{\circ} \mathrm{C}$, as discussed in conjunction with Figure 34. Previous shorter $1800^{\circ} \mathrm{C}$ safety tests have not shown this behavior, which was apparently dependent on the longer heating time or absence of surrounding matrix.

In general, the loose particle heating test was very effective at resolving details about the fission product release from individual particles, both as a result of layer failure and diffusion. The ability to heat for longer time periods and perform IMGA measurements before and after the heating test were two major benefits of this type of experiment. Additional capabilities are currently being developed to take advantage of these two aspects. A small dedicated furnace will be used to heat small numbers of loose particles without the added complications performing the experiment within a shielded hot cell. This will allow for the longer heating times needed to produce measurable diffusive release through the $\mathrm{SiC}$, especially at lower test temperatures. In addition, the more complicated equipment and operation surrounding the capture of time-dependent release on deposition cups can be replaced by IMGA measurements and multiple tests with various test temperatures and durations, perhaps using multiple low-cost furnaces to increase experimental throughput. 


\section{REFERENCES}

Baldwin, C.A., J.D. Hunn, R.N. Morris, F.C. Montgomery, C.M. Silva, and P.A. Demkowicz. 2012. "First Elevated Temperature Performance Testing of Coated Particle Fuel Compacts from the AGR-1 Irradiation Experiment." Paper HTR2012-3-027 in Proceedings of the 6th International Topical Meeting on High Temperature Reactor Technology-HTR 2012, Tokyo, Japan, October 28November 1, 2012. Also published in Nuclear Engineering and Design 271: 131-141.

Demkowicz, P.A., L.T. Cole, S.A. Ploger, and P.L. Winston. 2011. AGR-1 Irradiation Test Train Preliminary Inspection and Disassembly First Look. INL/EXT-10-20722, Revision 0. Idaho Falls, Idaho: Idaho National Laboratory.

Demkowicz, P.A. 2014. Irradiated AGR-1 Compact 4-4-2 Loose-Particle Heating Test Examination Plan. PLN-4650, INL/MIS-14-31107. Idaho Falls, Idaho: Idaho National Laboratory.

Demkowicz, P.A., J.D. Hunn, S.A. Ploger, R.N. Morris, C.A. Baldwin, J.M. Harp, P.L. Winston, T.J. Gerczak, I.J. van Rooyen, F.C. Montgomery, and C.M. Silva. 2014. "Irradiation Performance of AGR-1 High Temperature Reactor Fuel." Paper HTR2014-31182 in Proceedings of the 7th International Topical Meeting on High Temperature Reactor Technology—HTR 2014, Weihai, China, October 27-31, 2014. Also to be published in Nuclear Engineering and Design 2015.

Demkowicz, P.A., E.L. Reber, D.M. Scates. L. Scott, B.P. Collin. 2015a. "First High Temperature Safety Tests of AGR-1 TRISO Fuel with the Fuel Accident Condition Simulator (FACS) Furnace." Journal of Nuclear Materials 464: 320-330.

Demkowicz, P.A., J.D. Hunn, R.N. Morris, I.J. van Rooyen, T.J. Gerczak, J.M. Harp, S.A. Ploger. $2015 b$. AGR-1 Post Irradiation Examination Final Report. INL/EXT-15-36407. Idaho Falls, Idaho: Idaho National Laboratory.

Harp, J.M. 2013. Analysis of Individual Compact Fission Product Inventory and Burnup of the AGR-1 TRISO Experiment Using Gamma Spectrometry. INL/ECAR-1682, Revision 2. Idaho Falls, Idaho: Idaho National Laboratory.

Hawkes, G.L. 2014. AGR-2 Daily As-Run Thermal Analyses. INL/ECAR-2476, Revision 1. Idaho Falls, Idaho: Idaho National Laboratory.

Hunn, J.D., and R.A. Lowden. 2006. Data Compilation for AGR-1 Variant 3 Coated Particle Composite LEU01-49T. ORNL/TM-2006/022, Revision 0. Oak Ridge, Tennessee: Oak Ridge National Laboratory.

Hunn, J.D., F.C. Montgomery, and P.J. Pappano. 2006. Data Compilation for AGR-1 Variant 3 Compact Lot LEU01-49T-Z. ORNL/TM-2006/510, Revision 0. Oak Ridge, Tennessee: Oak Ridge National Laboratory.

Hunn, J.D., T.W. Savage, and J.S. Kehn. 2012. AGR-1 Irradiated Compact 6-4-2 PIE Report: Preparation and Analysis of Polished Compact Sections. ORNL/TM-2012/285, Revision 0. Oak Ridge, Tennessee: Oak Ridge National Laboratory.

Hunn, J.D., T.W. Savage, and C.M. Silva. 2012. AGR-1 Fuel Compact Pre-Irradiation Characterization Summary Report. ORNL/TM-2012/295, Revision 0. Oak Ridge, Tennessee: Oak Ridge National Laboratory.

Hunn, J.D., R.N. Morris, C.A. Baldwin, F.C. Montgomery, C.M. Silva, and T.J. Gerczak. 2013a. AGR-1 Irradiated Compact 4-4-2 PIE Report: Evaluation of As-Irradiated Fuel Performance with Leach Burn Leach, IMGA, Materialography, and X-ray Tomography. ORNL/TM-2013/236, Revision 0. Oak Ridge, Tennessee: Oak Ridge National Laboratory. 
Hunn, J.D., R.N. Morris, C.A. Baldwin, F.C. Montgomery, C.M. Silva, and T.J. Gerczak. 2013b. PIE on Three Irradiated AGR-1 Compacts in FY2013. ORNL/LTR-2013/291, Revision 0. Oak Ridge, Tennessee: Oak Ridge National Laboratory.

Hunn, J.D., R.N. Morris, C.A. Baldwin, and F.C. Montgomery. 2014a. Safety-Test on Loose Particles from Irradiated Compact 4-4-2. ORNL/LTR-2014/486, Revision 0. Oak Ridge, Tennessee: Oak Ridge National Laboratory.

Hunn, J.D., C.A. Baldwin, T.J. Gerczak, F.C. Montgomery, R.N. Morris, C.M. Silva, P.A. Demkowicz, J.M. Harp, S.A. Ploger, I.J. van Rooyen, and K.E. Wright. 2014b. "Detection and Analysis of Particles with Breached SiC in AGR-1 Fuel Compacts." Paper HTR2014-31254 in Proceedings of the 7th International Topical Meeting on High Temperature Reactor Technology-HTR 2014, Weihai, China, October 27-31, 2014. Also to be published in Nuclear Engineering and Design 2015.

Hunn, J.D., R.N. Morris, C.A. Baldwin, and F.C. Montgomery. 2014c. Safety-Test on AGR-1 Compact 4-2-2. ORNL/LTR-2014/485, Revision 0. Oak Ridge, Tennessee: Oak Ridge National Laboratory.

Hunn, J.D., C.A. Baldwin, T.J. Gerczak, F.C. Montgomery, R.N. Morris, and C.M. Silva. 2014d. AGR-1 Irradiated Compacts 5-2-3 and 5-2-1 PIE Report: Evaluation of As-Irradiated Fuel Performance with Leach Burn Leach, IMGA, Materialography, and X-ray Tomography. ORNL/TM-2014/171, Revision 0. Oak Ridge, Tennessee: Oak Ridge National Laboratory.

Hunn, J.D., R.N. Morris, C.A. Baldwin, F.C. Montgomery, and T.J. Gerczak. 2014e. PIE on SafetyTested AGR-1 Compacts 5-3-3, 5-1-3, and 3-2-3. ORNL/TM-2014/484, Revision 0. Oak Ridge, Tennessee: Oak Ridge National Laboratory.

Hunn, J.D., R.N. Morris, C.A. Baldwin, F.C. Montgomery, and T.J. Gerczak. 2015. PIE on Safety-Tested AGR-1 Compact 4-2-2. ORNL/TM-2015/33, Revision 0. Oak Ridge, Tennessee: Oak Ridge National Laboratory.

Morris, R.N., C.A. Baldwin, P.A. Demkowicz, J.D. Hunn, and E.L. Reber. 2014. "Performance of AGR-1 High-Temperature Reactor Fuel During Post-Irradiation Heating Tests." Paper HTR2014-31135 in Proceedings of the 7th International Topical Meeting on High Temperature Reactor Technology, HTR 2014, Weihai, China, October 27-31, 2014. Also to be published in Nuclear Engineering and Design 2015.

Petti, D.A., J.T. Maki, J.D. Hunn, P.J. Pappano, C.M. Barnes, J.J. Saurwein, S.G. Nagley, J.M. Kendall, and R.R. Hobbins. 2010. "The DOE Advanced Gas Reactor Fuel Development and Qualification Program." JOM 62(9): 62-66.

Ploger, S.A., P.A. Demkowicz, J.D. Hunn, and J.S. Kehn. 2012. Ceramographic Examinations of Irradiated AGR-1 Fuel Compacts. INL/EXT-12-25301, Revision 1. Idaho Falls, Idaho: Idaho National Laboratory.

Sterbentz, J.W. 2013. JMOCUP As-Run Daily Depletion Calculation for the AGR-1 Experiment in ATR B-10 Position. INL/ECAR-958, Revision 2. Idaho Falls, Idaho: Idaho National Laboratory.

Wilhelm, H.A. 1964. The Carbon Reduction of Uranium Oxide. USAEC/IS-1023, Revision 0. Ames, Iowa: Ames Laboratory at Iowa State University of Science and Technology. 